Supporting Information for:

\title{
Asymmetric Carbon-Carbon Bond Formations in Conjugate Additions of Lithiated $N$-Boc Allylic and Benzylic Amines to Nitroalkenes: Enantioselective Synthesis of Substituted Piperidines, Pyrrolidines and Pyrimidinones
}

Timothy A. Johnson ${ }^{\dagger}$, Doo Ok Jang ${ }^{\ddagger}$, Brian W. Slafer ${ }^{\dagger}$, Michael D. Curtis ${ }^{\dagger}$ and Peter Beak ${ }^{\dagger *}$

tDepartment of Chemistry, Roger Adams Laboratory, University of Illinois at UrbanaChampaign, Urbana, Illinois 61801

‡Department of Chemistry, Yonsei University, Wonju 220-710, Korea

General. All lithiation reactions were performed in oven dried or flame dried glassware under a positive pressure of nitrogen with freshly distilled solvents. Tetrahydrofuran (THF) and diethyl ether was distilled from sodium and benzophenone. Toluene and dichloromethane were distilled from $\mathrm{CaH}_{2}$. (-)-Sparteine was distilled from the commercially available (Aldrich) compound and stored under nitrogen. Commercial tert-BuLi (solution in pentane), sec-BuLi (solution in cyclohexane), and $n$-BuLi (solution in hexanes) were titrated prior to use against $N$ pivaloyl-o-toluidine according to literature procedure. ${ }^{1}$ Nitroalkenes were either obtained from Aldrich or synthesized according to literature procedure. ${ }^{2}$

Column chromatography was performed on silica gel (230-400 mesh). Preparative high pressure liquid chromatography (HPLC) was performed using Waters Delta 600 pump system equipped with Nova-Pak@6fJ.m silica column (Waters, 19 x 300 mm). Analytical HPLC was performed using Rainin HPXL pump systems. Melting points (mp) were obtained using a Thomas-Hoover melting point apparatus and are uncorrected. The University of Illinois George L. Clark X-ray Facility performed all X-ray crystallography. The University of Illinois Mass Spectrometry Center performed all Mass spectrometry. The ZAB-SE mass spectrometer was purchased in part with grants from the Division of Research Resources, National Institutes of Health (RR 01575), the National Science Foundation (PCM 8121494), and the National Institute of General Medical Sciences (GM 27029). The 70-VSE mass spectrometer was purchased in part with a grant from the Division of Research Resources, National Institutes of Health (RR 04648). The University of Illinois Microanalytical Service Laboratory performed all elemental analysis. 
When an elemental analysis was not obtained, purity was established based on ${ }^{1} \mathrm{H} \mathrm{NMR},{ }^{13} \mathrm{C}$ NMR spectra and HRMS. When CSP-HPLC was used for enantiomeric purity analyses, authentic racemic materials for determing enantiomeric excess were prepared under the same reaction conditions for the chiral compounds except using achiral dibutylbispidine ${ }^{3}$ instead of ()-sparteine. Analytical chiral stationary phase (CSP) HPLC was performed on either Whelk-O (Regis Chemical Co., 25 cm x 4.6 mm i.d.) or Chiral-Pak Ad chiral column (Chiral Technologies INC, $25 \mathrm{~cm}$ x $4.6 \mathrm{~mm}$ i.d.) using mixtures of isopropanol (IPA) and hexane. Diastereomeric ratios were determined by ${ }^{1} \mathrm{H}$ NMR integration.

Allyl-(4-methoxy-phenyl)-carbamic Acid tert-Butyl Ester (5). To a stirred solution of allyl bromide $(1.07 \mathrm{~mL}, 12.3 \mathrm{mmol})$ in THF $(30 \mathrm{~mL})$ was added $\mathrm{NaH}(0.377 \mathrm{~g}, 15.7 \mathrm{mmol})$. The reaction mixture was cooled to $0{ }^{\circ} \mathrm{C}$ and a solution of $N$-Boc anisidine $(2.50 \mathrm{~g}, 11.20 \mathrm{mmol})$ in THF (20 mL) was added dropwise. After complete addition, the reaction was warmed to, stirred overnight, and carefully quenched with $\mathrm{H}_{2} \mathrm{O}(40 \mathrm{~mL})$. The aqueous was extracted with ether $(3 \mathrm{x}$ $50 \mathrm{~mL}$ ) and the combined organics were dried over $\mathrm{MgSO}_{4}$ and concentrated. Purification by silica gel column chromatography (8:1 hexanes/EtOAc) provided 5 (2.82 g, $96 \%)$ as an oil. ${ }^{\mathbf{1}} \mathbf{H}$ NMR $\left(\mathrm{CDCl}_{3}, 500 \mathrm{MHz}\right) \delta 1.45\left(\mathrm{~s}, 9 \mathrm{H},\left(\mathrm{CH}_{3}\right)_{3} \mathrm{CO}\right), 3.79\left(\mathrm{~s}, 3 \mathrm{H}, \mathrm{C}_{3} \mathrm{O}\right), 4.18(\mathrm{~d}, J=5.7 \mathrm{~Hz}, 1 \mathrm{H}$,

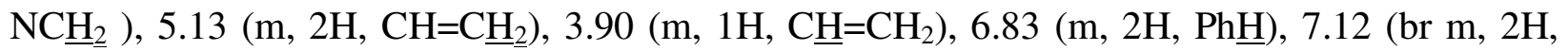
$\mathrm{Ph} \underline{\mathrm{H}}) ;{ }^{13} \mathrm{C}$ NMR $\left(\mathrm{CDCl}_{3}, 125.6 \mathrm{MHz}\right) \delta 28.2\left(\mathrm{CH}_{3}\right), 53.0\left(\mathrm{CH}_{2}\right), 55.3\left(\mathrm{CH}_{3}\right), 78.0(\mathrm{C}), 113.7$ $(\mathrm{CH}), 116.6\left(\mathrm{CH}_{2}\right), 127.8(\mathrm{CH}), 134.3(\mathrm{CH}), 135.6(\mathrm{C}), 154.7(\mathrm{C}), 157.4(\mathrm{C})$. Anal. Calcd for $\mathrm{C}_{15} \mathrm{H}_{21} \mathrm{NO}_{3}$ : C, 68.42; H, 8.04; N, 5.32. Found C, 68.25; H, 8.04; N, 5.45.

\section{(4-Methoxy-phenyl)-(5-nitro-3-phenyl-pent-1-enyl)-carbamic Acid tert-Butyl}

Ester (23). The general lithiation procedure was followed using 2 (3.0 g, $8.84 \mathrm{mmol})$ and nitroethylene $^{2 \mathrm{~b}} 16$ (0.645 g, $\left.8.84 \mathrm{mmol}\right)$ premixed with $\mathrm{TMSCl}(1.12 \mathrm{~mL}, 8.84 \mathrm{mmol})$. Purification by column chromatography (4:1 pet ether/EtOAc) gave 23 (3.06 g, $82 \%)$ as a colorless oil. ${ }^{1} \mathbf{H}$ NMR (acetone- $\left.d_{6}, 500 \mathrm{MHz}\right) \delta 1.42(\mathrm{~s}, 9 \mathrm{H}, \mathrm{O} t-\mathrm{Bu}), 2.12(\mathrm{~m}, 1 \mathrm{H}$, $\left.\mathrm{NO}_{2} \mathrm{CH}_{2} \underline{\mathrm{CH}}_{2}\right), 2.18\left(\mathrm{~m}, 1 \mathrm{H}, \mathrm{NO}_{2} \mathrm{CH}_{2} \underline{\mathrm{CH}}_{2}\right), 3.11$ (m, 1H, $\left.\underline{\mathrm{HPh}}\right), 3.82(\mathrm{~s}, 3 \mathrm{H}, \mathrm{OMe}), 4.26$ $\left(\mathrm{m}, 2 \mathrm{H}, \mathrm{CH}_{2} \mathrm{NO}_{2}\right), 5.06(\mathrm{t}, J=9.7 \mathrm{~Hz}, 1 \mathrm{H}, \mathrm{CH}=\mathrm{CHN}), 6.70(\mathrm{~d}, J=9.0 \mathrm{~Hz}, 1 \mathrm{H}, \mathrm{CH}=\mathrm{CHN})$, $6.88(\mathrm{~m}, 4 \mathrm{H}, \mathrm{Ph} \underline{\mathrm{H}}), 7.11-7.22(\mathrm{~m}, 5 \mathrm{H}, \mathrm{PhH}) ;{ }^{13} \mathbf{C}$ NMR (acetone- $\left.d_{6}, 125.6 \mathrm{MHz}\right) \delta 28.0$ $\left(\mathrm{CH}_{3}\right), 34.5\left(\mathrm{CH}_{2}\right), 39.4(\mathrm{CH}), 55.6\left(\mathrm{CH}_{3}\right), 74.0\left(\mathrm{CH}_{2}\right), 81.1(\mathrm{C}), 114.6(\mathrm{CH}), 127.1(\mathrm{CH})$, 
$127.7(\mathrm{CH}), 128.9(\mathrm{CH}), 129.0(\mathrm{CH}), 129.4(\mathrm{CH}), 135.5(\mathrm{C}), 143.3(\mathrm{C}), 153.7(\mathrm{C}), 158.8$ (C). Anal. Calcd for $\mathrm{C}_{23} \mathrm{H}_{28} \mathrm{~N}_{2} \mathrm{O}_{5}$ : C, 66.97; H, 6.84; N, 6.79. Found C, 66.85; H, 6.94; N, 6.70. $[\alpha]_{\mathrm{D}}^{20}:+175.1^{\circ}(c=1.4, \mathrm{MeOH})$.

(3,6-Dimethyl-4-nitromethyl-hept-1-enyl)-(4-methoxy-phenyl)-carbamic Acid tert-Butyl Ester (26). The general lithiation procedure was followed using 4 ( $0.256 \mathrm{~g}, 0.922$ $\mathrm{mmol})$ and 12 (0.155 g, $1.19 \mathrm{mmol})$. Purification by column chromatography (10:1 pet ether/EtOAc) gave $\mathbf{2 6}(0.277 \mathrm{~g}, 74 \%, 84: 16 \mathrm{dr})$ as a colorless oil. Major diastereomer: ${ }^{1} \mathbf{H}$ NMR (acetone- $\left.d_{6}, 500 \mathrm{MHz}\right) \delta 0.61\left(\mathrm{~d}, J=6.6 \mathrm{~Hz}, 3 \mathrm{H}, \mathrm{CHCH}_{3}\right), 0.73(\mathrm{~d}, J=6.6 \mathrm{~Hz}$, $3 \mathrm{H}, \mathrm{CHC}_{3}$ ), $0.79\left(\mathrm{~d}, J=6.6 \mathrm{~Hz}, 3 \mathrm{H}, \mathrm{CHC}_{3}\right.$ ), 0.94 (quint, $J=6.9 \mathrm{~Hz}, 1 \mathrm{H}, \mathrm{CHC}_{2} \mathrm{CH}$ ), 1.15 (quint, $J=6.9 \mathrm{~Hz}, 1 \mathrm{H}, \mathrm{CHCH}_{2} \mathrm{CH}$ ), 1.36 (s, 9H, Ot-Bu), 1.44 (nonet, $J=6.6 \mathrm{~Hz}, 1 \mathrm{H}$,

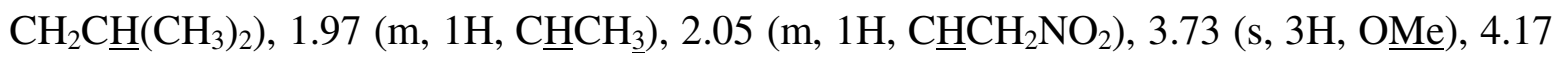
$\left(\mathrm{dd}, J=12.6,7.9 \mathrm{~Hz}, 1 \mathrm{H}, \mathrm{C}_{2} \mathrm{NO}_{2}\right), 4.36\left(\mathrm{dd}, J=12.4,5.3 \mathrm{~Hz}, 1 \mathrm{H}, \mathrm{C}_{2} \mathrm{NO}_{2}\right), 4.64(\mathrm{t}, J=$ $9.0 \mathrm{~Hz}, 1 \mathrm{H}, \mathrm{C} \underline{H}=\mathrm{CHN}), 6.52(\mathrm{~d}, J=8.9 \mathrm{~Hz}, 1 \mathrm{H}, \mathrm{CH}=\mathrm{C} \underline{H N}), 6.85(\mathrm{~m}, 2 \mathrm{H}, \mathrm{Ph} \underline{\mathrm{H}}), 7.13(\mathrm{~m}$, $2 \mathrm{H}, \mathrm{Ph} \underline{\mathrm{H}}) ;{ }^{13} \mathrm{C}$ NMR (acetone- $\left.d_{6}, 125.6 \mathrm{MHz}\right) \delta 16.8\left(\mathrm{CH}_{3}\right), 22.7\left(\mathrm{CH}_{3}\right), 23.0\left(\mathrm{CH}_{3}\right), 25.8$ $(\mathrm{CH}), 28.3\left(\mathrm{CH}_{3}\right), 32.4(\mathrm{CH}), 39.4\left(\mathrm{CH}_{2}\right), 41.1(\mathrm{CH}), 55.6\left(\mathrm{CH}_{3}\right), 78.6\left(\mathrm{CH}_{2}\right), 81.1(\mathrm{C})$, $114.5(\mathrm{CH}), 128.6(\mathrm{CH}), 130.0(\mathrm{CH}), 136.0(\mathrm{C}), 153.9(\mathrm{C}), 158.6(\mathrm{C})$. Anal. Calcd for $\mathrm{C}_{22} \mathrm{H}_{34} \mathrm{~N}_{2} \mathrm{O}_{5}:$ C, 65.00; H, 8.43; N, 6.89. Found C, 64.95; H, 8.44; N, 6.98 .

(4-Methoxy-phenyl)-(5-nitro-4-phenyl-pent-1-enyl)-carbamic Acid tert-Butyl Ester (27). The general lithiation procedure was followed using 5 (3.37 g, $12.8 \mathrm{mmol})$ and $10(2.67 \mathrm{~g}, 17.89 \mathrm{mmol})$. Purification by column chromatography (4:1 pet ether/EtOAc) gave 27 (3.92 g, $74 \%$ ) as a colorless oil. ${ }^{1} \mathbf{H}$ NMR (acetone- $\left.d_{6}, 500 \mathrm{MHz}\right) \delta 1.41(\mathrm{~s}, 9 \mathrm{H}, \mathrm{O} t$ $\underline{\mathrm{Bu}}), 1.97\left(\mathrm{~m}, 2 \mathrm{H}, \underline{\mathrm{C}}_{2} \mathrm{CH}=\mathrm{C} \underline{\mathrm{H}}\right), 3.30$ (quint, $\left.J=6.6 \mathrm{~Hz}, 1 \mathrm{H}, \mathrm{C} \underline{\mathrm{HPh}}\right), 3.81$ (s, 3H, $\underline{\mathrm{OMe}}$ ), $4.59\left(\mathrm{~m}, 2 \mathrm{H}, \mathrm{C}_{2} \mathrm{NO}_{2}\right), 4.72(\mathrm{q}, J=8.1 \mathrm{~Hz}, 1 \mathrm{H}, \mathrm{C} \underline{\mathrm{H}}=\mathrm{CHN}), 6.52(\mathrm{~d}, J=9.0 \mathrm{~Hz}, 1 \mathrm{H}$,

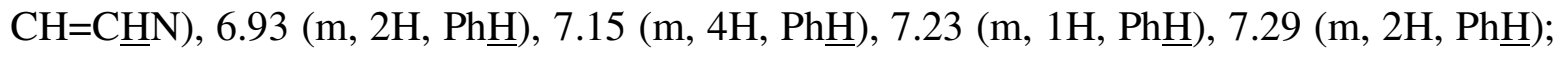
${ }^{13} \mathbf{C}$ NMR (acetone- $\left.d_{6}, 125.6 \mathrm{MHz}\right) \delta 28.1\left(\mathrm{CH}_{3}\right), 30.8\left(\mathrm{CH}_{2}\right), 44.5(\mathrm{CH}), 55.6\left(\mathrm{CH}_{3}\right), 80.3$ $\left(\mathrm{CH}_{2}\right), 81.0(\mathrm{C}), 114.6(\mathrm{CH}), 128.0(\mathrm{CH}), 128.3(\mathrm{CH}), 129.1(\mathrm{CH}), 129.2(\mathrm{CH}), 130.4(\mathrm{CH})$, 135.4 (C), 140.7 (C), 153.6 (C), 158.7 (C). Anal. Calcd for $\mathrm{C}_{23} \mathrm{H}_{28} \mathrm{~N}_{2} \mathrm{O}_{5}$ : C, 66.97; H, 6.84; N, 6.79. Found C, 66.86; H, 6.81; N, 6.74. 
(4-Methoxy-phenyl)-(3-nitro-l,2-diphenyl-propyl)-carbamic Acid tert-Butyl Ester (37). The general lithiation procedure was followed using $28(0.100 \mathrm{~g}, 0.32 \mathrm{mmol})$ and $\mathbf{3 0}(0.070$ g, $0.48 \mathrm{mmol})$. Purification by column chromatography (9:1 hexanes/EtOAc) gave the 37 (0.120 $\mathrm{g}, 81 \%, 68: 32 \mathrm{dr}$ ). The diastereomers were separated by recrystallization from $\mathrm{CH}_{2} \mathrm{Cl}_{2} / \mathrm{EtOH}$. Major diastereomer: mp 140-142 ${ }^{\circ} \mathrm{C} ;{ }^{1} \mathbf{H}$ NMR $\left(500 \mathrm{MHz}, \mathrm{CDCl}_{3}\right) \delta 1.36(\mathrm{~s}, 9 \mathrm{H}), 3.81(\mathrm{~s}, 3 \mathrm{H})$, $4.24(\mathrm{~s}, 1 \mathrm{H}), 4.97(\mathrm{t}, J=11.0 \mathrm{~Hz}, 1 \mathrm{H}), 6.00(\mathrm{~s}, 1 \mathrm{H}), 6.76(\mathrm{~d}, J=6.5 \mathrm{~Hz}, 2 \mathrm{H}), 6.86(\mathrm{~d}, J=6.5 \mathrm{~Hz}$, 2H), 6.98-7.22 (m, 8H); ${ }^{13} \mathbf{C}$ NMR (125.7 MHz, $\left.\mathrm{CDCl}_{3}\right) \delta$ 28.2, 46.6, 55.2, 61.8, 80.3, 80.7, 113.6, 127.5, 127.6, 127.7, 128.2, 128,7, 129.6, 130.8, 136.3, 137.0, 156.1, 158.5. Anal. Calcd for $\mathrm{C}_{27} \mathrm{H}_{30} \mathrm{~N}_{2} \mathrm{O}_{5}$ : C, 70.11; H, 6.54; N, 6.06. Found: C, 70.02; H, 6.52; N, 6.12. The enantiomeric ratio of the major diastereomer was determined by CSP-HPLC on a Whelk-O column with $2.5 \%$ IPA/hexanes mobile phase and a flow rate of $1.0 \mathrm{~mL} / \mathrm{min}: \mathrm{rt}=12.3 \mathrm{~min}(>99 \%), 13.4 \mathrm{~min}<1$ \%). Minor diastereomer: ${ }^{1} \mathbf{H}$ NMR $\left(400 \mathrm{MHz}, \mathrm{CDCl}_{3}\right) \delta 1.18$ (s, 9H), 3.77 (s, 3H), 4.38 (s, $3 \mathrm{H}), 5.77(\mathrm{~s}, 1 \mathrm{H}), 6.16(\mathrm{~s}, 2 \mathrm{H}), 6.64(\mathrm{~d}, J=6.4 \mathrm{~Hz}, 2 \mathrm{H}), 7.34-7.54(\mathrm{~m}, 10 \mathrm{H}) ;{ }^{13} \mathbf{C ~ N M R}(125.7$ $\left.\mathrm{MHz}, \mathrm{CDCl}_{3}\right) \delta 28.1,45.5,55.2,61.9,80.0,113.2,128.1,128.6,128.7,128.8,128.9,129.0$, 137.2, 137.7, 155.1, 158.3. HRMS Calcd for $\mathrm{C}_{27} \mathrm{H}_{30} \mathrm{~N}_{2} \mathrm{O}_{5}$ : 462.2155; Found: 462.2154.

(4-Methoxy-phenyl)-(3-methyl-2-nitromethyl-l-phenyl-butyl)-carbamic Acid tertButyl Ester (38). The general lithiation procedure was followed using 28 (0.5 g, $1.60 \mathrm{mmol})$ and 31 (0.086 g, $0.75 \mathrm{mmol})$. Purification by column chromatography (95:5 hexanes/EtOAc) gave 38 (0.190 g, 90\%, >99:1 dr). Major diastereomer: mp 106-106 ${ }^{\circ} \mathrm{C} ;{ }^{1} \mathbf{H}$ NMR $\left(400 \mathrm{MHz}, \mathrm{CDCl}_{3}\right) \delta$ $0.80(\mathrm{~d}, J=6.8 \mathrm{~Hz}, 3 \mathrm{H}), 0.87$ (d, J= 7.2 Hz, 3H), 1.31 (s, 9H), 1.60-1.70 (m, 1H), 3.19 (s, 1H), $3.78(\mathrm{~s}, 3 \mathrm{H}), 4.68-4.86(\mathrm{~m}, 2 \mathrm{H}), 5.45(\mathrm{~s}, 1 \mathrm{H}), 6.55(\mathrm{~s}, 2 \mathrm{H}), 6.70(\mathrm{~d}, J=8.0 \mathrm{~Hz}, 2 \mathrm{H}), 6.94(\mathrm{~d}, J=$ 7.2 Hz, 2H), 7.14-7.27 (m, 3H); ${ }^{13} \mathbf{C}$ NMR (100 MHz, $\left.\mathrm{CDCl}_{3}\right) \delta 15.3,20.9,27.5,28.4,44.4$, 55.5, 61.9, 74.1, 80.7, 113.6, 128.1, 128.3, 129.6, 131.2, 136.9, 156.4, 158.6. Anal. Calcd for $\mathrm{C}_{24} \mathrm{H}_{32} \mathrm{~N}_{2} \mathrm{O}_{5}$ : C, 67.27; H, 7.53; N, 6.54. Found: C, 67.23; H, 7.58; N, 6.58. The enantiomeric ratio of the major diastereomer was determined by CSP-HPLC on a Chiral Pak-AD column with $2 \%$ IPA/hexanes mobile phase and a flow rate of $0.4 \mathrm{~mL} / \mathrm{min}: \mathrm{rt}=19.5 \mathrm{~min}(3 \%), 20.7 \mathrm{~min}(97$ $\%)$. 
(4-Methoxy-phenyl)-(2-methyl-3-nitro-l-phenyl-propyl)-carbamic Acid tert-Butyl

Ester (39). The general lithiation procedure was followed using 28 (0.310 g, $1.0 \mathrm{mmol})$ and 32 (0.13 g, $1.5 \mathrm{mmol})$. Purification by column chromatography (9:1 hexanes/EtOAc) gave 39 (0.38 g, 95\%, 90:10 dr). The diastereomers were separated by flash column chromatography. Suitable crystals for X-ray diffraction of the title compound were obtained by slow evaporation from $\mathrm{Et}_{2} \mathrm{O}$ /pet ether. Major diastereomer: mp $125-126{ }^{\circ} \mathrm{C} ;{ }^{1} \mathbf{H} \mathbf{N M R}\left(500 \mathrm{MHz}, \mathrm{CDCl}_{3}\right) \delta 0.86(\mathrm{~d}, J$ $=6.5 \mathrm{~Hz}, 3 \mathrm{H}), 1.33(\mathrm{~s}, 9 \mathrm{H}), 3.76(\mathrm{~s}, 3 \mathrm{H}), 4.52(\mathrm{t}, J=11.0 \mathrm{~Hz}, 1 \mathrm{H}), 4.97(\mathrm{~d}, J=11.5 \mathrm{~Hz}, 1 \mathrm{H})$, $5.26(\mathrm{~s}, 1 \mathrm{H}), 6.58(\mathrm{~s}, 2 \mathrm{H}), 6.71(\mathrm{~d}, J=8.5 \mathrm{~Hz}, 2 \mathrm{H}), 6.94(\mathrm{~d}, J=6.5 \mathrm{~Hz}, 2 \mathrm{H}), 7.14-7.28(\mathrm{~m}, 3 \mathrm{H})$; ${ }^{13} \mathrm{C}$ NMR $\left(125.7 \mathrm{MHz}, \mathrm{CDCl}_{3}\right) \delta$ 15.7, 28.1, 34.9, 55.1, 63.3, 80.1, 80.3, 113.5, 127.9, 128.1, 129.2, 130.7, 131.0, 136.7, 155.6, 158.4, 158.5. Anal. Calcd for $\mathrm{C}_{22} \mathrm{H}_{28} \mathrm{~N}_{2} \mathrm{O}_{5}$ : C, 65.98; H, 7.05; $\mathrm{N}, 7.00$. Found: $\mathrm{C}, 65.63 ; \mathrm{H}, 7.00 ; \mathrm{N}, 7.00$. The enantiomeric ratio of the major diastereomer was determined by CSP-HPLC on a Whelk-O column with $1 \%$ IPA/hexanes mobile phase and a flow rate of $0.2 \mathrm{~mL} / \mathrm{min}: \mathrm{rt}=49.3 \mathrm{~min}(<1 \%), 51.3 \mathrm{~min}(>99 \%)$. Minor diastereomer: ${ }^{1} \mathbf{H}$ NMR $\left(500 \mathrm{MHz}, \mathrm{CDCl}_{3}\right) \delta 1.37(\mathrm{~s}, 9 \mathrm{H}), 1.44(\mathrm{~d}, J=6.0 \mathrm{~Hz}, 3 \mathrm{H}), 3.06-3.18(\mathrm{~m}, 1 \mathrm{H}), 3.80(\mathrm{~s}$, $3 \mathrm{H}), 4.00-4.07(\mathrm{~m}, 1 \mathrm{H}), 4.18(\mathrm{dd}, J=4.0,12.3 \mathrm{~Hz}, 1 \mathrm{H}), 5.27(\mathrm{~d}, J=11.5 \mathrm{~Hz}, 1 \mathrm{H}), 6.57(\mathrm{~d}, J=$ $7.0 \mathrm{~Hz}, 2 \mathrm{H}), 6.74(\mathrm{~d}, J=8.5 \mathrm{~Hz}, 2 \mathrm{H}), 7.03(\mathrm{~d}, J=7.0 \mathrm{~Hz}, 2 \mathrm{H}), 7.20-7.30(\mathrm{~m}, 3 \mathrm{H}) ;{ }^{13} \mathbf{C ~ N M R}$ $\left(125.7 \mathrm{MHz}, \mathrm{CDCl}_{3}\right) \delta 15.4,28.2,34.4,55.3,63.4,79.6,113.6,128.3,128.6,129.1,130.8$, 131.5, 137.0, 155.5, 158.5. HRMS Calcd for $\mathrm{C}_{22} \mathrm{H}_{28} \mathrm{~N}_{2} \mathrm{O}_{5}$ : 400.1998; Found: 400.1998.

(2-Cyclohexyl-3-nitro-l-phenyl-propyl)-(4-methoxy-phenyl)-carbamic Acid tertButyl Ester (40). The general 1ithiation procedure was followed using 28 (0.500 g, $1.60 \mathrm{mmo1})$ and 11 (0.372 g, $2.40 \mathrm{mmol})$. Purification by column chromatography (8.5:1.5 hexanes/EtOAc) gave $40(0.69 \mathrm{~g}, 92 \%, 92: 8 \mathrm{dr})$. The diastereomers were separated by preparative HPLC (9.7:0.3 hexanes/EtOAc). The major diastereomer was less retained. Major diastereomer: mp 144-146 ${ }^{\circ} \mathrm{C} ;{ }^{1} \mathbf{H}$ NMR $\left(400 \mathrm{MHz}, \mathrm{CDCl}_{3}\right) \delta$ 0.80-1.75 (m, 20H), 3.17 (s, 1H), 3.79 (s, 3H), 4.67-4.86 (m, 2H), $5.50(\mathrm{~s}, 1 \mathrm{H}), 6.56(\mathrm{~s}, 2 \mathrm{H}), 6.70(\mathrm{~d}, J=8.0 \mathrm{~Hz}, 2 \mathrm{H}), 6.93(\mathrm{~d}, J=6.8 \mathrm{~Hz}, 2 \mathrm{H}), 7.10-7.28(\mathrm{~m}$, $3 \mathrm{H}) ;{ }^{13} \mathrm{C}$ NMR $\left(125.7 \mathrm{MHz}, \mathrm{CDCl}_{3}\right) \delta$ 26.0, 26.1, 26.1, 26.6, 28.1, 31.1, 38.1, 44.1, 53.4, 55.2, 61.1, 74.7, 113.3, 127.9, 128.1, 129.3, 130.9, 136.7, 156.1, 158.3. Anal. Calcd for $\mathrm{C}_{27} \mathrm{H}_{36} \mathrm{~N}_{2} \mathrm{O}_{5}$ : C, 69.21; H, 7.74; N, 5.98. Found: C, 68.30; H, 7.70; N, 5.99. The enantiomeric ratio of the major diastereomer was determined by CSP-HPLC on a Chiral Pak AD column with $3 \%$ IPA/hexanes mobile phase and a flow rate of $0.3 \mathrm{~mL} / \mathrm{min}: \mathrm{rt}=13.0 \mathrm{~min}(96 \%), 14.6 \mathrm{~min}(4 \%)$. 
Minor diastereomer: ${ }^{1} \mathbf{H}$ NMR $\left(500 \mathrm{MHz}, \mathrm{CDCl}_{3}\right) \delta$ 1.00-2.30 (m, 20H), $3.08(\mathrm{~s}, 1 \mathrm{H}), 3.80(\mathrm{~s}$, 3H), $3.96(\mathrm{dd}, J=3.5,13.8 \mathrm{~Hz}, 1 \mathrm{H}), 4.33(\mathrm{dd}, J=7.5,14.0 \mathrm{~Hz}, 1 \mathrm{H}), 5.50(\mathrm{~s}, 1 \mathrm{H}), 6.56(\mathrm{~s}, 2 \mathrm{H})$, 6.70-7.30 (m, 7H); ${ }^{13} \mathrm{C}$ NMR (125.7 $\left.\mathrm{MHz}, \mathrm{CDCl}_{3}\right) \delta$ 26.4, 26.6, 26.7, 27.4, 28.2, 31.8, 37.7, 43.4, 55.3, 60.4, 74.5, 80.3, 113.6, 128.3, 128.5, 129.4, 130.9, 137.1, 155.4, 158.5. Anal. Calcd for $\mathrm{C}_{27} \mathrm{H}_{36} \mathrm{~N}_{2} \mathrm{O}_{5}$ : C, 69.21; H, 7.74; N, 5.98. Found: C, 68.26; H, 7.86; N, 5.92.

(4-Methoxy-phenyl)-(3-nitro-1-phenyl-2-triisopropylsilanyloxy-propyl)-carbamic Acid tert-Butyl Ester (41). The general lithiation procedure was followed using 28 (0.310 g, $1.00 \mathrm{mmol})$ and 15 (0.300 g, $1.50 \mathrm{mmol})$. Purification by column chromatography (9:1 hexanes/EtOAc) gave 41 (0.420 g, 82\%, 77:23 dr). The diastereomers were separated by preparative HPLC (93:7 hexanes/EtOAc). The major diastereomer was less retained. Major diastereomer: ${ }^{1} \mathbf{H}$ NMR $\left(500 \mathrm{MHz}, \mathrm{CDCl}_{3}\right) \delta$ 0.65-0.75 (m, 3H), $0.83(\mathrm{~d}, J=7.5 \mathrm{~Hz}, 9 \mathrm{H}), 0.90$ (d, $J=7.5 \mathrm{~Hz}, 9 \mathrm{H}), 1.36(\mathrm{~s}, 9 \mathrm{H}), 3.79(\mathrm{~s}, 3 \mathrm{H}), 4.73(\mathrm{~s}, 1 \mathrm{H}), 4.92(\mathrm{dd}, J=2.5,13.3 \mathrm{~Hz}, 1 \mathrm{H}), 5.04$ $(\mathrm{s}, 1 \mathrm{H}), 5.48-5.60(\mathrm{~m}, 1 \mathrm{H}), 6.75(\mathrm{~s}, 4 \mathrm{H}), 7.20-7.30(\mathrm{~m}, 5 \mathrm{H}) ;{ }^{13} \mathbf{C} \mathbf{~ N M R}\left(100 \mathrm{MHz}, \mathrm{CDCl}_{3}\right) \delta 12.9$, 18.0, 18.0, 28.2, 55.3, 67.2, 71.8, 113.7, 128.1, 128.1, 129.5, 129.9, 137.9, 158.3. HRMS FAB $(\mathrm{M}+1)$ Calcd for $\mathrm{C}_{30} \mathrm{H}_{46} \mathrm{~N}_{2} \mathrm{O}_{6} \mathrm{Si}$ : 559.3203; Found: 559.3204. The enantiomeric ratio of the major diastereomer was determined by CSP-HPLC on a Whelk-O column with $1 \%$ IPA/hexanes mobile phase and a flow rate of $0.2 \mathrm{~mL} / \mathrm{min}: \mathrm{rt}=46.2 \mathrm{~min}(88 \%), 48.7 \mathrm{~min}(12 \%)$. Minor diastereomer: ${ }^{1} \mathbf{H}$ NMR $\left(500 \mathrm{MHz}, \mathrm{CDCl}_{3}\right) \delta 1.04-1.16(\mathrm{~m}, 21 \mathrm{H}), 1.35(\mathrm{~s}, 9 \mathrm{H}), 3.81(\mathrm{~s}, 3 \mathrm{H})$, 4.34 (dd, $J=7.0,13.5 \mathrm{~Hz}, 1 \mathrm{H}), 4.50$ (d, $J=12.5 \mathrm{~Hz}, 1 \mathrm{H}), 5.23$ (d, $J=5.0 \mathrm{~Hz}, 1 \mathrm{H}), 5.45$ (s, 1H), $6.82(\mathrm{~d}, J=8.5 \mathrm{~Hz}, 2 \mathrm{H}), 7.03(\mathrm{~d}, J=8.5 \mathrm{~Hz}, 2 \mathrm{H}), 7.28-7.36(\mathrm{~m}, 3 \mathrm{H}), 7.38-7.44(\mathrm{~m}, 2 \mathrm{H}) ;{ }^{13} \mathbf{C}$ NMR $\left(125.7 \mathrm{MHz}, \mathrm{CDCl}_{3}\right) \delta 12.8,18.1,28.1,55.4,68.4,72.0,79.6,113.9,127.8,128.1,128.5$, 129.5, 137.0, 154.9, 158.2. HRMS Calcd for $\mathrm{C}_{30} \mathrm{H}_{46} \mathrm{~N}_{2} \mathrm{O}_{6} \mathrm{Si}$ : 558.3125; Found: 558.3125. The enantiomeric ratio of the minor diastereomer was determined by CSP-HPLC on a Whelk-O column with $1 \%$ IPA/hexanes mobile phase and a flow rate of $0.2 \mathrm{~mL} / \mathrm{min}: \mathrm{rt}=52.9 \mathrm{~min}(27$ $\%), 57.1 \min (73 \%)$.

(4-Methoxy-phenyl)-(3-nitro-l-phenyl-propyl)-carbamic Acid tert-Butyl Ester (42). The general lithiation procedure was followed using $28(0.310 \mathrm{~g}, 1.00 \mathrm{mmol})$ and $16(0.110 \mathrm{~g}$, $1.50 \mathrm{mmol})$. Purification by column chromatography (9:1 hexanes/EtOAc) gave 42 (0.270 g, 71 \%). ${ }^{1} \mathbf{H}$ NMR $\left(400 \mathrm{MHz}, \mathrm{CDCl}_{3}\right) \delta 1.40(\mathrm{~s}, 9 \mathrm{H}), 2.52-2.76(\mathrm{~m}, 2 \mathrm{H}), 3.78(\mathrm{~s}, 3 \mathrm{H}), 4.46-4.58(\mathrm{~m}$, 
2H), $5.59(\mathrm{t}, J=6.8 \mathrm{~Hz}, 1 \mathrm{H}), 6.59(\mathrm{~d}, J=8.4 \mathrm{~Hz}, 2 \mathrm{H}), 6.73(\mathrm{~d}, J=9.2 \mathrm{~Hz}, 2 \mathrm{H}), 7.10-7.18(\mathrm{~m}$, 2H), 7.24-7.34 (m, 3H); ${ }^{13} \mathbf{C}$ NMR $\left(100 \mathrm{MHz}, \mathrm{CDCl}_{3}\right) \delta 28.2,29.5,55.2,57.0,72.9,80.3,113.7$, $125.3,128.1,128.2,128.4,129.0,130.7,130.8,138.7,155.5,158.5$. HRMS Calcd for $\mathrm{C}_{21} \mathrm{H}_{26} \mathrm{~N}_{2} \mathrm{O}_{5}: 386.1842$; Found: 386.1842 . The enantiomeric ratio was determined by CSP-HPLC on a Chiral Pak AD column with $4 \% \mathrm{IPA} / \mathrm{hexanes}$ mobile phase and a flow rate of $0.5 \mathrm{~mL} / \mathrm{min}$ : $\mathrm{rt}=31.3 \min (4 \%), 36.0 \min (96 \%)$.

(2-Furan-2-yl-3-nitro-l-phenyl-propyl)-(4-methoxy-phenyl)-carbamic Acid tert-Butyl Ester (43). The general lithiation procedure was followed using 28 (0.300 g, $0.960 \mathrm{mmol})$ and 14 (0.200 g, $1.44 \mathrm{mmol})$. Purification by column chromatography (85:15 hexanes/EtOAc) gave the title compound $(0.610 \mathrm{~g}, 84 \%, 63: 37 \mathrm{dr})$. The diastereomers were separated by preparative HPLC (85:15 hexanes/EtOAc). The major diastereomer was less retained. Major diastereomer: ${ }^{1}$ H NMR $\left(500 \mathrm{MHz}, \mathrm{CDCl}_{3}\right) \delta 1.36(\mathrm{~s}, 9 \mathrm{H}), 3.80(\mathrm{~s}, 3 \mathrm{H}), 4.40-4.50(\mathrm{~m}, 1 \mathrm{H}), 4.96-5.14(\mathrm{~m}, 2 \mathrm{H})$, $5.86(\mathrm{~s}, 1 \mathrm{H}), 5.95(\mathrm{~d}, J=3.0 \mathrm{~Hz}, 1 \mathrm{H}), 6.03-6.10(\mathrm{~m}, 1 \mathrm{H}), 6.61(\mathrm{~s}, 2 \mathrm{H}), 6.76(\mathrm{~d}, J=8.0 \mathrm{~Hz}, 2 \mathrm{H})$, $6.94(\mathrm{~d}, J=7.5 \mathrm{~Hz}, 2 \mathrm{H}), 7.06-7.22(\mathrm{~m}, 4 \mathrm{H}) ;{ }^{13} \mathbf{C} \mathbf{N M R}\left(125.7 \mathrm{MHz}, \mathrm{CDCl}_{3}\right) \delta 28.2,40.9,55.3$, 61.0, 80.8, 109.3, 110.2, 113.7, 127.8, 129.0, 130.6, 136.6, 142.3, 149.7, 158.6. HRMS Calcd for $\mathrm{C}_{25} \mathrm{H}_{28} \mathrm{~N}_{2} \mathrm{O}_{6}$ : 452.1947; Found: 452.1947. The enantiomers of the major diastereomer were inseparable by CSP-HPLC. Minor diastereomer: ${ }^{1} \mathbf{H}$ NMR $\left(500 \mathrm{MHz}, \mathrm{CDCl}_{3}\right) \delta 1.28(\mathrm{~s}, 9 \mathrm{H})$, 3.79 (s, 3H), 4.27 (dd, $J=3.5,12.7 \mathrm{~Hz}, 1 \mathrm{H}), 4.50$ (dd, $J=10.5,12.8 \mathrm{~Hz}, 1 \mathrm{H}), 4.71(\mathrm{~s}, 1 \mathrm{H}), 5.53$ (s, 1H), 6.30-6.47 (m, 3H), $6.50(\mathrm{~d}, J=2.5 \mathrm{~Hz}, 1 \mathrm{H}), 6.72(\mathrm{~d}, J=8.0 \mathrm{~Hz}, 2 \mathrm{H}), 7.26-7.44(\mathrm{~m}, 5 \mathrm{H})$, 7.47-7.54 (m, 1H); ${ }^{13} \mathbf{C}$ NMR (125.7 $\left.\mathrm{MHz} \mathrm{CDCl}_{3}\right) \delta$ 28.2, 39.9, 55.3, 62.6, 77.0, 109.5, 110.7, 113.5, 128.7, 128.9, 129.0, 130.4, 136.9, 142.4, 150.8, 154.7, 158.4. HRMS Ca1cd for $\mathrm{C}_{25} \mathrm{H}_{28} \mathrm{~N}_{2} \mathrm{O}_{6}$ : 452.1947; Found: 452.1948. The enantiomeric ratio of the minor diastereomer was determined by CSP-HPLC on a Whelk-O column with $2 \%$ IPA/hexanes mobile phase and a flow rate of $0.4 \mathrm{~mL} / \mathrm{min}: \mathrm{rt}=42.3 \mathrm{~min}(4 \%), 45.1 \mathrm{~min}(96 \%)$.

(4-Methoxy-phenyl)-(3-nitro-l-phenyl-2-thiophen-2-yl-propyl)-carbamic Acid tertButyl Ester (44). The general lithiation procedure was followed using 28 (0.500 g, $1.60 \mathrm{mmol})$ and 33 (0.370 g, $2.40 \mathrm{mmol})$. Purification by column chromatography (85:15 hexanes/EtOAc) gave $44(0.664 \mathrm{~g}, 89 \%, 89: 11 \mathrm{dr})$. The diastereomers were separated by preparative HPLC (9:1 
hexanes/EtOAc). The major diastereomer was less retained. Major diastereomer: ${ }^{1} \mathbf{H}$ NMR $\left(500 \mathrm{MHz}, \mathrm{CDCl}_{3}\right) \delta 1.36(\mathrm{~s}, 9 \mathrm{H}), 3.80(\mathrm{~s}, 3 \mathrm{H}), 4.63(\mathrm{~m}, 1 \mathrm{H}), 4.94(\mathrm{t}, J=11.5 \mathrm{~Hz}, 1 \mathrm{H}), 5.18(\mathrm{~d}$, $J=10 \mathrm{~Hz}, 1 \mathrm{H}), 5.88(\mathrm{~s}, 1 \mathrm{H}), 6.60(\mathrm{~s}, 2 \mathrm{H}), 6.63-6.80(\mathrm{~m}, 4 \mathrm{H}), 6.70-7.20(\mathrm{~m}, 6 \mathrm{H}) ;{ }^{13} \mathbf{C} \mathbf{~ N M R}$ $\left(125.7 \mathrm{MHz}, \mathrm{CDCl}_{3}\right) \delta 28.2,42.3,55.3,62.8,80.7,113.7,125.1,126.6,126.9,127.8,129.4$, 130.7, 136.2, 139.7, 158.6. HRMS Calcd for $\mathrm{C}_{25} \mathrm{H}_{28} \mathrm{~N}_{2} \mathrm{O}_{5} \mathrm{~S}$ : 468.1719; Found: 468.1719. The enantiomeric ratio of the major diastereomer was determined by CSP-HPLC on a Chiral Pak AD column with $1 \% \mathrm{IPA} / \mathrm{hexanes}$ mobile phase and a flow rate of $0.5 \mathrm{~mL} / \mathrm{min}: \mathrm{rt}=41.0 \mathrm{~min}(3 \%)$, $52.8 \mathrm{~min}(97 \%)$. Minor diastereomer: ${ }^{1} \mathbf{H}$ NMR $\left(500 \mathrm{MHz}, \mathrm{CDCl}_{3}\right) \delta 1.28(\mathrm{~s}, 9 \mathrm{H}), 3.79$ (s, $3 \mathrm{H}), 4.36(\mathrm{~d}, J=7.0 \mathrm{~Hz}, 2 \mathrm{H}), 4.56(\mathrm{~s}, 1 \mathrm{H}), 5.52(\mathrm{~s}, 1 \mathrm{H}), 6.31(\mathrm{~s}, 2 \mathrm{H}), 6.68(\mathrm{~d}, J=6.5 \mathrm{~Hz}, 2 \mathrm{H})$, $7.06(\mathrm{dd}, J=3.5,5.0 \mathrm{~Hz}, 1 \mathrm{H}), 7.17(\mathrm{~s}, 1 \mathrm{H}), 7.30-7.44(\mathrm{~m}, 6 \mathrm{H}),{ }^{13} \mathbf{C} \mathbf{~ N M R}\left(125.7 \mathrm{MHz}, \mathrm{CDCl}_{3}\right) \delta$ 28.2, 41.1, 55.3, 63.8, 80.2, 113.4, 125.2, 127.0, 127.2, 128.8, 128.9, 129.0, 137.4, 140.1, 158.5 . HRMS Ca1cd for $\mathrm{C}_{25} \mathrm{H}_{28} \mathrm{~N}_{2} \mathrm{O}_{5} \mathrm{~S}: 468.1719$; Found: 468.1718 .

\section{Furan-2-yl-3-nitro-2-phenyl-propyl)-(4-methoxy-phenyl)-carbamic Acid tert-Butyl} Ester (45). The general lithiation procedure was followed using 29 (0.303 g, $1.0 \mathrm{mmol})$ and $\mathbf{1 0}$ (0.220 g, $1.50 \mathrm{mmol})$. Purification by column chromatography (85:15 hexanes/EtOAc) gave $\mathbf{4 5}$ $(0.440$ g, $98 \%,>99: 1 \mathrm{dr})$. Major diastereomer: ${ }^{1} \mathbf{H}$ NMR $\left(500 \mathrm{MHz}, \mathrm{CDCl}_{3}\right) \delta 1.48(\mathrm{~s}, 3 \mathrm{H})$, 3.64-3.74 (m, 1H), $3.81(\mathrm{~s}, 3 \mathrm{H}), 3.89-3.96(\mathrm{~m}, 1 \mathrm{H}), 4.70-4.78(\mathrm{~m}, 2 \mathrm{H}), 5.07(\mathrm{t}, J=2.5 \mathrm{~Hz}, 1 \mathrm{H})$, $5.97(\mathrm{~s}, 1 \mathrm{H}), 6.34(\mathrm{~s}, 1 \mathrm{H}), 6.84-6.88(\mathrm{~m}, 2 \mathrm{H}), 7.15-7.40(\mathrm{~m}, 9 \mathrm{H}) ;{ }^{13} \mathbf{C} \mathbf{~ N M R}\left(125.7 \mathrm{MHz}, \mathrm{CDCl}_{3}\right)$ $\delta$ 28.1, 48.5, 49.5, 55.3 75.7, 81.1, 102.6, 106.5, 113.5, 127.5, 127.6, 128.0, 128.9, 134.6, 136.9, 145.6, 153.4, 157.4. HRMS Calcd for $\mathrm{C}_{25} \mathrm{H}_{28} \mathrm{~N}_{2} \mathrm{O}_{6}$ : 451.1869; Found: 451.1868. The enantiomeric ratio of the major diastereomer was determined by CSP-HPLC on a Whelk-O column with $6 \%$ IPA/hexanes mobile phase and a flow rate of $1.5 \mathrm{~mL} / \mathrm{min}: \mathrm{rt}=35.9 \mathrm{~min}(>99$ $\%), 38.3 \min (<1 \%)$.

(4-Methoxy-phenyl)-[3-(2-nitro-cyclohexyl)-3-phenyl-propenyl]-carbamic Acid tertButyl Ester (48). The general lithiation procedure was followed using $2(0.200 \mathrm{~g}, 0.588 \mathrm{mmol})$ and $46(0.093 \mathrm{~mL}, 0.823 \mathrm{mmol})$ except for the reaction was quenched at $-78{ }^{\circ} \mathrm{C}$ with saturated $\mathrm{NH}_{4} \mathrm{Cl}$ and the workup involved washing with saturated $\mathrm{NH}_{4} \mathrm{Cl}$ rather than brine/ $\mathrm{H}_{2} \mathrm{O}$. Purification by column chromatography (5:1 pet ether/EtOAc) gave $\mathbf{4 8}(0.179 \mathrm{~g}, 65 \%, 84: 16 \mathrm{dr})$. 
Major diastereomer: ${ }^{1} \mathbf{H}$ NMR (acetone- $\left.d_{6}, 400 \mathrm{MHz}\right) \delta 0.89\left(\mathrm{~m}, 2 \mathrm{H}\right.$, ring $\left.\mathrm{C}_{2}\right), 1.10(\mathrm{~m}, 1 \mathrm{H}$, ring $\mathrm{C} \underline{\mathrm{H}}), 1.26-1.62\left(\mathrm{~m}, 3 \mathrm{H}\right.$, ring $\left.\mathrm{C}_{2}, \mathrm{C} \underline{\mathrm{H}}\right), 1.44$ (s, $\left.9 \mathrm{H}, \mathrm{Ot}-\underline{\mathrm{Bu}}\right), 1.89$ (m, $2 \mathrm{H}$, ring $\underline{\mathrm{C}}_{2}$ ), 2.29 (br d, $J=14.6 \mathrm{~Hz}, 1 \mathrm{H}$, ring-C $\underline{H C H P h}$ ), 3.24 (br m, 1H, C파), 3.84 (s, 3H, OMe), 5.07 (br m, 1H, $\left.\mathrm{O}_{2} \mathrm{NC} \underline{H}\right), 5.15(\mathrm{t}, J=9.8 \mathrm{~Hz}, 1 \mathrm{H}, \mathrm{C} \underline{H}=\mathrm{CHN}), 6.51(\mathrm{~m}, 1 \mathrm{H}, \mathrm{Ph} \underline{\mathrm{H}}), 6.65(\mathrm{~d}, J=9.8 \mathrm{~Hz}, 1 \mathrm{H}$, $\mathrm{CH}=\mathrm{C} \underline{H N}), 6.82(\mathrm{~m}, 2 \mathrm{H}, \mathrm{Ph} \underline{\mathrm{H}}), 6.99(\mathrm{~m}, 1 \mathrm{H}, \mathrm{Ph} \underline{\mathrm{H}}), 7.10(\mathrm{~m}, 3 \mathrm{H}, \mathrm{Ph} \underline{\mathrm{H}}) ;{ }^{13} \mathbf{C}$ NMR (acetone- $d_{6}$, $125.6 \mathrm{MHz}) \delta 20.5\left(\mathrm{CH}_{2}\right), 24.2\left(\mathrm{CH}_{2}\right), 24.9\left(\mathrm{CH}_{2}\right), 27.6(\mathrm{CH}), 31.2\left(\mathrm{CH}_{2}\right), 44.4(\mathrm{CH}), 45.5(\mathrm{CH})$, $55.2\left(\mathrm{CH}_{3}\right), 80.5(\mathrm{C}), 83.6(\mathrm{CH}), 114.0(\mathrm{CH}), 126.3(\mathrm{CH}), 127.9(\mathrm{CH}), 128.0(\mathrm{CH}), 128.5(\mathrm{CH})$, $129.6(\mathrm{CH}), 135.7(\mathrm{C}), 141.8(\mathrm{C}), 153.2(\mathrm{CH}), 158.0(\mathrm{C})$. Anal. Calcd for $\mathrm{C}_{27} \mathrm{H}_{34} \mathrm{~N}_{2} \mathrm{O}_{5}$ : C, 69.50; H, 7.35; N, 6.00. Found C, 69.50; H, 7.39; N, 5.99. $[\alpha]_{\mathrm{D}}^{20}:+193.7^{\circ}(c=1.60$, EtOAc $)$. The enantiomeric ratio of the major diastereomer was determined by CSP-HPLC on a Whelk-O column with $10 \% \mathrm{IPA} / \mathrm{hexanes}$ mobile phase and a flow rate of $1.0 \mathrm{~mL} / \mathrm{min}: \mathrm{rt}=9.10 \mathrm{~min}(>99$ $\%), 11.5 \mathrm{~min}(<1 \%)$. Minor diastereomer: ${ }^{1} \mathbf{H}$ NMR (acetone- $\left.d_{6}, 500 \mathrm{MHz}\right) \delta 0.71(\mathrm{~m}, 1 \mathrm{H}$, ring $\mathrm{C} \underline{\mathrm{H}}), 1.22(\mathrm{~m}, 2 \mathrm{H}$, ring $\mathrm{C} \underline{\mathrm{H}}), 1.39(\mathrm{~m}, 1 \mathrm{H}$, ring $\mathrm{C} \underline{\mathrm{H}}), 1.39(\mathrm{~s}, 9 \mathrm{H}, \mathrm{Ot}-\underline{\mathrm{Bu}}), 1.51(\mathrm{~m}, 1 \mathrm{H}$, ring $\mathrm{C} \underline{\mathrm{H}})$, $1.66(\mathrm{~m}, 2 \mathrm{H}$, ring $\mathrm{CH}), 1.86(\mathrm{~m}, 1 \mathrm{H}$, ring $\mathrm{CH}), 2.28(\mathrm{~m}, 1 \mathrm{H}$, ring-CHCHPh), $3.12(\mathrm{dd}, J=9.2$, $6.7 \mathrm{~Hz}, 1 \mathrm{H}, \mathrm{CHPh}$ ), 3.81 (s, 3H, OMe), 4.21 (td, $\left.J=9.6,4.3 \mathrm{~Hz}, 1 \mathrm{H}, \mathrm{O}_{2} \mathrm{NC} \underline{\mathrm{H}}\right), 5.26$ (t, $J=9.9$

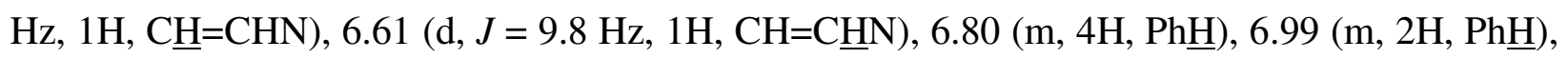
$7.17(\mathrm{~m}, 3 \mathrm{H}, \mathrm{Ph} \underline{\mathrm{H}}) ;{ }^{13} \mathrm{C}$ NMR (acetone- $\left.d_{6}, 125.6 \mathrm{MHz}\right) \delta 23.5\left(\mathrm{CH}_{2}\right), 25.5\left(\mathrm{CH}_{2}\right), 27.6(\mathrm{CH})$, $31.45\left(\mathrm{CH}_{2}\right), 43.0(\mathrm{CH}), 44.7(\mathrm{CH}), 55.1\left(\mathrm{CH}_{3}\right), 80.6(\mathrm{C}), 87.7(\mathrm{CH}), 114.1(\mathrm{CH}), 126.5(\mathrm{CH})$, $128.0(\mathrm{CH}), 128.4(\mathrm{CH}), 128.7(\mathrm{CH}), 134.9(\mathrm{C}), 140.3(\mathrm{C}), 153.2(\mathrm{CH}), 160.9(\mathrm{C})$.

\section{(4-Methoxy-phenyl)-(5-nitro-3,4-diphenyl-hex-1-enyl)-carbamic Acid tert-Butyl}

Ester (49). The general lithiation procedure was followed using $2(0.150 \mathrm{~g}, 0.441 \mathrm{mmol})$ and 47 (0.094 g, $0.573 \mathrm{mmol}$ ) except for the reaction was quenched at $-78{ }^{\circ} \mathrm{C}$ with saturated $\mathrm{NH}_{4} \mathrm{Cl}$ and the workup involved washing with saturated $\mathrm{NH}_{4} \mathrm{Cl}$ rather than brine $/ \mathrm{H}_{2} \mathrm{O}$. Purification by column chromatography (4:1 pet ether/EtOAc) gave 49 (0.179 g, $65 \%, 55: 45$ dr). Major diastereomer: $\mathrm{mp} 183-184{ }^{\circ} \mathrm{C} ;{ }^{1} \mathbf{H}$ NMR (acetone- $\left.d_{6}, 500 \mathrm{MHz}\right) \delta 1.43(\mathrm{~s}, 9 \mathrm{H}, \mathrm{Ot}$ - $\underline{\mathrm{Bu}}$ ), $1.54(\mathrm{~d}, J$ $\left.=6.6 \mathrm{~Hz}, 3 \mathrm{H}, \mathrm{CHCH}_{3}\right), 3.38$ (dd, $\left.J=9.0,5.8 \mathrm{~Hz}, 1 \mathrm{H}, \mathrm{CHPh}\right), 3.85$ (s, 3H, OMe), $4.0(\mathrm{br} \mathrm{m}, 1 \mathrm{H}$, $\mathrm{C} \underline{H P h}$ ), 5.06 (quint, $\left.J=6.6 \mathrm{~Hz}, 1 \mathrm{H}, \mathrm{O}_{2} \mathrm{NC} \underline{\mathrm{H}}\right), 5.25(\mathrm{t}, J=9.3 \mathrm{~Hz}, 1 \mathrm{H}, \mathrm{C} \underline{\mathrm{H}}=\mathrm{CHN}), 6.47(\mathrm{~m}, 2 \mathrm{H}$, $\mathrm{Ph} \underline{\mathrm{H}}), 6.73(\mathrm{~d}, J=8.9 \mathrm{~Hz}, 1 \mathrm{H}, \mathrm{CH}=\mathrm{C} \underline{\mathrm{HN}}), 6.83(\mathrm{~m}, 2 \mathrm{H}, \mathrm{Ph} \underline{\mathrm{H}}), 6.94(\mathrm{~m}, 5 \mathrm{H}, \mathrm{Ph} \underline{\mathrm{H}}), 7.05$ (m, 3H, $\mathrm{Ph} \underline{\mathrm{H}}), 7.24(\mathrm{~m}, 2 \mathrm{H}, \mathrm{Ph} \underline{\mathrm{H}}) ;{ }^{13} \mathrm{C}$ NMR (acetone- $\left.d_{6}, 125.6 \mathrm{MHz}\right) \delta 18.1\left(\mathrm{CH}_{3}\right), 27.7\left(\mathrm{CH}_{3}\right), 43.6$ 
$(\mathrm{CH}), 55.2(\mathrm{CH}), 55.4\left(\mathrm{CH}_{3}\right), 80.6(\mathrm{C}), 84.6(\mathrm{CH}), 114.2(\mathrm{CH}), 126.2(\mathrm{CH}), 127.3(\mathrm{CH}), 127.8$ (CH), $127.8(\mathrm{CH}), 128.4(\mathrm{CH}), 129.7(\mathrm{CH}), 135.9(\mathrm{C}), 136.7(\mathrm{C}), 140.8(\mathrm{C}), 153.4(\mathrm{CH}), 158.2$ (C). Anal. Calcd for $\mathrm{C}_{30} \mathrm{H}_{34} \mathrm{~N}_{2} \mathrm{O}_{5}$ : C, 71.69; H, 6.82; N, 5.57. Found C, 71.42; H, 6.83; N, 5.33. The enantiomeric ratio of the major diastereomer was determined by CSP-HPLC on a Whelk-O column with $10 \% \mathrm{IPA} / \mathrm{hexanes}$ mobile phase and a flow rate of $1.0 \mathrm{~mL} / \mathrm{min}: \mathrm{rt}=11.7 \mathrm{~min}(>99$ $\%), 17.5 \min (<1 \%)$. Minor diastereomer: ${ }^{1} \mathbf{H}$ NMR (acetone- $\left.d_{6}, 500 \mathrm{MHz}\right) \delta 1.31(\mathrm{~d}, J=6.6$ $\left.\mathrm{Hz}, 3 \mathrm{H}, \mathrm{CHCH}_{3}\right), 1.44$ (s, 9H, Ot-Bu), 3.66 (t, $J=10.5 \mathrm{~Hz}, 1 \mathrm{H}, \mathrm{C} \underline{\mathrm{HPh}}$ ), 3.79 (dd, $J=10.3,5.8$ $\mathrm{Hz} 1 \mathrm{H}, \mathrm{C} \underline{\mathrm{HPh}}$ ), 3.86 (s, 3H, OMe), 5.06 (quint, $J=6.6 \mathrm{~Hz}, 1 \mathrm{H}, \mathrm{O}_{2} \mathrm{NC} \underline{\mathrm{H}}$ ), 5.31 (t, $J=9.3 \mathrm{~Hz}$, 1H, $\mathrm{C} \underline{H}=\mathrm{CHN}), 6.57(\mathrm{~m}, 2 \mathrm{H}, \mathrm{Ph} \underline{\mathrm{H}}), 6.72(\mathrm{~d}, J=9.7 \mathrm{~Hz}, 1 \mathrm{H}, \mathrm{CH}=\mathrm{C} \underline{H N}), 6.77$ (m, 2H, Ph్) , $6.95(\mathrm{~m}, 5 \mathrm{H}, \mathrm{Ph} \underline{\mathrm{H}}), 7.06(\mathrm{~m}, 3 \mathrm{H}, \mathrm{Ph} \underline{\mathrm{H}}), 7.22(\mathrm{~m}, 2 \mathrm{H}, \mathrm{Ph} \underline{\mathrm{H}}) ;{ }^{13} \mathbf{C}$ NMR (acetone- $d_{6}, 125.6 \mathrm{MHz}$ ) $\delta 14.7\left(\mathrm{CH}_{3}\right), 27.6\left(\mathrm{CH}_{3}\right), 44.0(\mathrm{CH}), 54.7(\mathrm{CH}), 55.3\left(\mathrm{CH}_{3}\right), 80.9(\mathrm{C}), 84.9(\mathrm{CH}), 114.4(\mathrm{CH})$, $126.2(\mathrm{CH}), 127.3(\mathrm{CH}), 127.8(\mathrm{CH}), 128.0(\mathrm{CH}), 128.3(\mathrm{CH}), 129.81(\mathrm{CH}), 135.6(\mathrm{C}), 136.0$ (C), 140.8 (C), $153.4(\mathrm{CH}), 158.4$ (C). Anal. Calcd for $\mathrm{C}_{30} \mathrm{H}_{34} \mathrm{~N}_{2} \mathrm{O}_{5}$ : C, 71.69; H, 6.82; N, 5.57. Found C, 71.36; H, 6.87; N, 5.52 .

\section{(4-Methoxy-phenyl)-[(2-nitro-cyclohexyl)-phenyl-methyl]-carbamic Acid tert-Butyl}

Ester (50) The general 1ithiation procedure was followed using $28(0.310 \mathrm{~g}, 1.00 \mathrm{mmol})$ and $\mathbf{4 6}$ (0.190 g, $1.50 \mathrm{mmol})$. Purification by column chromatography (95:5 hexanes/EtOAc) gave $\mathbf{5 0}$ $(0.440 \mathrm{~g}, 77 \%, 68: 32 \mathrm{dr})$. The diastereomers were separated by flash column chromatography. The major diastereomer was less retained. Major diastereomer: ${ }^{1} \mathbf{H}$ NMR $\left(400 \mathrm{MHz}, \mathrm{CDCl}_{3}\right) \delta$ 1.34, (s, 9H), 1.15-1.58 (m, 4H), 2.32-2.52 (m, 2H), 3.12- 3.22 (m, 1H), 3.79 (s, 3H), 4.95 (d, $J$ $=3.2 \mathrm{~Hz}, 1 \mathrm{H}), 5.77(\mathrm{~d}, J=12.0 \mathrm{~Hz}, 1 \mathrm{H}), 6.58(\mathrm{~d}, J=7.6 \mathrm{~Hz}, 2 \mathrm{H}), 6.71(\mathrm{~d}, J=8.8 \mathrm{~Hz}, 2 \mathrm{H}), 6.93$ $(\mathrm{d}, J=6.8 \mathrm{~Hz}, 2 \mathrm{H}), 7.16-7.27(\mathrm{~m}, 3 \mathrm{H}) ;{ }^{13} \mathbf{C} \mathbf{~ N M R}\left(100 \mathrm{MHz}, \mathrm{CDCl}_{3}\right) \delta 20.0,21.2,23.6,25.3$, 25.9, 28.2, 38.1, 55.2, 60.9, 80.5, 84.2, 113.4, 127.5, 127.9, 128.1, 129.4, 131.0, 131.1, 136.8, 156.0, 158.4. HRMS Calcd for $\mathrm{C}_{25} \mathrm{H}_{32} \mathrm{~N}_{2} \mathrm{O}_{5}$ : 440.2311; Found: 440.2311. The enantiomeric ratio of the major diastereomer was determined by CSP-HPLC on a Whelk-O column with $2.5 \%$ IPA/hexanes mobile phase and a flow rate of $0.5 \mathrm{~mL} / \mathrm{min}: \mathrm{rt}=18.9 \mathrm{~min}(2 \%), 20.5 \mathrm{~min}(98 \%)$.

Minor diastereomer: ${ }^{1} \mathbf{H}$ NMR $\left(400 \mathrm{MHz}, \mathrm{CDCl}_{3}\right) \delta$ 1.05-2.05 (m, 16H), $4.03(\mathrm{~d}, J=14.0 \mathrm{~Hz}$, 1H), $3.12(\mathrm{~s}, 1 \mathrm{H}), 3.77$ (s, 3H), $4.43(\mathrm{~s}, 1 \mathrm{H}), 5.29(\mathrm{~s}, 1 \mathrm{H}), 6.54(\mathrm{~d}, J=8.4 \mathrm{~Hz}, 2 \mathrm{H}), 6.72$ (d, $J=$ 8.0 Hz, 2H), 7.24-7.40 (m, 5H); ${ }^{13} \mathbf{C}$ NMR $\left(100 \mathrm{MHz}, \mathrm{CDCl}_{3}\right) \delta$ 20.3, 24.5, 25.0, 28.2, 31.6, 41.6, 55.3, 68.4, 80.1, 83.2, 113.6, 127.8, 128.1, 128.3, 129.2, 140.1, 155.6, 131.1, 136.8, 156.0, 
158.4. HRMS Calcd for $\mathrm{C}_{25} \mathrm{H}_{32} \mathrm{~N}_{2} \mathrm{O}_{5}$ : 440.2311; Found: 440.2312. The enantiomeric ratio of the minor diastereomer was determined by CSP-HPLC on a Whelk-O column with $2.5 \%$ IPA/hexanes mobile phase and a flow rate of $0.5 \mathrm{~mL} / \mathrm{min}: \mathrm{rt}=17.2 \mathrm{~min}(95 \%), 57.1 \mathrm{~min}(5 \%)$.

(4-Methoxy-phenyl)-(3-nitro-l,2-diphenyl-butyl)-carbamic Acid tert-Butyl Ester (51). The general lithiation procedure was followed using $28(0.500 \mathrm{~g}, 1.60 \mathrm{mmol})$ and $47(0.390 \mathrm{~g}$, $2.40 \mathrm{mmol})$. Purification by column chromatography (9:1 hexanes/EtOAc) gave the $\mathbf{5 1}(0.550 \mathrm{~g}$, $72 \%, 60: 40 \mathrm{dr}$ ). The diastereomers were separated by preparative HPLC (93:7 hexanes/EtOAc).

The major diastereomer was less retained. Major diastereomer: ${ }^{1} \mathbf{H}$ NMR $\left(500 \mathrm{MHz}, \mathrm{CDCl}_{3}\right) \delta$ $1.36(\mathrm{~s}, 9 \mathrm{H}), 1.61$ (d, $J=6.5 \mathrm{~Hz}, 3 \mathrm{H}), 3.80(\mathrm{~s}, 3 \mathrm{H}), 4.35(\mathrm{~d}, J=11.0 \mathrm{~Hz}, 1 \mathrm{H}), 5.38(\mathrm{~s}, 1 \mathrm{H}), 6.31$ $(\mathrm{d}, J=11.0 \mathrm{~Hz}, 1 \mathrm{H}), 6.52-7.04(\mathrm{~m}, 14 \mathrm{H}) ;{ }^{13} \mathbf{C} \mathbf{N M R}\left(125.7 \mathrm{MHz}, \mathrm{CDCl}_{3}\right) \delta$ 13.0, 28.1, 50.0, 55.2, 59.8, 80.6, 84.0, 113.4, 127.4, 127.9, 128.1, 129.6, 129.6, 130.4, 131.1, 134.1, 137.1, 156.2, 158.5. Anal. Calcd for $\mathrm{C}_{28} \mathrm{H}_{32} \mathrm{~N}_{2} \mathrm{O}_{5}: \mathrm{C}, 70.57 ; \mathrm{H}, 6.77 ; \mathrm{N}, 5.88$. Found: C, 70.57; H, 6.77; N, 5.88. The enantiomeric ratio of the major diastereomer was determined by CSP-HPLC on a Whelk-O column with $2 \% \mathrm{IPA} /$ hexanes mobile phase and a flow rate of $0.4 \mathrm{~mL} / \mathrm{min}$ : $\mathrm{rt}=24.5$ $\min (12 \%), 27.3 \min (88 \%)$. Minor diastereomer: ${ }^{1} \mathbf{H} \mathbf{N M R}\left(500 \mathrm{MHz}, \mathrm{CDCl}_{3}\right) \delta 1.38(\mathrm{~s}, 9 \mathrm{H})$, $1.63(\mathrm{~d}, J=7.0 \mathrm{~Hz}, 3 \mathrm{H}), 3.81(\mathrm{~s}, 3 \mathrm{H}), 4.20-4.80(\mathrm{~m}, 1 \mathrm{H}), 5.50(\mathrm{~s}, 2 \mathrm{H}), 6.75-7.45(\mathrm{~m}, 14 \mathrm{H})$. Anal. Ca1cd for $\mathrm{C}_{28} \mathrm{H}_{32} \mathrm{~N}_{2} \mathrm{O}_{5}:$ C, 70.57; H, 6.77; N, 5.88. Found: C, 69.13; H, 5.99; N, 5.93.

\section{Conversion of 23 to 58 .}

Part 1: 5-Nitro-3-phenyl-pentanoic Acid Methyl Ester. The general procedure for conversion to 5-nitro-pentanoic acid methyl esters was followed using 23 (2.95 g, $7.16 \mathrm{mmol})$ except esterification was achieved by stirring the crude acid in methanol $(100 \mathrm{~mL})$ with $\mathrm{HCl}$ (17.8 $\mathrm{mL}$ of a $2 \mathrm{~N}$ solution in $\mathrm{Et}_{2} \mathrm{O}, 35.6 \mathrm{mmol}$ ). Concentration and purification by column chromatography (4:1 pet ether/EtOAc) gave the title compound $(1.40 \mathrm{~g}, 82 \%)$ as an oil. Major diastereomer: ${ }^{1} \mathbf{H} \mathbf{N M R}\left(\mathrm{CDCl}_{3}, 500 \mathrm{MHz}\right) \delta 2.23\left(\mathrm{~m}, 1 \mathrm{H}, \mathrm{CH}_{2} \mathrm{CH} 2 \mathrm{CHPh}\right), 2.50(\mathrm{dq}, J=13.9$, $\left.4.3 \mathrm{~Hz}, 1 \mathrm{H}, \mathrm{CH}_{2} \mathrm{C} \underline{\mathrm{H}} 2 \mathrm{CHPh}\right), 2.68$ (d, $\left.J=7.3 \mathrm{~Hz}, 1 \mathrm{H}, \underline{\mathrm{C}}_{2} \mathrm{CO}_{2} \mathrm{Me}\right), 3.19(\mathrm{~m}, 1 \mathrm{H}, \mathrm{C} \underline{\mathrm{HPh}}), 3.61(\mathrm{~s}$, $\left.3 \mathrm{H}, \mathrm{OC}_{\underline{3}}\right), 4.19\left(\mathrm{~m}, 2 \mathrm{H}, \underline{\mathrm{C}}_{2} \mathrm{NO}_{2}\right), 7.18$ (m, 2H, $\left.\mathrm{Ph} \underline{\mathrm{H}}\right), 7.26$ (m, 1H, PhH $), 7.33$ (m, 2H, PhH); ${ }^{13} \mathbf{C}$ NMR $\left(\mathrm{CDCl}_{3}, 125.6 \mathrm{MHz}\right) \delta 33.0(\mathrm{CH}), 39.1(\mathrm{CH}), 41.1\left(\mathrm{CH}_{2}\right), 51.7\left(\mathrm{CH}_{3}\right), 73.4\left(\mathrm{CH}_{2}\right)$, $127.3(\mathrm{CH}), 127.5(\mathrm{CH}), 129.0(\mathrm{CH}), 141.0(\mathrm{C}), 171.8(\mathrm{C})$. HRMS-FAB $(\mathrm{M}+1)$ Calcd for $\mathrm{C}_{12} \mathrm{H}_{16} \mathrm{NO}_{4}$ : 238.1079; Found: 238.1078. 
Part 2: 4-Phenylpiperidin-2-one (58). The general hydrogenation-cyclization procedure was followed using 5-nitro-3-phenyl-pentanoic acid methyl ester (1.35 g, $5.69 \mathrm{mmol})$. Filtration of the catalyst followed by concentration gave $58(0.960 \mathrm{~g}, 96 \%)$ as a white solid: $\mathrm{mp}$ 154-155 ${ }^{\circ} \mathrm{C} .{ }^{1} \mathbf{H}$ NMR $\left(\mathrm{CDCl}_{3}, 500 \mathrm{MHz}\right) \delta 1.92\left(\mathrm{~m}, 1 \mathrm{H}, \mathrm{C}_{2}{ }_{2} \mathrm{CHPh}\right), 2.08\left(\mathrm{~m}, 1 \mathrm{H}, \mathrm{CH}_{2} \mathrm{CHPh}\right)$, $2.48\left(\mathrm{dd}, J=17.6,11.2 \mathrm{~Hz}, 1 \mathrm{H}, \mathrm{CHCH}_{2} \mathrm{CO}\right), 2.68\left(\mathrm{ddd}, J=17.8,5.3,1.7 \mathrm{~Hz}, 1 \mathrm{H}, \mathrm{CHC}_{2} \mathrm{CO}\right.$ ), $3.10(\mathrm{tdd}, J=11.2,5.2,3.2 \mathrm{~Hz}, 1 \mathrm{H}, \mathrm{CHPh}), 3.39\left(\mathrm{dd}, J=7.7,3.4 \mathrm{~Hz}, 2 \mathrm{H}, \mathrm{CHC}_{2} \underline{\mathrm{N}}^{\mathrm{N}}\right), 7.10$ (br s,

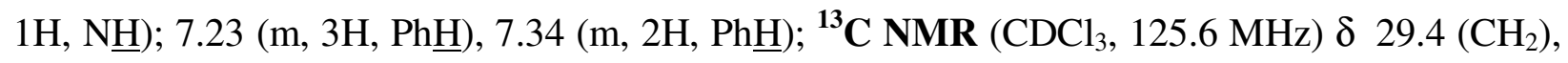
$38.3(\mathrm{CH}), 41.2\left(\mathrm{CH}_{2}\right), 126.5(\mathrm{CH}), 126.8(\mathrm{CH}), 128.7(\mathrm{CH}), 143.5(\mathrm{C}), 172.4(\mathrm{C})$. HRMS-FAB $(\mathrm{M}+1)$ Calcd for $\mathrm{C}_{11} \mathrm{H}_{14} \mathrm{NO}$ : 176.1075; Found: 176.1075.

\section{Conversion of 17 to 62 .}

Part 2: 3,4-Diphenylpyrrolidin-2-one (62). The general hydrogenation-cyclization procedure was followed using 4-nitro-2,3-diphenyl-butyric acid methyl ester (0.095 g, 0.317 mmol). Purification by column chromatography gave $62(0.063 \mathrm{~g}, 85 \%)$ as an oil. The ${ }^{1} \mathrm{H}$ NMR spectrum was consistent with reported literature values. ${ }^{4}[\alpha]_{\mathrm{D}}^{20}:-243.8^{\circ}\left(c=0.64, \mathrm{CHCl}_{3}\right)$

\section{Conversion of 25 to 63 .}

Part 1: 2-Methyl-4-nitro-3-phenyl-butyric Acid Methyl Ester. The general procedure for conversion to 4-nitro-butyric acid methyl esters was followed using 25 (3.00 g, $7.03 \mathrm{mmol}$ ). Purification by column chromatography (5:1 pet ether/EtOAc) gave the title compound (1.19 g, $71 \%,>99: 1 \mathrm{dr})$ a white solid: $\mathrm{mp} 53-55^{\circ} \mathrm{C} .{ }^{1} \mathbf{H} \mathbf{~ N M R}\left(\mathrm{CDCl}_{3}, 500 \mathrm{MHz}\right) \delta 1.03(\mathrm{~d}, J=7.1 \mathrm{~Hz}$, $\left.3 \mathrm{H}, \mathrm{CHCH}_{3}\right), 2.78\left(\mathrm{dq}, J=9.8,7.1 \mathrm{~Hz}, 1 \mathrm{H}, \mathrm{CHCH}_{3}\right), 3.71(\mathrm{~m}, 1 \mathrm{H}, \mathrm{C} \underline{\mathrm{HPh}}), 3.72\left(\mathrm{~s}, 3 \mathrm{H}, \mathrm{OC}_{\underline{3}}\right)$, $4.70\left(\mathrm{~d}, J=7.3 \mathrm{~Hz}, 2 \mathrm{H}, \mathrm{NO}_{2} \mathrm{CH}_{2}\right), 7.17(\mathrm{~m}, 4 \mathrm{H}, \mathrm{Ph} \underline{\mathrm{H}}), 7.31(\mathrm{~m}, 6 \mathrm{H}, \mathrm{Ph} \underline{\mathrm{H}}) ;{ }^{13} \mathrm{C} \mathrm{NMR}\left(\mathrm{CDCl}_{3}\right.$, $125.6 \mathrm{MHz}) \delta 15.9\left(\mathrm{CH}_{3}\right), 42.6(\mathrm{CH}), 46.7(\mathrm{CH}), 52.1\left(\mathrm{CH}_{3}\right), 78.5\left(\mathrm{CH}_{2}\right), 127.9(\mathrm{CH}), 128.0$ $(\mathrm{CH}), 129.0(\mathrm{CH}), 137.0(\mathrm{C}), 175.0(\mathrm{C})$. Anal. Calcd for $\mathrm{C}_{12} \mathrm{H}_{15} \mathrm{NO}_{4}$ : C, 60.75; H, 6.37; N, 5.90. Found C, 60.81; H, 6.27; N, 5.99. $[\alpha]_{\mathrm{D}}^{20}:+28.5^{\circ}\left(c=1.0, \mathrm{CHCl}_{3}\right)$.

Part 2: 3-Methyl-4-phenyl-pyrrolidin-2-one (63). The general hydrogenationcyclization procedure was followed using 2-methyl-4-nitro-3-phenyl-butyric acid methyl ester (0.095 g, $0.317 \mathrm{mmol})$. Recrystallization from hexanes/EtOAc gave $62(0.499 \mathrm{~g}, 68 \%)$ as white 
crystals: mp 100-101 ${ }^{\circ} \mathrm{C}$. lit. ${ }^{5} \mathrm{mp}$ 100-101 ${ }^{\circ} \mathrm{C}$. The ${ }^{1} \mathrm{H}$ NMR and ${ }^{13} \mathrm{C}$ NMR spectra were consistent with reported literature values. ${ }^{5}[\alpha]_{\mathrm{D}}^{20}:-68.3^{\circ}\left(c=1.0, \mathrm{CHCl}_{3}\right)$. lit. $^{5}$ (enantiomer) $[\alpha]_{\mathrm{D}}^{20}:+73.5^{\circ}\left(c=0.95, \mathrm{CHCl}_{3}\right)$.

\section{Conversion of 26 to 64.}

Part 1: 2,5-Dimethyl-3-nitromethyl-hexanoic Acid Methyl Ester. The general procedure for conversion to 4-nitro-butyric acid methyl esters was followed using 26 (2.86 g, $7.03 \mathrm{mmol})$. Purification by column chromatography (9:1 pet ether/EtOAc) gave the title compound $(1.14 \mathrm{~g}, 75 \%, 84: 16 \mathrm{dr})$ as an oil. Major diastereomer: ${ }^{1} \mathbf{H} \mathbf{~ N M R}\left(\mathrm{CDCl}_{3}, 500 \mathrm{MHz}\right)$ $\delta 0.86\left(\mathrm{~d}, J=6.5 \mathrm{~Hz}, 3 \mathrm{H}, \mathrm{CH}_{2} \mathrm{CHMe}_{2}\right), 0.87\left(\mathrm{~d}, J=6.6 \mathrm{~Hz}, 3 \mathrm{H}, \mathrm{CH}_{2} \mathrm{CHMe}_{2}\right), 0.88(\mathrm{~m}, 1 \mathrm{H}$, $\mathrm{C}_{2} \mathrm{CHMe}_{2}$ ), 1.14 (m, 1H, $\underline{\mathrm{C}}_{2} \mathrm{CHMe}_{2}$ ), 1.16 (d, $J=7.0 \mathrm{~Hz}, 3 \mathrm{H}, \mathrm{CHMe}$ ), 1.59 (nonet, $J=6.6$ $\mathrm{Hz}, 1 \mathrm{H}, \mathrm{C}_{\mathrm{HMe}}$ ), 2.61 (m, 1H, $\left.\mathrm{NO}_{2} \mathrm{CH}_{2} \mathrm{C} \underline{\mathrm{H}}\right), 2.67$ (m, 1H, $\underline{\mathrm{HMe}}$ ), 3.66 (s, 3H, OMe), 4.35 (dd, $\left.J=12.4,6.4 \mathrm{~Hz}, 1 \mathrm{H}, \mathrm{NO}_{2} \mathrm{C}_{2}\right), 4.45\left(\mathrm{dd}, J=12.5,6.7 \mathrm{~Hz}, 1 \mathrm{H}, \mathrm{NO}_{2} \mathrm{C}_{2}\right),{ }^{13} \mathbf{C} \mathbf{N M R}\left(\mathrm{CDCl}_{3}\right.$, $125.6 \mathrm{MHz}) \delta 13.0\left(\mathrm{CH}_{3}\right), 22.1\left(\mathrm{CH}_{3}\right), 22.5\left(\mathrm{CH}_{3}\right), 25.1(\mathrm{CH}), 37.7(\mathrm{CH}), 38.3\left(\mathrm{CH}_{2}\right), 40.0$ $(\mathrm{CH}), 51.7\left(\mathrm{CH}_{3}\right), 77.2\left(\mathrm{CH}_{2}\right), 174.8(\mathrm{C})$. HRMS-FAB (M+1) Calcd for $\mathrm{C}_{10} \mathrm{H}_{20} \mathrm{NO}_{4}$ : 218.1392; Found: 218.1391.

Part 2: 4-iso-Butyl-3-methyl-pyrrolidin-2-one (64). The general hydrogenationcyclization procedure was followed using 2,5-dimethyl-3-nitromethyl-hexanoic acid methyl ester $(1.09 \mathrm{~g}, 5.02 \mathrm{mmol})$. Purification by column chromatography $(19: 1 \mathrm{EtOAc} / \mathrm{MeOH})$ gave 64 $(0.670 \mathrm{~g}, 86 \%, 84: 16 \mathrm{dr})$ as an oil. Major diastereomer: ${ }^{1} \mathbf{H} \mathbf{N M R}\left(\mathrm{CDCl}_{3}, 500 \mathrm{MHz}\right) \delta 0.86(\mathrm{~d}$, $\left.J=6.7 \mathrm{~Hz}, 3 \mathrm{H}, \mathrm{CH}_{2} \mathrm{CHMe}_{2}\right), 0.88\left(\mathrm{~d}, J=6.4 \mathrm{~Hz}, 3 \mathrm{H}, \mathrm{CH}_{2} \mathrm{CHMe}_{2}\right), 1.14(\mathrm{~d}, J=6.9 \mathrm{~Hz}, 3 \mathrm{H}$, CHMe), 1.28 (ddd, $J=13.39 .3,5.6 \mathrm{~Hz}, 1 \mathrm{H}, \mathrm{C}_{2} \mathrm{CHMe}_{2}$ ), 1.40 (ddd, $J=13.58 .5,4.7 \mathrm{~Hz}, 1 \mathrm{H}$, $\mathrm{C}_{2} \mathrm{CHMe}_{2}$ ), 1.55 (m, 1H, $\mathrm{CH}_{2} \mathrm{CHMe}_{2}$ ), 1.94-2.07 (m, 2H, $\underline{\mathrm{HMMe}}, \mathrm{NCH}_{2} \mathrm{C} \underline{\mathrm{H}}$ ), 2.87 (t, $J=9.2$ $\left.\mathrm{Hz}, 1 \mathrm{H}, \mathrm{NC}_{2}\right), 3.40\left(\mathrm{dd}, J=9.5,7.5 \mathrm{~Hz}, 1 \mathrm{H}, \mathrm{NC}_{2}\right), 7.08(\mathrm{br} \mathrm{m}, 1 \mathrm{H}, \mathrm{NH}) ;{ }^{13} \mathbf{C} \mathbf{~ N M R}\left(\mathrm{CDCl}_{3}\right.$, $125.6 \mathrm{MHz}) \delta 14.4\left(\mathrm{CH}_{3}\right), 21.9\left(\mathrm{CH}_{3}\right), 23.3\left(\mathrm{CH}_{3}\right), 25.8(\mathrm{CH}), 41.4(\mathrm{CH}), 42.5(\mathrm{CH}), 42.8$ $\left(\mathrm{CH}_{2}\right), 46.6\left(\mathrm{CH}_{2}\right), 181.1$ (C). Anal. Calcd for $\mathrm{C}_{9} \mathrm{H}_{17} \mathrm{NO}$ : C, 69.63; H, 11.04; N, 9.02. Found C, 69.33; H, 11.30; N, 8.97. 


\section{Conversion of 27 to 65.}

Part 1: 4-Nitro-3-phenyl-butyric Acid Methyl Ester. ${ }^{6}$ The general procedure for conversion to 4-nitro-butyric acid methyl esters was followed using 27 (2.90 g, $7.03 \mathrm{mmol}$ ). Purification by column chromatography (4:1 pet ether/EtOAc) gave the title compound (1.32 g, $84 \%)$ as an oil. ${ }^{1} \mathbf{H}$ NMR $\left(\mathrm{CDCl}_{3}, 500 \mathrm{MHz}\right) \delta 2.78\left(\mathrm{dd}, J=7.3,1.5 \mathrm{~Hz}, 2 \mathrm{H}, \mathrm{C}_{2} \mathrm{CO}_{2} \mathrm{Me}\right), 3.63$ (s, 3H, $\left.\mathrm{OC}_{3} \underline{3}\right), 3.99$ (quint. $J=7.5 \mathrm{~Hz}, 1 \mathrm{H}, \mathrm{C} \underline{\mathrm{HPh}}$ ), 4.64 (dd, $J=12.6,7.9 \mathrm{~Hz}, 1 \mathrm{H}, \mathrm{NO}_{2} \mathrm{CH}_{2}$ ), $4.74\left(\mathrm{dd}, J=12.9,7.1 \mathrm{~Hz}, 1 \mathrm{H}, \mathrm{NO}_{2} \mathrm{C}_{2}\right), 7.20-7.37(\mathrm{~m}, 5 \mathrm{H}, \mathrm{PhH}) ;{ }^{13} \mathbf{C ~ N M R}\left(\mathrm{CDCl}_{3}, 125.6\right.$ MHz) $\delta 37.4\left(\mathrm{CH}_{2}\right), 40.1(\mathrm{CH}), 51.9\left(\mathrm{CH}_{3}\right), 79.3\left(\mathrm{CH}_{2}\right), 127.3(\mathrm{CH}), 128.0(\mathrm{CH}), 129.1(\mathrm{CH})$, 138.2 (C), 171.1 (C). Anal. Calcd for $\mathrm{C}_{11} \mathrm{H}_{13} \mathrm{NO}_{4}$ : C, 59.19; H, 5.87; N, 6.27. Found C, 59.03; H, $5.59 ; \mathrm{N}, 6.30$.

Part 2: 4-Phenyl-pyrrolidin-2-one (65). The general hydrogenation-cyclization procedure was followed using 4-nitro-3-phenyl-butyric acid methyl ester (1.28 g, $5.73 \mathrm{mmol})$. Purification by column chromatography $\left(19: 1 \mathrm{CH}_{2} \mathrm{Cl}_{2} / \mathrm{MeOH}\right)$ gave $\mathbf{6 5}(0.828 \mathrm{~g}, 90 \%$, ) as a white solid: mp 74-77 ${ }^{\circ} \mathrm{C}$. lit. ${ }^{6} \mathrm{mp}$ 91-93 ${ }^{\circ} \mathrm{C}$ (enantiomer). The ${ }^{1} \mathrm{H}$ NMR and ${ }^{13} \mathrm{C}$ NMR spectra

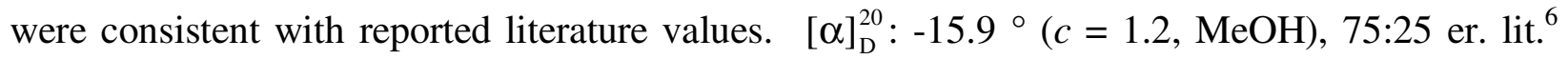
(enantiomer) $[\alpha]_{\mathrm{D}}^{20}:-33.8^{\circ}(c=0.89, \mathrm{MeOH})$.

3,4-Diphenyl-pyrrolidine-1-carboxylic Acid tert-Butyl Ester (66). The general reduction and protection procedure was followed using $62(0.055 \mathrm{~g}, 0.234 \mathrm{mmol})$. Purification by column chromatography (10:1 pet ether/EtOAc) gave $66(0.044 \mathrm{~g}, 58 \%$,) as a white solid: mp 117-119 ${ }^{\circ} \mathrm{C} .{ }^{1} \mathbf{H}$ NMR $\left(\mathrm{CDCl}_{3}, 500 \mathrm{MHz}\right) \delta 1.50\left(\mathrm{~s}, 9 \mathrm{H}, \mathrm{C}\left(\mathrm{CH}_{3}\right)_{3}\right), 3.50\left(\mathrm{~m}, 4 \mathrm{H}, \mathrm{CHPh}, \mathrm{NCH}_{2}\right)$, $3.95\left(\mathrm{~m}, 1 \mathrm{H}, \mathrm{NCH}_{2}\right), 4.04\left(\mathrm{~m}, 1 \mathrm{H}, \mathrm{NCH}_{2}\right), 7.13-7.30(\mathrm{~m}, 10 \mathrm{H}, \mathrm{PhH}) ;{ }^{13} \mathbf{C ~ N M R}\left(\mathrm{CDCl}_{3}, 125.6\right.$ MHz) $\delta 28.5\left(\mathrm{CH}_{3}\right), 50.4(\mathrm{CH}), 51.5(\mathrm{CH}), 52.8\left(\mathrm{CH}_{2}\right), 53.5\left(\mathrm{CH}_{2}\right), 79.4(\mathrm{C}), 126.9(\mathrm{CH}), 127.0$ (CH), $127.5(\mathrm{CH}), 128.5(\mathrm{CH}), 139.3(\mathrm{C}), 154.3(\mathrm{C})$. Anal. Calcd for $\mathrm{C}_{21} \mathrm{H}_{25} \mathrm{NO}_{2}$ : C, 77.98; H, 7.79; N, 4.33. Found C, 77.70; H, 7.91; N, 4.38. $[\alpha]_{\mathrm{D}}^{20}:-91.8^{\circ}\left(c=1.0, \mathrm{CHCl}_{3}\right)$.

3-Methyl-4-phenyl-pyrrolidine-1-carboxylic Acid tert-Butyl Ester (67). The general reduction and protection procedure was followed using $63(0.230 \mathrm{~g}, 1.31 \mathrm{mmol})$. Purification by column chromatography (8:1 pet ether/EtOAc) gave $67\left(0.280 \mathrm{~g}, 82 \%\right.$,) as an oil. ${ }^{1} \mathbf{H}$ NMR 
$\left(\mathrm{CDCl}_{3}\right.$, rotational isomers, $\left.500 \mathrm{MHz}\right) \delta 0.95\left(\mathrm{~d}, J=8.1 \mathrm{~Hz}, 3 \mathrm{H}, \mathrm{CHC}_{\underline{3}}\right), 1.47(\mathrm{~s}, 9 \mathrm{H}, \mathrm{Ot}-\mathrm{Bu})$,

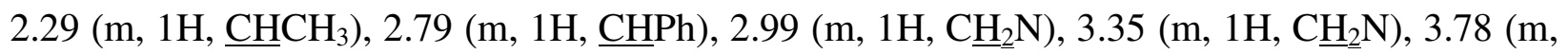
$\left.2 \mathrm{H}, \mathrm{C}_{2} \mathrm{~N}\right), 7.23(\mathrm{~m}, 3 \mathrm{H}, \mathrm{Ph} \underline{\mathrm{H}}), 7.32(\mathrm{~m}, 2 \mathrm{H}, \mathrm{Ph} \underline{\mathrm{H}}) ;{ }^{13} \mathbf{C} \mathbf{~ N M R}\left(\mathrm{CDCl}_{3}\right.$, rotational isomers, 125.6 MHz) $\delta 15.4\left(\mathrm{CH}_{3}\right), 27.4\left(\mathrm{CH}_{3}\right), 39.8(\mathrm{CH}), 40.8(\mathrm{CH}), 51.6(\mathrm{CH}), 52.4(\mathrm{CH}), 52.8\left(\mathrm{CH}_{2}\right), 52.9$ $\left(\mathrm{CH}_{2}\right), 53.4\left(\mathrm{CH}_{2}\right), 53.7\left(\mathrm{CH}_{2}\right), 79.0(\mathrm{C}), 126.8(\mathrm{CH}), 126.9(\mathrm{CH}), 127.6(\mathrm{CH}), 128.5(\mathrm{CH}), 139.8$ (C), 139.9 (C), 154.2 (C), 154.3 (C). Anal. Calcd for $\mathrm{C}_{16} \mathrm{H}_{23} \mathrm{NO}_{2}$ : C, 73.53; H, 8.87; N, 5.36. Found C, 73.56; H, 9.06; N, 5.05. $[\alpha]_{\mathrm{D}}^{20}:-26.4^{\circ}\left(c=1.1, \mathrm{CHCl}_{3}\right)$.

3-iso-Butyl-4-methyl-pyrrolidine-1-carboxylic Acid tert-Butyl Ester (68). The general reduction and protection procedure was followed using $64(0.120 \mathrm{~g}, 0.773 \mathrm{mmol})$. Purification by column chromatography (10:1 pet ether/EtOAc) gave $\mathbf{6 8}\left(0.148 \mathrm{~g}, 79 \%\right.$, ) as an oil. ${ }^{1} \mathbf{H}$ NMR $\left(\mathrm{CDCl}_{3}, 500 \mathrm{MHz}\right) \delta 0.86\left(\mathrm{~d}, J=6.6 \mathrm{~Hz}, 3 \mathrm{H}, \mathrm{CH}_{2} \mathrm{CHMe}_{2}\right), 0.88\left(\mathrm{~d}, J=6.4 \mathrm{~Hz}, 3 \mathrm{H}, \mathrm{CH}_{2} \mathrm{CHMe}_{2}\right)$, 0.97 (d, $J=6.2 \mathrm{~Hz}, 3 \mathrm{H}, \mathrm{CHMe}$ ), 1.09 (ddd, $J=13.5,10.0,4.7 \mathrm{~Hz}, 1 \mathrm{H}, \mathrm{C}_{2} \mathrm{CHMe}_{2}$ ), 1.33 (ddd, $J$ $\left.=13.3,9.4,3.7 \mathrm{~Hz}, 1 \mathrm{H}, \mathrm{C}_{2} \mathrm{CHMe}_{2}\right), 1.43\left(\mathrm{~s}, 9 \mathrm{H}, \mathrm{C}\left(\mathrm{CH}_{3}\right)_{3}\right), 1.52\left(\mathrm{~m}, 1 \mathrm{H}, \mathrm{CH}_{2} \mathrm{CHMe}_{2}\right), 1.67(\mathrm{~m}$, $\left.2 \mathrm{H}, \mathrm{NCH}_{2} \mathrm{C} \underline{\mathrm{H}}, \mathrm{C} \underline{\mathrm{HMe}}\right), 2.81\left(\mathrm{~m}, 2 \mathrm{H}, \mathrm{NC}_{2}\right), 3.53\left(\mathrm{dd}, J=10.8,7.5 \mathrm{~Hz}, 1 \mathrm{H}, \mathrm{NC}_{2}\right), 3.58$ (dd, $\left.J=10.8,7.3 \mathrm{~Hz}, 1 \mathrm{H}, \mathrm{NCH}_{2}\right) ;{ }^{13} \mathbf{C} \mathbf{~ N M R}\left(\mathrm{CDCl}_{3}, 125.6 \mathrm{MHz}\right) \delta 15.8\left(\mathrm{CH}_{3}\right), 21.7\left(\mathrm{CH}_{3}\right), 23.9$ $\left(\mathrm{CH}_{3}\right), 26.7(\mathrm{CH}), 28.5\left(\mathrm{CH}_{3}\right), 39.1(\mathrm{CH}), 41.4\left(\mathrm{CH}_{2}\right), 43.4(\mathrm{CH}), 51.9\left(\mathrm{CH}_{2}\right), 53.1\left(\mathrm{CH}_{2}\right), 78.8$ (C), 154.4 (C). HRMS-FAB (M+1) Calcd for $\mathrm{C}_{14} \mathrm{H}_{28} \mathrm{NO}_{2}$ : 242.2120; Found: 242.2120. $[\alpha]_{\mathrm{D}}^{20}$ : $87.5^{\circ}\left(c=0.80, \mathrm{CHCl}_{3}\right)$.

3-Phenyl-pyrrolidine-1-carboxylic Acid tert-Butyl Ester (69). The general reduction and protection procedure was followed using $65(0.150 \mathrm{~g}, 0.930 \mathrm{mmol})$. Purification by column chromatography (10:1 pet ether/EtOAc) gave $69\left(0.181 \mathrm{~g}, 79 \%\right.$,) as an oil. The ${ }^{1} \mathrm{H}$ NMR and ${ }^{13} \mathrm{C}$ NMR spectra were consistent with reported literature values. ${ }^{7}[\alpha]_{\mathrm{D}}^{20}:+5.5^{\circ}\left(c=1.6, \mathrm{CHCl}_{3}\right)$.

\section{\{3-[tert-Butoxycarbonyl-(4-methoxy-phenyl)-amino]-2,3-diphenyl-propyl\}-carbamic} Acid tert-Butyl Ester (70). A mixture of 36 (0.200 g, $0.430 \mathrm{mmol})$ and Raney nickel (1 mL) in $\mathrm{MeOH}(10 \mathrm{~mL})$ was stirred under $200 \mathrm{psi}$ of $\mathrm{H}_{2}$. After $48 \mathrm{~h}$, the solution was filtered through celite pad and extracted with ether and $\mathrm{CH}_{2} \mathrm{Cl}_{2}$. After evaporation of the solvent, the residue was dissolved in $\mathrm{CH}_{2} \mathrm{Cl}_{2}(10 \mathrm{~mL})$ and treated with $\mathrm{Boc}_{2} \mathrm{O}(0.090 \mathrm{~g}, 0.430 \mathrm{mmol})$ for $6 \mathrm{~h}$ at room 
temperature. After evaporation of the solvent, the residue was separated with flash chromatography on silica gel (9:1 hexanes/EtOAc) affording 70 (0.170 g, 74\%): mp 114-115 ${ }^{\circ} \mathrm{C}$; ${ }^{1}$ H NMR $\left(500 \mathrm{MHz}, \mathrm{CDCl}_{3}\right) \delta 1.35(\mathrm{~s}, 9 \mathrm{H}), 1.42(\mathrm{~s}, 9 \mathrm{H}), 3.30-3.56(\mathrm{~m}, 2 \mathrm{H}), 3.78(\mathrm{~s}, 3 \mathrm{H}), 4.36-$ $4.28(\mathrm{~m}, 1 \mathrm{H}), 4.78(\mathrm{~s}, 1 \mathrm{H}), 6.05(\mathrm{~s}, 1 \mathrm{H}), 6.71(\mathrm{~s}, 3 \mathrm{H}), 6.86(\mathrm{~d}, J=6.0 \mathrm{~Hz}, 2 \mathrm{H}), 6.72-7.20(\mathrm{~m}$, 9H); ${ }^{13} \mathrm{C}$ NMR $\left(125.7 \mathrm{MHz}, \mathrm{CDCl}_{3}\right) \delta 28.2,28.4,44.3,47.0,55.2,61.5,78.8,80.1,126.4,127.0$, $127.5,128.3,128.7,129.2,129.8,131.2,137.9,139.9,155.8,156.3,158.2$. Anal. Calcd for $\mathrm{C}_{32} \mathrm{H}_{40} \mathrm{~N}_{2} \mathrm{O}_{5}$ : C, 72.15; H, 7.57; N, 5.26. Found: C, 71.43; H, 7.53; N, 5.31.

1-(4-Methoxy-phenyl)-4,5-diphenyl-tetrahydro-pyrimidin-2-one (71). A solution of $70(0.100 \mathrm{~g}, 0.186 \mathrm{mmol})$ and $\mathrm{KO} t \mathrm{Bu}(0.040 \mathrm{~g}, 0.372 \mathrm{mmol})$ in THF $(5 \mathrm{~mL})$ was heated at reflux for $1 \mathrm{~h}$. The reaction mixture was diluted with ether, washed with $\mathrm{H}_{2} \mathrm{O}$, and dried over anhydrous $\mathrm{MgSO}_{4}$. After evaporation of the solvent, the residue was separated by flash column chromatography (EtOAc) affording 71 (0.060 g, $90 \%)$ : mp 228-229 ${ }^{\circ} \mathrm{C} ;{ }^{1} \mathbf{H}$ NMR (500 MHz, $\left.\mathrm{CDCl}_{3}\right) \delta 3.25-3.33(\mathrm{~m}, 1 \mathrm{H}), 3.55-3.63(\mathrm{~m}, 2 \mathrm{H}), 3.66(\mathrm{~s}, 3 \mathrm{H}), 4.92(\mathrm{~d}, J=3.5 \mathrm{~Hz}, 1 \mathrm{H}), 5.37(\mathrm{~s}$, $1 \mathrm{H}), 6.68(\mathrm{~d}, J=9.0 \mathrm{~Hz}, 2 \mathrm{H}), 6.86(\mathrm{~d}, J=9.0 \mathrm{~Hz}, 2 \mathrm{H}), 7.15-7.50(\mathrm{~m}, 10 \mathrm{H}) ;{ }^{13} \mathbf{C}$ NMR $(125.7$ $\left.\mathrm{MHz}, \mathrm{CDCl}_{3}\right) \delta 41.7,44.3,55.2,69.3,113.8,126.5,127.3,127.5,127.7,128.2,128.7,128.9$, 135.6, 141.3, 141.4, 156.0, 157.6. HRMS Calcd for $\mathrm{C}_{23} \mathrm{H}_{22} \mathrm{~N}_{2} \mathrm{O}_{2}$ : 358.1681; Found: 358.1681.

1-Benzyl-4-phenyl-5-triisopropylsilanyloxy-piperidin-2-one (74). The general benzylation procedure was followed using $57(0.400 \mathrm{~g}, 1.15 \mathrm{mmol})$. Purification by column chromatography (3:1 pet ether/EtOAc) gave $(0.451 \mathrm{~g}, 90 \%)$ as a colorless oil: ${ }^{1} \mathbf{H} \mathbf{~ N M R}\left(\mathrm{CDCl}_{3}\right.$, $\left.500 \mathrm{MHz}) \delta 0.98\left(\mathrm{~m}, 21 \mathrm{H},\left(\left(\mathrm{C}_{3}\right)_{2}\right)_{2} \underline{\mathrm{H}}\right)_{3} \mathrm{Si}\right), 2.75\left(\mathrm{dd}, J=17.6,3.6 \mathrm{~Hz}, 1 \mathrm{H}, \mathrm{CHC}_{2} \underline{\mathrm{H}}_{2} \mathrm{CON}\right), 3.06$ (m, 2H, $\mathrm{NC}_{2}$ ), 3.14 (dd, $\left.J=17.8,6.6 \mathrm{~Hz}, 1 \mathrm{H}, \mathrm{CHCH}_{2} \mathrm{CON}\right), 3.32$ (q, $J=5.4 \mathrm{~Hz}, 1 \mathrm{H}, \mathrm{CHPh}$ ), 4.13 (q, $J=3.4 \mathrm{~Hz}, 1 \mathrm{H}, \mathrm{C} \underline{\mathrm{HOTIPS}}), 4.51$ (d, $\left.J=14.7 \mathrm{~Hz}, 1 \mathrm{H}, \mathrm{NC}_{2} \underline{\mathrm{Ph}}\right), 4.71$ (d, $J=15.0 \mathrm{~Hz}$, $\left.1 \mathrm{H}, \mathrm{NC}_{2} \underline{\mathrm{Ph}}\right), 7.17(\mathrm{~m}, 2 \mathrm{H}, \mathrm{Ph} \underline{\mathrm{H}}), 7.26(\mathrm{~m}, 8 \mathrm{H}, \mathrm{Ph} \underline{\mathrm{H}}) ;{ }^{13} \mathbf{C} \mathbf{N M R}\left(\mathrm{CDCl}_{3}, 125.6 \mathrm{MHz}\right) \delta 12.1$ $(\mathrm{CH}), 17.9\left(\mathrm{CH}_{3}\right), 32.6\left(\mathrm{CH}_{2}\right), 45.3(\mathrm{CH}), 49.8\left(\mathrm{CH}_{2}\right), 50.8\left(\mathrm{CH}_{2}\right), 69.9(\mathrm{CH}), 127.0(\mathrm{CH}), 127.2$ $(\mathrm{CH}), 127.3(\mathrm{CH}), 128.1(\mathrm{CH}), 128.4(\mathrm{CH}), 128.7(\mathrm{CH}), 136.6(\mathrm{C}), 140.8(\mathrm{C}), 169.0(\mathrm{C})$. HRMSFAB (M+1) Calcd for $\mathrm{C}_{27} \mathrm{H}_{40} \mathrm{NO}_{2} \mathrm{Si}$ : 438.2828; Found: 438.2826 . 


\section{Conversion of 74 to 78.}

Part 1: 3-Allyl-1-benzyl-4-phenyl-5-triisopropylsilanyloxy-piperidin-2-one. The general procedure for $t$-BuLi enolization was followed using $74(0.235 \mathrm{~g}, 0.537 \mathrm{mmol})$ and allyl bromide $(0.185 \mathrm{~mL}, 2.14 \mathrm{mmol})$. Purfication by column chromatography (5:1 pet ether/EtOAc) gave the title compound $(0.227 \mathrm{~g}, 89 \%,>97: 3 \mathrm{dr})$ as a colorless oil. ${ }^{\mathbf{1}} \mathbf{H} \mathbf{~ N M R}\left(\mathrm{CDCl}_{3}, 400\right.$ $\mathrm{MHz}) \delta 0.67\left(\mathrm{~m}, 3 \mathrm{H}, \mathrm{CH}_{3} \mathrm{C} \underline{\mathrm{HSi}}\right), 0.75\left(\mathrm{~d}, J=6.8 \mathrm{~Hz}, 9 \mathrm{H}, \mathrm{C}_{3} \underline{\mathrm{CHSi}}\right), 0.79$ (d, $J=6.8 \mathrm{~Hz}, 9 \mathrm{H}$, $\mathrm{CH}_{3}{ }_{2} \mathrm{CHSi}$ ), 2.12 (quint, $J=6.1 \mathrm{~Hz}, 1 \mathrm{H}, \mathrm{CH}_{2}=\mathrm{CHCH}_{2} \mathrm{CH}$ ), $2.82\left(\mathrm{~m}, 2 \mathrm{H}, \mathrm{CH}_{2}=\mathrm{CHCH}_{2} \mathrm{CH}\right.$ ), 2.98 (t, $J=9.3 \mathrm{~Hz}, 1 \mathrm{H}, \mathrm{C} \underline{H P h}), 3.17\left(\mathrm{dd}, J=11.9,8.5 \mathrm{~Hz}, 1 \mathrm{H}, \mathrm{NC}_{2}\right), 3.32(\mathrm{dd}, J=11.9,4.6 \mathrm{~Hz}, 1 \mathrm{H}$, $\left.\mathrm{NC}_{2}{ }_{2}\right), 4.09$ (td, $\left.J=8.8,4.6 \mathrm{~Hz}, 1 \mathrm{H}, \mathrm{C} \underline{\mathrm{HOTIPS}}\right), 4.50\left(\mathrm{~d}, J=14.4 \mathrm{~Hz}, 1 \mathrm{H}, \mathrm{NC}_{2} \mathrm{Ph}\right), 4.80(\mathrm{~d}, J$ $\left.=14.4 \mathrm{~Hz}, 1 \mathrm{H}, \mathrm{PhCH}_{2} \mathrm{~N}\right), 5.05\left(\mathrm{~m}, 2 \mathrm{H}, \mathrm{CH}_{2}=\mathrm{CH}\right), 5.74\left(\mathrm{~m}, 1 \mathrm{H}, \mathrm{CH}_{2}=\mathrm{CHCH}_{2}\right), 7.15-7.23(\mathrm{~m}$, 3H, Ph프), $7.30(\mathrm{~m}, 7 \mathrm{H}, \mathrm{Ph} \underline{\mathrm{H}}) ;{ }^{13} \mathrm{C}$ NMR $\left(\mathrm{CDCl}_{3}, 100.6 \mathrm{MHz}\right) \delta 12.3(\mathrm{CH}), 17.7\left(\mathrm{CH}_{3}\right), 17.7$ $\left(\mathrm{CH}_{3}\right), 34.1\left(\mathrm{CH}_{2}\right), 45.9(\mathrm{CH}), 50.3\left(\mathrm{CH}_{2}\right), 51.2(\mathrm{CH}), 52.9\left(\mathrm{CH}_{2}\right), 70.5(\mathrm{CH}), 117.8\left(\mathrm{CH}_{2}\right), 126.9$ $(\mathrm{CH}), 127.5(\mathrm{CH}), 128.3(\mathrm{CH}), 128.4(\mathrm{CH}), 128.4(\mathrm{CH}), 128.6(\mathrm{CH}), 135.1(\mathrm{CH}), 136.9(\mathrm{C})$, 141.0 (C), 170.7 (C). HRMS-FAB (M+1) Calcd for $\mathrm{C}_{30} \mathrm{H}_{44} \mathrm{NO}_{2} \mathrm{Si}$ : 478.3141; Found: 478.3140. $[\alpha]_{\mathrm{D}}^{20}:-56.3^{\circ}\left(c=2.2, \mathrm{CHCl}_{3}\right)$.

Part 2: 3-Allyl-1-benzyl-4-phenyl-5-triisopropylsilanyloxy-piperidine (78). The general reduction procedure for benzyl lactams was followed using 3-allyl-1-benzyl-4-phenyl-5triisopropylsilanyloxy-piperidin-2-one $(0.112 \mathrm{~g}, 0.236 \mathrm{mmol})$ and lithium aluminum hydride $(0.708 \mathrm{~mL}$ of a $1 \mathrm{M}$ solution in THF, $0.708 \mathrm{mmol})$ with a $6 \mathrm{~h}$ reflux period. Purification by column chromatography (15:1 pet ether/EtOAc) provided $78(0.094 \mathrm{~g}, 86 \%)$ as a colorless oil. ${ }^{1} \mathbf{H}$ NMR $\left(\mathrm{CDCl}_{3}, 500 \mathrm{MHz}\right) \delta 0.67\left(\mathrm{~m}, 3 \mathrm{H}, \mathrm{CH}_{3} \mathrm{CHSi}\right), 0.75\left(\mathrm{~d}, J=6.8 \mathrm{~Hz}, 9 \mathrm{H}, \mathrm{CH}_{3} \underline{\mathrm{CHSi}}\right), 0.79$ $\left(\mathrm{d}, J=6.8 \mathrm{~Hz}, 9 \mathrm{H}, \mathrm{C}_{\underline{3}} \mathrm{CHSi}\right), 1.68\left(\mathrm{dt}, J=14.4,8.8 \mathrm{~Hz}, 1 \mathrm{H}, \mathrm{C}_{2}{ }_{2} \mathrm{~N}\right), 1.90(\mathrm{~m}, 3 \mathrm{H}$, $\left.\mathrm{CH}_{2}=\mathrm{CHCH}_{2} \underline{\mathrm{CH}}_{2} \mathrm{CH}_{2} \mathrm{~N}\right), 2.07\left(\mathrm{~m}, 1 \mathrm{H}, \mathrm{CH}_{2}=\mathrm{CHCH}_{2} \mathrm{CH}\right), 2.18(\mathrm{dd}, J=11.6,9.6 \mathrm{~Hz}, 1 \mathrm{H}$, C $\underline{H P h}), 3.06\left(\mathrm{ddd}, J=11.2,3.9,1.3 \mathrm{~Hz}, 1 \mathrm{H}, \mathrm{NCH}_{2}\right), 3.11(\mathrm{ddd}, J=11.7,4.5,1.5 \mathrm{~Hz}, 1 \mathrm{H}$, $\left.\mathrm{NC}_{2}\right), 3.48\left(\mathrm{~d}, J=13.1 \mathrm{~Hz}, 1 \mathrm{H}, \mathrm{NC}_{2} \underline{\mathrm{H}}_{2} \mathrm{Ph}\right), 3.76\left(\mathrm{~d}, J=12.9 \mathrm{~Hz}, 1 \mathrm{H}, \mathrm{PhC}_{2} \underline{\mathrm{N}}_{2}\right), 3.97(\mathrm{td}, J=$ 9.6, $4.5 \mathrm{~Hz}, 1 \mathrm{H}, \mathrm{CHOTIPS}), 4.90\left(\mathrm{~m}, 2 \mathrm{H}, \mathrm{CH}_{2}=\mathrm{CH}\right), 5.65\left(\mathrm{~m}, 1 \mathrm{H}, \mathrm{CH}_{2}=\mathrm{CHCH}_{2}\right), 7.18-7.31$ (m, 6H, PhH $), 7.36(\mathrm{~m}, 4 \mathrm{H}, \mathrm{Ph} \underline{\mathrm{H}}) ;{ }^{13} \mathrm{C} \mathbf{N M R}\left(\mathrm{CDCl}_{3}, 125.6 \mathrm{MHz}\right) \delta 12.4(\mathrm{CH}), 17.8\left(\mathrm{CH}_{3}\right), 17.8$ $\left(\mathrm{CH}_{3}\right), 35.9\left(\mathrm{CH}_{2}\right), 39.8(\mathrm{CH}), 57.2(\mathrm{CH}), 58.9\left(\mathrm{CH}_{2}\right), 61.2\left(\mathrm{CH}_{2}\right), 62.8\left(\mathrm{CH}_{2}\right), 73.7(\mathrm{CH}), 116.0$ $\left(\mathrm{CH}_{2}\right), 126.3(\mathrm{CH}), 127.0(\mathrm{CH}), 128.0(\mathrm{CH}), 128.1(\mathrm{CH}), 129.2(\mathrm{CH}), 136.4(\mathrm{CH}), 137.9(\mathrm{C})$, 
142.1 (C). HRMS-FAB (M-1) Calcd for $\mathrm{C}_{30} \mathrm{H}_{44} \mathrm{NOSi}$ : 462.3192; Found: 462.3192. [ $\left.\alpha\right]_{\mathrm{D}}^{20}:-32.7^{\circ}$ $\left(c=1.3, \mathrm{CHCl}_{3}\right)$.

2-Oxo-3,4-diphenyl-pyrrolidine-1-carboxylic Acid tert-Butyl Ester (79). The general procedure for the protection of pyrrolidinones was followed using $62(0.100 \mathrm{~g}, 0.421 \mathrm{mmol})$. Purification by column chromatography (4:1 pet ether/EtOAc) provided $79(0.110 \mathrm{~g}, 77 \%)$ as a colorless oil. ${ }^{1} \mathbf{H}$ NMR $\left(\mathrm{CDCl}_{3}, 500 \mathrm{MHz}\right) \delta 1.59(\mathrm{~s}, 9 \mathrm{H}, \mathrm{Ot}-\mathrm{Bu}), 3.63(\mathrm{td}, J=11.0,8.1 \mathrm{~Hz}, 1 \mathrm{H}$, CㅍPh), 3.79 (t, $J=11.3 \mathrm{~Hz}, 1 \mathrm{H}, \mathrm{CH}_{2} \mathrm{~N}$ ), $3.94(\mathrm{~d}, J=11.8 \mathrm{~Hz}, 1 \mathrm{H}, \mathrm{COC} \underline{\mathrm{HPh}}$ ), 4.26 (dd, $J=10.7$, $\left.8.1 \mathrm{~Hz}, 1 \mathrm{H}, \mathrm{C}_{2} \mathrm{~N}\right), 7.15-7.36(\mathrm{~m}, 10 \mathrm{H}, \mathrm{Ph} \underline{\mathrm{H}}) ;{ }^{13} \mathbf{C} \mathbf{N M R}\left(\mathrm{CDCl}_{3}, 125.6 \mathrm{MHz}\right) \delta 27.9\left(\mathrm{CH}_{3}\right)$, $45.7(\mathrm{CH}), 50.9\left(\mathrm{CH}_{2}\right), 57.0(\mathrm{CH}), 83.1(\mathrm{C}), 127.2(\mathrm{CH}), 127.4(\mathrm{CH}), 127.5(\mathrm{CH}), 128.5(\mathrm{CH})$, $128.5(\mathrm{CH}), 128.8(\mathrm{CH}), 136.0(\mathrm{C}), 138.4(\mathrm{C}), 150.0(\mathrm{C}), 172.7$ (C). HRMS-EI (M+) Calcd for $\mathrm{C}_{21} \mathrm{H}_{23} \mathrm{NO}_{3}$ : 337.1678; Found: $337.1684 .[\alpha]_{\mathrm{D}}^{20}:-87.0^{\circ}\left(c=1.1, \mathrm{CHCl}_{3}\right)$

3-Methyl-2-oxo-4-phenyl-pyrrolidine-1-carboxylic Acid tert-Butyl Ester (80). The general procedure for the protection of pyrrolidinones was followed using $64(0.098 \mathrm{~g}, 0.559$ mmol). Purification by column chromatography (4:1 pet ether/EtOAc) provided $\mathbf{8 0}(0.148 \mathrm{~g}, 90$ $\%)$ as a white solid: $\mathrm{mp} 107-108{ }^{\circ} \mathrm{C}^{\mathbf{1}} \mathbf{H} \mathbf{~ N M R}\left(\mathrm{CDCl}_{3}, 500 \mathrm{MHz}\right) \delta 1.20(\mathrm{~d}, J=6.8 \mathrm{~Hz}, 3 \mathrm{H}$, $\left.\mathrm{CHCH}_{3}\right), 1.55(\mathrm{~s}, 9 \mathrm{H}, \mathrm{Ot}-\underline{\mathrm{Bu}}), 2.71\left(\mathrm{dq}, J=13.5,6.9 \mathrm{~Hz}, 3 \mathrm{H}, \mathrm{CHCH}_{3}\right), 3.02(\mathrm{q}, J=10.7 \mathrm{~Hz}, 1 \mathrm{H}$, $\underline{\mathrm{CHPh}}$ ), $3.60\left(\mathrm{t}, J=10.9 \mathrm{~Hz}, 1 \mathrm{H}, \mathrm{C}_{2} \mathrm{~N}\right), 4.07\left(\mathrm{dd}, J=10.9,8.4 \mathrm{~Hz}, 1 \mathrm{H}, \underline{\mathrm{C}}_{2} \mathrm{~N}\right), 7.24-7.42(\mathrm{~m}$, 5H, PhH $) ;{ }^{13} \mathbf{C ~ N M R}\left(\mathrm{CDCl}_{3}, 125.6 \mathrm{MHz}\right) \delta 13.3\left(\mathrm{CH}_{3}\right), 27.9\left(\mathrm{CH}_{3}\right), 45.6(\mathrm{CH}), 45.9(\mathrm{CH}), 51.2$ $\left(\mathrm{CH}_{2}\right), 82.8(\mathrm{C}), 127.3(\mathrm{CH}), 127.5(\mathrm{CH}), 128.9(\mathrm{CH}), 138.8(\mathrm{C}), 150.0(\mathrm{C}), 175.1(\mathrm{C})$. Anal. Calcd for $\mathrm{C}_{16} \mathrm{H}_{21} \mathrm{NO}_{3}: \mathrm{C}, 69.79 ; \mathrm{H}, 7.69 ; \mathrm{N}, 5.09$. Found $\mathrm{C}, 69.40 ; \mathrm{H}, 7.91 ; \mathrm{N}, 5.19 .[\alpha]_{\mathrm{D}}^{20}:-44.5$ ${ }^{\circ}\left(c=0.33, \mathrm{CHCl}_{3}\right)$.

\section{3-Allyl-3-methyl-2-oxo-4-phenyl-pyrrolidine-1-carboxylic Acid tert-Butyl Ester (82).} The general procedure for LDA enolization was followed using $80(0.075 \mathrm{~g}, 0.272 \mathrm{mmol})$ and allyl bromide $(0.071 \mathrm{~mL}, 0.816 \mathrm{mmol})$. Purfication by column chromatography (9:1 pet ether/EtOAc) gave $82(0.071 \mathrm{~g}, 83 \%,>97: 3 \mathrm{dr})$ as a colorless oil. ${ }^{1} \mathbf{H} \mathbf{~ N M R}\left(\mathrm{CDCl}_{3}, 400 \mathrm{MHz}\right)$ $\delta 0.87\left(\mathrm{~s}, 3 \mathrm{H}, \mathrm{CC}_{\underline{3}}\right), 1.57(\mathrm{~s}, 9 \mathrm{H}, \mathrm{Ot}-\underline{\mathrm{Bu}}), 2.13\left(\mathrm{dd}, J=14.1,9.0 \mathrm{~Hz}, 1 \mathrm{H}, \mathrm{C}_{2} \mathrm{CH}_{2} \mathrm{CH}_{2}\right), 2.54$ (dd, $\left.J=14.1,4.9 \mathrm{~Hz}, 1 \mathrm{H}, \mathrm{C}_{2} \underline{\mathrm{CH}}=\mathrm{CH}_{2}\right), 3.45(\mathrm{t}, J=10.7 \mathrm{~Hz}, 1 \mathrm{H}, \underline{\mathrm{CHPh}}), 3.94\left(\mathrm{~m}, 2 \mathrm{H}, \mathrm{C}_{2} \mathrm{~N}\right)$, 
$5.21\left(\mathrm{~d}, J=5.6 \mathrm{~Hz}, 1 \mathrm{H}, \mathrm{CH}_{2} \mathrm{CH}=\mathrm{C}_{2}\right), 5.24\left(\mathrm{~s}, 1 \mathrm{H}, \mathrm{CH}_{2} \mathrm{CH}=\mathrm{C}_{2}\right), 5.84\left(\mathrm{~m}, 1 \mathrm{H}, \mathrm{CH}_{2} \mathrm{CH}=\mathrm{CH}_{2}\right)$, $7.21(\mathrm{~m}, 2 \mathrm{H}, \mathrm{Ph} \underline{\mathrm{H}}), 7.33(\mathrm{~m}, 3 \mathrm{H}, \mathrm{Ph} \underline{\mathrm{H}}) ;{ }^{13} \mathbf{C} \mathbf{~ N M R}\left(\mathrm{CDCl}_{3}, 100.6 \mathrm{MHz}\right) \delta 19.4\left(\mathrm{CH}_{3}\right), 28.0$ $\left(\mathrm{CH}_{3}\right), 40.5\left(\mathrm{CH}_{2}\right), 43.1(\mathrm{CH}), 47.5\left(\mathrm{CH}_{2}\right), 49.9(\mathrm{C}), 83.0(\mathrm{C}), 119.2\left(\mathrm{CH}_{2}\right), 127.4(\mathrm{CH}), 128.4$ $(\mathrm{CH}), 128.6(\mathrm{CH}), 134.0(\mathrm{CH}), 136.7(\mathrm{C}), 150.1(\mathrm{C}), 177.0(\mathrm{C})$. HRMS-EI (M+) Calcd for $\mathrm{C}_{19} \mathrm{H}_{25} \mathrm{NO}_{3}$ : 315.1834; Found: 315.1825 .

3-Allyl-1,3-dimethyl-4-phenyl-pyrrolidine (83). The general reduction procedure for benzyl lactams was followed using $82(0.035 \mathrm{~g}, 0.111 \mathrm{mmol})$ and lithium aluminum hydride $(0.665 \mathrm{~mL}$ of a $1 \mathrm{M}$ solution in THF, $0665 \mathrm{mmol})$ with a $6 \mathrm{~h}$ reflux period. Purification by column chromatography on basic alumina (25:1 pet ether/EtOAc) provided $\mathbf{8 3}(0.017 \mathrm{~g}, 72 \%)$ as a colorless oil. ${ }^{1} \mathbf{H}$ NMR $\left(\mathrm{CDCl}_{3}, 500 \mathrm{MHz}\right) \delta 0.65\left(\mathrm{~s}, 3 \mathrm{H}, \mathrm{CCH}_{3}\right), 2.25(\mathrm{dd}, J=7.5,1.1 \mathrm{~Hz}, 1 \mathrm{H}$, $\mathrm{C} \underline{\mathrm{HPh}}$ ), 2.27 (dd, $\left.J=12.4,9.3 \mathrm{~Hz}, 2 \mathrm{H}, \underline{\mathrm{C}}_{2} \mathrm{CH}=\mathrm{CH}_{2}\right), 2.40\left(\mathrm{~s}, 3 \mathrm{H}, \mathrm{NC}_{\underline{3}}\right), 2.80(\mathrm{~d}, J=9.5 \mathrm{~Hz}$, $\left.1 \mathrm{H}, \underline{\mathrm{C}}_{2} \mathrm{~N}\right), 2.87\left(\mathrm{t}, J=9.2 \mathrm{~Hz}, 1 \mathrm{H}, \mathrm{C}_{2}{ }_{2} \mathrm{~N}\right), 3.00\left(\mathrm{t}, J=7.4 \mathrm{~Hz}, 1 \mathrm{H}, \underline{\mathrm{C}}_{2} \mathrm{~N}\right), 3.19$ (t, $J=8.6 \mathrm{~Hz}$, $\left.1 \mathrm{H}, \mathrm{C}_{2} \underline{\mathrm{N}}\right), 5.08\left(\mathrm{~m}, 1 \mathrm{H}, \mathrm{CH}_{2} \mathrm{CH}=\mathrm{C}_{2}\right), 5.11\left(\mathrm{~m}, 1 \mathrm{H}, \mathrm{CH}_{2} \mathrm{CH}=\mathrm{CH}_{2}\right), 5.24(\mathrm{~s}, 1 \mathrm{H}$, $\left.\mathrm{CH}_{2} \mathrm{CH}=\mathrm{C}_{2}\right), 5.85\left(\mathrm{~m}, 1 \mathrm{H}, \mathrm{CH}_{2} \mathrm{C} \underline{\mathrm{H}}=\mathrm{CH}_{2}\right), 7.18-7.31(\mathrm{~m}, 5 \mathrm{H}, \mathrm{Ph} \underline{\mathrm{H}}) ;{ }^{13} \mathbf{C} \mathbf{N M R}\left(\mathrm{CDCl}_{3}, 125.6\right.$ MHz) $\delta 23.5\left(\mathrm{CH}_{3}\right), 29.6\left(\mathrm{CH}_{2}\right), 42.8(\mathrm{CH}), 45.0(\mathrm{C}), 46.7\left(\mathrm{CH}_{2}\right), 53.3\left(\mathrm{CH}_{3}\right), 61.7\left(\mathrm{CH}_{2}\right), 69.1$ $\left(\mathrm{CH}_{2}\right), 117.5\left(\mathrm{CH}_{2}\right), 119.2\left(\mathrm{CH}_{2}\right), 126.2(\mathrm{CH}), 127.9(\mathrm{CH}), 128.9(\mathrm{CH}), 135.6(\mathrm{CH}), 140.6(\mathrm{C})$. HRMS-FAB (M+1) Calcd for $\mathrm{C}_{15} \mathrm{H}_{22} \mathrm{~N}$ : 216.1752; Found: 216.1751 .

2-Formyl-4,5-diphenyl-piperidine-1-carboxylic Acid tert-Butyl Ester (85). The general lithiation procedure was followed using $\mathbf{5 9}(0.100 \mathrm{~g}, 0.296 \mathrm{mmol})$ and DMF $(0.073 \mathrm{~mL}$, $0.946 \mathrm{mmol})$ as the electrophile. Purification by column chromatography (4:1 pet ether/EtOAc) gave 85 (0.079 g, $73 \%, 81: 19 \mathrm{dr})$ as an oil. Major diastereomer: ${ }^{1} \mathbf{H} \mathbf{~ N M R}\left(\mathrm{CDCl}_{3}, 500 \mathrm{MHz}\right)$ $\delta 1.50(\mathrm{~s}, 9 \mathrm{H}, \mathrm{Ot}-\underline{\mathrm{Bu}}), 1.83\left(\mathrm{q}, J=12.2 \mathrm{~Hz}, 1 \mathrm{H}, \mathrm{CHC}_{2} \mathrm{CH}\right), 2.13\left(\mathrm{~m} \mathrm{1H}, \mathrm{CHC}_{2} \mathrm{CH}\right), 3.05(\mathrm{~m}$,

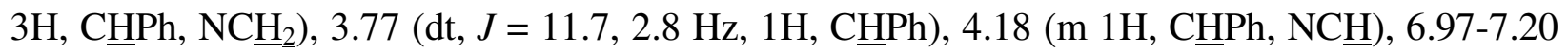
$(\mathrm{m}, 10 \mathrm{H}, \mathrm{PhH}), 9.56(\mathrm{~d}, J=2.9 \mathrm{~Hz}, 1 \mathrm{H}, \mathrm{CHO}) ;{ }^{13} \mathrm{C} \mathrm{NMR}\left(\mathrm{CDCl}_{3}, 125.6 \mathrm{MHz}\right) \delta 28.2\left(\mathrm{CH}_{3}\right)$, $33.4\left(\mathrm{CH}_{2}\right), 46.5(\mathrm{CH}), 47.8(\mathrm{CH}), 51.8\left(\mathrm{CH}_{2}\right), 64.4(\mathrm{CH}), 80.8(\mathrm{C}), 126.4(\mathrm{CH}), 126.5(\mathrm{CH})$, $126.7(\mathrm{CH}), 127.3(\mathrm{CH}), 127.4(\mathrm{CH}), 127.8(\mathrm{CH}), 128.2(\mathrm{CH}), 128.3(\mathrm{CH}), 128.3(\mathrm{CH}), 140.5$ (C), $142.4(\mathrm{C}), 195.0(\mathrm{CH})$. Anal. Calcd for $\mathrm{C}_{23} \mathrm{H}_{27} \mathrm{NO}_{3}$ : C, 75.59; H, 7.45; N, 3.83. Found $\mathrm{C}$, $75.51 ; \mathrm{H}, 7.50 ; \mathrm{N}, 3.78$. 
4,5-Diphenyl-2-trimethylsilanyl-piperidine-1-carboxylic Acid tert-Butyl Ester (86).

The general lithiation procedure was followed using 59 (0.100 g, $0.296 \mathrm{mmol})$ and TMSCl $(0.120 \mathrm{~mL}, 0.946 \mathrm{mmol})$ as the electrophile. Purification by column chromatography (25:1 pet ether/EtOAc) gave $86(0.083 \mathrm{~g}, 69 \%,>97: 3 \mathrm{dr})$ as a white solid. Crystals suitable for X-ray crystallographic analysis were obtained by vapor diffusion with $\mathrm{Et}_{2} \mathrm{O}$ /pentane: $\mathrm{mp} 151-153{ }^{\circ} \mathrm{C}$. Major diastereomer: ${ }^{1} \mathbf{H}$ NMR $\left(\mathrm{CDCl}_{3}, 500 \mathrm{MHz}\right) \delta 0.15\left(\mathrm{~s}, 9 \mathrm{H},\left(\mathrm{C}_{\underline{3}}\right)_{3} \mathrm{Si}\right), 1.53(\mathrm{~s}, 9 \mathrm{H}, \mathrm{O} t-$ $\underline{\mathrm{Bu}}), 1.79\left(\mathrm{q}, J=12.5 \mathrm{~Hz}, 1 \mathrm{H}, \mathrm{CHCH}_{2} \mathrm{CH}\right), 1.95\left(\mathrm{ddd}, J=12.6,3.5,2.4 \mathrm{~Hz}, 1 \mathrm{H}, \mathrm{CHC}_{2} \mathrm{CH}\right)$, $2.58(\mathrm{~d}, J=12.4 \mathrm{~Hz}, 1 \mathrm{H}, \mathrm{NC} \underline{\mathrm{H}}), 2.93(\mathrm{td}, J=11.0,4.1 \mathrm{~Hz}, 1 \mathrm{H}, \mathrm{C} \underline{\mathrm{HPh}}), 3.04$ (m 2H, C $\mathrm{HPh}$, $\left.\mathrm{NC}_{2}{ }_{2}\right), 4.22\left(\mathrm{dd}, J=13.3,3.9 \mathrm{~Hz}, 1 \mathrm{H}, \mathrm{NC}_{2}\right), 7.05-7.22(\mathrm{~m}, 10 \mathrm{H}, \mathrm{Ph} \underline{\mathrm{H}}) ;{ }^{13} \mathbf{C} \mathbf{N M R}\left(\mathrm{CDCl}_{3}\right.$, $125.6 \mathrm{MHz}) \delta-0.7\left(\mathrm{CH}_{3}\right), 28.5\left(\mathrm{CH}_{3}\right), 35.3\left(\mathrm{CH}_{2}\right), 49.3(\mathrm{CH}), 50.7(\mathrm{CH}), 51.3(\mathrm{CH}), 54.6\left(\mathrm{CH}_{2}\right)$, $79.3(\mathrm{C}), 126.1(\mathrm{CH}), 126.3(\mathrm{CH}), 127.5(\mathrm{CH}), 127.7(\mathrm{CH}), 128.1(\mathrm{CH}), 128.2(\mathrm{CH}), 141.7(\mathrm{C})$, 144.0 (C), 155.1 (C). HRMS-FAB (M+1) Calcd for $\mathrm{C}_{25} \mathrm{H}_{36} \mathrm{NO}_{2} \mathrm{Si}$ : 410.2515; Found: 410.2513. $[\alpha]_{\mathrm{D}}^{20}:-7.2^{\circ}\left(c=1.1, \mathrm{CHCl}_{3}\right)$.

\section{4,5-Diphenyl-piperidine-1,2-dicarboxylic Acid 1-tert-Butyl Ester 2-Methyl Ester}

(88). The general lithiation procedure was followed using $59(0.210 \mathrm{~g}, 0.622 \mathrm{mmol})$ and excess $\mathrm{CO}_{2}$ gas as the electrophile. The reaction mixture was acidified with $10 \% \mathrm{HCl}$ during work up. The crude acid was esterified with an ethereal solution of $\mathrm{CH}_{2} \mathrm{~N}_{2}$ (10 eq.). Purification by column chromatography (4:1 pet ether/EtOAc) gave $88(0.186 \mathrm{~g}, 76 \%,>97: 3 \mathrm{dr})$ as a white solid: mp 94-96 ${ }^{\circ} \mathrm{C}$. Major diastereomer: ${ }^{1} \mathbf{H} \mathbf{N M R}\left(\mathrm{CDCl}_{3}, 400 \mathrm{MHz}\right) \delta 1.47(\mathrm{~s}, 9 \mathrm{H}, \mathrm{Ot}$ - $\underline{\mathrm{Bu}})$, $2.11\left(\mathrm{q}, J=12.5 \mathrm{~Hz}, 1 \mathrm{H}, \mathrm{CHC}_{2} \underline{\mathrm{CH}}\right), 2.31\left(\mathrm{ddd}, J=13.6,5.3,3.6 \mathrm{~Hz}, 1 \mathrm{H}, \mathrm{CHC}_{2} \mathrm{CH}\right), 2.93$ (td, $J=12.3,3.4 \mathrm{~Hz}, 1 \mathrm{H}, \mathrm{CHPh}), 3.08(\mathrm{dt}, J=11.9,6.3 \mathrm{~Hz}, 1 \mathrm{H}, \mathrm{C} \underline{\mathrm{HPh}}), 3.64(\mathrm{dd}, J=13.0,6.4 \mathrm{~Hz}$, $\left.1 \mathrm{H}, \mathrm{NC}_{2}\right), 3.75\left(\mathrm{~s}, 3 \mathrm{H}, \mathrm{OCH}_{3}\right), 4.02\left(\mathrm{dd}, J=13.9,5.7 \mathrm{~Hz}, 1 \mathrm{H}, \mathrm{NC}_{2}\right), 4.30$ (dd, $J=11.5,5.3$ $\mathrm{Hz}, 1 \mathrm{H}, \mathrm{NC} \underline{\mathrm{H}}), 6.98-7.24(\mathrm{~m}, 10 \mathrm{H}, \mathrm{Ph} \underline{\mathrm{H}}) ;{ }^{13} \mathbf{C} \mathbf{N M R}\left(\mathrm{CDCl}_{3}, 100.6 \mathrm{MHz}\right) \delta 28.2\left(\mathrm{CH}_{3}\right), 34.1$ $\left(\mathrm{CH}_{2}\right), 46.3(\mathrm{CH}), 47.8(\mathrm{CH}), 52.0(\mathrm{CH}), 47.3\left(\mathrm{CH}_{3}\right), 80.8(\mathrm{C}), 126.5(\mathrm{CH}), 126.5(\mathrm{CH}), 127.3$ $(\mathrm{CH}), 127.5(\mathrm{CH}), 128.2(\mathrm{CH}), 128.3(\mathrm{CH}), 142.7(\mathrm{C}), 143.0(\mathrm{C}), 155.6(\mathrm{C}), 172.5(\mathrm{C})$. Anal. Calcd for $\mathrm{C}_{24} \mathrm{H}_{29} \mathrm{NO}_{4}$ : C, 72.89; H, 7.39; N, 3.54. Found C, 72.61; H, 7.35; N, 3.73. $[\alpha]_{D}^{20}:-8.5^{\circ}$ $\left(c=0.20, \mathrm{CHCl}_{3}\right)$. 
2,6-Dimethyl-3,4-diphenyl-piperidine-1-carboxylic Acid tert-Butyl Ester (89). The general lithiation procedure was followed using 59 (0.050 g, $0.147 \mathrm{mmol})$ and MeOTf (0.051 $\mathrm{mL}, 0.454 \mathrm{mmol}$ ) as the electrophile. Purification by column chromatography (25:1 pet ether/EtOAc) gave $89(0.021 \mathrm{~g}, 41 \%, 93: 7 \mathrm{dr})$ as an oil. Major diastereomer: ${ }^{1} \mathbf{H}$ NMR $\left(\mathrm{CDCl}_{3}, 500 \mathrm{MHz}\right) \delta 1.05\left(\mathrm{~d}, J=7.0 \mathrm{~Hz}, 3 \mathrm{H}, \mathrm{C}_{\underline{3}} \mathrm{CH}\right), 1.46\left(\mathrm{~d}, J=6.7 \mathrm{~Hz}, 3 \mathrm{H}, \mathrm{CH}_{3} \mathrm{CH}\right), 1.51$ (s, 9H, Ot-Bu), $1.71\left(\mathrm{dt}, J=13.9,9.4 \mathrm{~Hz}, 1 \mathrm{H}, \mathrm{CHCH}_{2} \mathrm{CH}\right), 2.10(\mathrm{dt}, J=13.5,4.0 \mathrm{~Hz}, 1 \mathrm{H}$, $\left.\mathrm{CHC}_{2} \underline{\mathrm{H}}_{2} \mathrm{CH}\right), 3.47$ (m, 2H, $\left.\mathrm{CHPh}\right), 2.89(\mathrm{~m}, 2 \mathrm{H}, \mathrm{C} \underline{\mathrm{HPh}}), 3.76$ (dqd, $J=10.1,5.8,3.9 \mathrm{~Hz} 1 \mathrm{H}$, $\left.\mathrm{NCHCH}_{2}\right), 4.50$ (qd, $\left.J=6.8,6.1 \mathrm{~Hz}, 1 \mathrm{H}, \mathrm{NCHCH}\right), 7.06(\mathrm{~m}, 2 \mathrm{H}, \mathrm{PhH}), 7.15(\mathrm{~m}, 8 \mathrm{H}, \mathrm{Ph} \underline{\mathrm{H}}) ;{ }^{13} \mathrm{C}$ NMR $\left(\mathrm{CDCl}_{3}, 125.6 \mathrm{MHz}\right) \delta 13.4\left(\mathrm{CH}_{3}\right), 22.1\left(\mathrm{CH}_{3}\right), 28.5\left(\mathrm{CH}_{3}\right), 39.4(\mathrm{CH}), 41.9\left(\mathrm{CH}_{2}\right), 47.8$ $(\mathrm{CH}), 49.2(\mathrm{CH}), 54.55(\mathrm{CH}), 79.5(\mathrm{C}), 125.9(\mathrm{CH}), 126.1(\mathrm{CH}), 127.5(\mathrm{CH}), 128.0(\mathrm{CH}), 128.3$ $(\mathrm{CH}), 128.9(\mathrm{CH}), 140.7$ (C), $144.5(\mathrm{C}), 155.9$ (C). HRMS-FAB (M+1) Calcd for $\mathrm{C}_{24} \mathrm{H}_{32} \mathrm{NO}_{2}$ : 366.2433; Found: 366.2432.

2-Formyl-4,5-diphenyl-piperidine-1-carboxylic Acid tert-Butyl Ester (92). The general lithiation procedure was followed using $59(0.400 \mathrm{~g}, 1.18 \mathrm{mmol})$ and DMF $(0.294 \mathrm{~mL}$, $3.79 \mathrm{mmol})$ as the electrophile. Purification by column chromatography (5:1 pet ether/EtOAc with $\left.2.5 \% \mathrm{Et}_{3} \mathrm{~N}\right)$ gave $92(0.321 \mathrm{~g}, 74 \%, 91: 9 \mathrm{dr})$ as an oil. Major diastereomer: ${ }^{1} \mathbf{H}$ NMR $\left(\mathrm{CDCl}_{3}, 500 \mathrm{MHz}\right) \delta 1.52(\mathrm{~s}, 9 \mathrm{H}, \mathrm{Ot}-\underline{\mathrm{Bu}}), 2.03\left(\mathrm{td}, J=13.3,7.1 \mathrm{~Hz}, 1 \mathrm{H}, \mathrm{CHCH}_{2} \mathrm{CH}\right), 2.54(\mathrm{~m}$ $1 \mathrm{H}, \mathrm{CHCH}_{2} \mathrm{CH}$ ), $2.81(\mathrm{td}, J=12.2,3.2 \mathrm{~Hz}, 1 \mathrm{H}, \mathrm{CHPh}$ ), $2.96(\mathrm{~m} \mathrm{1H}, \mathrm{C} \underline{\mathrm{HPh}}), 3.11$ (t, $J=12.1$ $\mathrm{Hz}, 1 \mathrm{H}, \mathrm{NC}_{2}$ ), 4.30 (dd rotational isomers, $J=13.5,3,6 \mathrm{~Hz}, 1 \mathrm{H}, \mathrm{NC}_{2}$ ), 4.94 (d rotational isomers, $J=6.2 \mathrm{~Hz}, 1 \mathrm{H}, \mathrm{NC}_{2}$ ), $6.98-7.20(\mathrm{~m}, 10 \mathrm{H}, \mathrm{PhH}$ ), 9.78 (s rotational isomers, $1 \mathrm{H}$, CHO); ${ }^{13} \mathbf{C}$ NMR (rotational isomers, $\left.\mathrm{CDCl}_{3}, 125.6 \mathrm{MHz}\right) \delta 28.3\left(\mathrm{CH}_{3}\right), 31.3\left(\mathrm{CH}_{2}\right), 32.0\left(\mathrm{CH}_{2}\right)$, $33.4\left(\mathrm{CH}_{2}\right), 44.3(\mathrm{CH}), 44.6(\mathrm{CH}), 46.5(\mathrm{CH}), 46.5(\mathrm{CH}), 47.5\left(\mathrm{CH}_{2}\right), 48.0\left(\mathrm{CH}_{2}\right), 49.0\left(\mathrm{CH}_{2}\right)$, $60.2(\mathrm{CH}), 61.4(\mathrm{CH}), 80.8(\mathrm{C}), 126.4(\mathrm{CH}), 126.6(\mathrm{CH}), 127.4(\mathrm{CH}), 127.8(\mathrm{CH}), 128.1(\mathrm{CH})$, $128.2(\mathrm{CH}), 128.3(\mathrm{CH}), 140.4(\mathrm{C}), 140.6(\mathrm{C}), 142.6(\mathrm{C}), 155.4(\mathrm{C}), 201.5(\mathrm{CH})$.

\section{Conversion of 92 to 93.}

Part 1: 4,5-Diphenyl-2-vinyl-piperidine-1-carboxylic Acid tert-Butyl Ester. To a solution of methyl triphenylphosphonium bromide $(0.340 \mathrm{~g}, 0.952 \mathrm{mmol})$ in THF $(3 \mathrm{~mL})$ was at $-78{ }^{\circ} \mathrm{C}$ was added $n$-BuLi $(0.581 \mathrm{~mL}$ of a $1.5 \mathrm{M}$ solution in hexanes, $0.872 \mathrm{mmol})$. The solution was warmed to rt for $1 \mathrm{~h}$ and cooled back to $-78{ }^{\circ} \mathrm{C}$. A solution of $92(0.290 \mathrm{~g}, 0.793 \mathrm{mmol})$ in 
THF (3 mL) was added via cannula and the reaction was stirred for $1 \mathrm{~h}$ at $-78{ }^{\circ} \mathrm{C}$, warmed to $\mathrm{rt}$ and quenched with $\mathrm{H}_{2} \mathrm{O}(5 \mathrm{~mL})$. The aqueous was extracted with ether $(3 \times 15 \mathrm{~mL})$ and the combined organics were dried over $\mathrm{MgSO}_{4}$ and concentrated. The residue was filtered through a plug of silica gel $\left(\mathrm{Et}_{2} \mathrm{O}\right)$ and concentrated. Purification by column chromatography (19:1 pet ether/EtOAc) gave the title compound $(0.199 \mathrm{~g}, 69 \%,>99: 1 \mathrm{dr})$ as a white solid: $\mathrm{mp} 98-100{ }^{\circ} \mathrm{C}$ Major diastereomer: ${ }^{1} \mathrm{H}$ NMR $\left(\mathrm{CDCl}_{3}, 400 \mathrm{MHz}\right) \delta 1.51(\mathrm{~s}, 9 \mathrm{H}, \mathrm{O} t$ - $\underline{\mathrm{Bu}}), 2.07(\mathrm{~m}, 2 \mathrm{H}$, $\mathrm{CHCH}_{2} \mathrm{CH}$ ), 2.86-3.22 (m 3H, C파, $\mathrm{NCH}_{2}$ ), 4.18 (dd rotational isomers, $J=13.4,3.9 \mathrm{~Hz}, 1 \mathrm{H}$, $\mathrm{NC}_{2}$ ), 5.04 (br m rotational isomers, $\left.1 \mathrm{H}, \mathrm{CHN}\right), 5.2(\mathrm{~d}, J=17.6 \mathrm{~Hz}, 1 \mathrm{H}, \mathrm{CH}=\mathrm{C} \underline{\mathrm{H}}), 5.36(\mathrm{t}, J=$ $9.8 \mathrm{~Hz}, 1 \mathrm{H}, \mathrm{CH}=\mathrm{C} \underline{\mathrm{H}}), 5.93(\mathrm{ddd}, J=17.1,10.5,3.1 \mathrm{~Hz}, 1 \mathrm{H}, \mathrm{CH}=\mathrm{C} \underline{\mathrm{H}}), 7.00-7.20(\mathrm{~m}, 10 \mathrm{H}$, $\mathrm{Ph} \underline{\mathrm{H}}) ;{ }^{13} \mathrm{C}$ NMR (rotational isomers, $\left.\mathrm{CDCl}_{3}, 100.6 \mathrm{MHz}\right) \delta 28.4\left(\mathrm{CH}_{3}\right), 37.1\left(\mathrm{CH}_{2}\right), 37.8\left(\mathrm{CH}_{2}\right)$, $42.9(\mathrm{CH}), 43.3(\mathrm{CH}), 45.7\left(\mathrm{CH}_{2}\right), 46.7\left(\mathrm{CH}_{2}\right), 48.4(\mathrm{CH}), 49.0(\mathrm{CH}), 51.9(\mathrm{CH}), 53.1(\mathrm{CH})$, $79.7(\mathrm{C}), 79.8(\mathrm{C}), 116.0\left(\mathrm{CH}_{2}\right), 116.1\left(\mathrm{CH}_{2}\right), 126.1(\mathrm{CH}), 126.4(\mathrm{CH}), 126.4(\mathrm{CH}), 127.5(\mathrm{CH})$, $127.9(\mathrm{CH}), 128.1(\mathrm{CH}), 128.2(\mathrm{CH}), 136.6(\mathrm{CH}), 136.7(\mathrm{CH}), 141.2(\mathrm{C}), 141.5(\mathrm{C}), 143.6(\mathrm{C})$, 143.6 (C), 155.2 (C), 155.2 (C). Anal. Calcd for $\mathrm{C}_{24} \mathrm{H}_{29} \mathrm{NO}_{2}$ : C, 79.30; H, 8.04; N, 3.85. Found C, 79.34; H, 8.15; N, 4.04. $[\alpha]_{\mathrm{D}}^{20}:-33.3^{\circ}\left(c=1.1, \mathrm{CHCl}_{3}\right)$.

Part 2: 2-Ethyl-4,5-diphenyl-piperidine-1-carboxylic Acid tert-Butyl Ester (93). A solution of 4,5-diphenyl-2-vinyl-piperidine-1-carboxylic acid tert-butyl ester (0.135 g, 0.371 $\mathrm{mmol})$ in EtOAc $(8 \mathrm{~mL})$ was hydrogenated at $50 \mathrm{psi}$ for $17 \mathrm{~h}$ with $\mathrm{Pd} / \mathrm{C}(0.040 \mathrm{~g})$. Filtration through a silica plug (EtOAc) and concentration provided 93 (0.128 g, $94 \%)$ as a white solid: mp 99-102 ${ }^{\circ} \mathrm{C}$ Major diastereomer: ${ }^{\mathbf{1}} \mathbf{H}$ NMR (rotational isomers, $\left.\mathrm{CDCl}_{3}, 500 \mathrm{MHz}\right) \delta 0.98(\mathrm{t}$, $\left.J=7.5 \mathrm{~Hz}, 3 \mathrm{H}, \mathrm{CH}_{2} \mathrm{CH}_{3}\right), 1.51(\mathrm{~s}, 9 \mathrm{H}, \mathrm{O} t \underline{\mathrm{Bu}}), 1.70\left(\mathrm{~m}, 1 \mathrm{H}, \mathrm{CHCH}_{2} \mathrm{CH}\right), 1.84-2.04(\mathrm{~m} 3 \mathrm{H}$, $\left.\mathrm{CH}_{2} \mathrm{CH}_{3}, \mathrm{CHC}_{2} \underline{\mathrm{CH}}\right), 2.83-3.20$ (m, 3H, $\underline{\mathrm{HPh}}, \mathrm{NC}_{2}$ ), 4.10 (dd, J=13.3, $4.3 \mathrm{~Hz}, 1 \mathrm{H}, \mathrm{CHN}$ ), $4.29(\mathrm{~m}, 1 \mathrm{H}, \mathrm{CHN}), 7.01-7.20(\mathrm{~m}, 10 \mathrm{H}, \mathrm{PhH}) ;{ }^{13} \mathbf{C} \mathbf{~ N M R}$ (rotational isomers, $\mathrm{CDCl}_{3}, 100.6$ MHz) $\delta 10.7\left(\mathrm{CH}_{3}\right), 10.9\left(\mathrm{CH}_{3}\right), 22.8\left(\mathrm{CH}_{2}\right), 23.2\left(\mathrm{CH}_{2}\right), 28.4\left(\mathrm{CH}_{3}\right), 36.4\left(\mathrm{CH}_{2}\right), 37.5\left(\mathrm{CH}_{2}\right)$, $42.6(\mathrm{CH}), 42.9(\mathrm{CH}), 44.4\left(\mathrm{CH}_{2}\right), 45.7\left(\mathrm{CH}_{2}\right), 48.5(\mathrm{CH}), 49.0(\mathrm{CH}), 51.4(\mathrm{CH}), 52.6(\mathrm{CH}), 79.4$ (C), $126.0(\mathrm{CH}), 126.3(\mathrm{CH}), 126.4(\mathrm{CH}), 127.5(\mathrm{CH}), 127.8(\mathrm{CH}), 127.8(\mathrm{CH}), 128.0(\mathrm{CH})$, $128.1(\mathrm{CH}), 128.2(\mathrm{CH}), 141.3(\mathrm{C}), 141.6(\mathrm{C}), 143.6(\mathrm{C}), 143.7(\mathrm{C}), 154.9$ (C), $155.1(\mathrm{C})$. HRMS-FAB (M+1) Calcd for $\mathrm{C}_{24} \mathrm{H}_{32} \mathrm{NO}_{2}$ : 366.2433; Found: 366.2432. 
6-Ethyl-2-methyl-3,4-diphenyl-piperidine-1-carboxylic Acid tert-Butyl Ester (94). The general lithiation procedure was followed using 93 (0.093 g, $0.255 \mathrm{mmol})$ and MeOTf (0.092 $\mathrm{mL}, 0.815 \mathrm{mmol})$ as the electrophile. Purification by column chromatography (20:1 pet ether/EtOAc) gave $94(0.055 \mathrm{~g}, 57 \%,>99: 1 \mathrm{dr})$ as an oil. Major diastereomer: ${ }^{1} \mathbf{H}$ NMR $\left(\mathrm{CDCl}_{3}, 500 \mathrm{MHz}\right) \delta 0.99\left(\mathrm{t}, J=7.3 \mathrm{~Hz}, 3 \mathrm{H}, \mathrm{CH}_{2} \mathrm{CH}_{3}\right), 1.21\left(\mathrm{~d}, J=6.9 \mathrm{~Hz}, 3 \mathrm{H}, \mathrm{CHCH}_{3}\right), 1.54$ (s, 9H, Ot- $\underline{\mathrm{Bu}}$ ), 1.73 (dquint, $\left.J=14.5,7.3 \mathrm{~Hz}, 1 \mathrm{H}, \mathrm{CHCH}_{2} \mathrm{CH}_{3}\right), 1.88(\mathrm{dt}, J=13.7,4.7 \mathrm{~Hz}, 1 \mathrm{H}$, $\mathrm{CHCH}_{2} \mathrm{CH}$ ), $2.0\left(\mathrm{td}, J=12.5,5.9 \mathrm{~Hz}, 1 \mathrm{H}, \mathrm{CHC}_{2} \mathrm{CH}\right.$ ), 2.05 (dquint, $J=15.2,7.6 \mathrm{~Hz}, 1 \mathrm{H}$, $\left.\mathrm{CHCH}_{2} \mathrm{CH}_{3}\right), 2.83(\mathrm{t}, J=11.0 \mathrm{~Hz}, 1 \mathrm{H}, \mathrm{CHPh}), 3.14(\mathrm{td}, J=12.0,5.0 \mathrm{~Hz}, 1 \mathrm{H}, \mathrm{CHPh}), 3.78(\mathrm{dq}, J$ $\left.=9.5,6.8 \mathrm{~Hz}, 1 \mathrm{H}, \mathrm{CHCH}_{3}\right), 4.2\left(\mathrm{qd}, J=7.6,3.7 \mathrm{~Hz}, 1 \mathrm{H}, \mathrm{CH}_{2} \mathrm{CHCH}_{2}\right), 6.95-7.15(\mathrm{~m}, 10 \mathrm{H}$, $\mathrm{Ph} \underline{\mathrm{H}}) ;{ }^{13} \mathrm{C}$ NMR $\left(\mathrm{CDCl}_{3}, 100.6 \mathrm{MHz}\right) \delta 11.1\left(\mathrm{CH}_{3}\right), 19.7\left(\mathrm{CH}_{3}\right), 23.8\left(\mathrm{CH}_{2}\right), 28.6\left(\mathrm{CH}_{3}\right), 36.4$ $\left(\mathrm{CH}_{2}\right), 45.4(\mathrm{CH}), 53.0(\mathrm{CH}), 54.3(\mathrm{CH}), 55.3(\mathrm{CH}), 79.4(\mathrm{C}), 125.9(\mathrm{CH}), 126.1(\mathrm{CH}), 127.6$ (CH), $128.0(\mathrm{CH}), 128.1(\mathrm{CH}), 142.4(\mathrm{C}), 144.1(\mathrm{C}), 156.6(\mathrm{C})$. HRMS-FAB (M+1) Calcd for $\mathrm{C}_{25} \mathrm{H}_{34} \mathrm{NO}_{2}$ : 380.2590; Found: 380.2591. $[\alpha]_{\mathrm{D}}^{20}:-54.0{ }^{\circ}\left(c=0.80, \mathrm{CHCl}_{3}\right)$.

\section{Conversion of 72 to 95.}

Part 1: 1-Benzyl-4,5-diphenyl-3-phenylselanyl-piperidin-2-one. The general procedure for $t$-BuLi enolization was followed using $72(0.900 \mathrm{~g}, 2.64 \mathrm{mmol})$ and phenylselenenyl chloride $(0.607 \mathrm{~mL}, 3.17 \mathrm{mmol})$. Purfication by column chromatography (7:3 pet ether/EtOAc) gave the title compound $(0.972 \mathrm{~g}, 74 \%, 88: 12 \mathrm{dr})$ as a colorless oil. Major diastereomer: ${ }^{1} \mathbf{H}$ NMR $\left(\mathrm{CDCl}_{3}, 500 \mathrm{MHz}\right) \delta 3.12(\mathrm{td}, J=11.6,4.3 \mathrm{~Hz}, 1 \mathrm{H}, \mathrm{PhC} \underline{\mathrm{H}}), 3.20(\mathrm{dd}, J$ $=12.7,4.3 \mathrm{~Hz}, 1 \mathrm{H}, \mathrm{PhC} \underline{\mathrm{H}}), 3.28\left(\mathrm{t}, J=11.5 \mathrm{~Hz}, 1 \mathrm{H}, \mathrm{NC}_{2}\right), 3.39(\mathrm{dd}, J=11.6,8.2 \mathrm{~Hz}, 1 \mathrm{H}$, $\left.\mathrm{NC}_{2}{ }_{2}\right), 3.32\left(\mathrm{dd}, J=11.9,4.6 \mathrm{~Hz}, 1 \mathrm{H}, \mathrm{NC}_{2}\right), 4.07(\mathrm{~d}, J=8.1 \mathrm{~Hz}, 1 \mathrm{H}, \mathrm{C} \underline{H S e P h}), 4.61(\mathrm{~d}, J=$

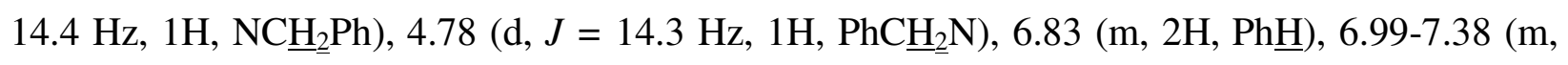
16H, Ph프), $7.61(\mathrm{~m}, 2 \mathrm{H}, \mathrm{Ph} \underline{\mathrm{H}}) ;{ }^{13} \mathbf{C} \mathbf{N M R}\left(\mathrm{CDCl}_{3}, 125.6 \mathrm{MHz}\right) \delta 48.2(\mathrm{CH}), 49.3(\mathrm{CH}), 51.2$ $\left(\mathrm{CH}_{2}\right), 52.3\left(\mathrm{CH}_{2}\right), 53.3(\mathrm{CH}), 126.7(\mathrm{CH}), 127.0(\mathrm{CH}), 127.5(\mathrm{CH}), 127.8(\mathrm{CH}), 128.0(\mathrm{C})$, $128.3(\mathrm{CH}), 128.4(\mathrm{CH}), 128.6(\mathrm{CH}), 128.6(\mathrm{CH}), 128.9(\mathrm{CH}), 136.2(\mathrm{CH}), 136.5(\mathrm{C}), 139.4(\mathrm{C})$, 141.9 (C), 169.4 (C). HRMS-FAB (M+1) Calcd for $\mathrm{C}_{30} \mathrm{H}_{28} \mathrm{NOSe}$ : 498.1336; Found: 498.1335.

Part 2: 1-Benzyl-4,5-diphenyl-5,6-dihydro-1H-pyridin-2-one (95). To a solution of 1benzyl-4,5-diphenyl-3-phenylselanyl-piperidin-2-one $(0.768 \mathrm{~g}, 1.55 \mathrm{mmol})$ in EtOAc $(30 \mathrm{~mL})$ 
was added $30 \% \mathrm{H}_{2} \mathrm{O}_{2}(3.57 \mathrm{~mL})$ and the mixture was stirred vigourously for $30 \mathrm{~min}$. The organics were separated, washed with sat. $\mathrm{NaHCO}_{3}(2 \times 30 \mathrm{~mL})$, dried over $\mathrm{Na}_{2} \mathrm{SO}_{4}$ and concentrated. Purfication by column chromatography (1:1 pet ether/EtOAc) gave 95 (0.474 g, 90 $\%)$ as a white solid: $\mathrm{mp} 103-104{ }^{\circ} \mathrm{C}^{\mathbf{1}} \mathbf{H} \mathbf{~ N M R}\left(\mathrm{CDCl}_{3}, 500 \mathrm{MHz}\right) \delta 3.34(\mathrm{dd}, J=12.4,1.9 \mathrm{~Hz}$, $1 \mathrm{H}, \mathrm{PhC} \underline{\mathrm{H}}), 3.96\left(\mathrm{dd}, J=12.4,5.1 \mathrm{~Hz}, 1 \mathrm{H}, \mathrm{NC}_{2}\right), 4.06\left(\mathrm{dd}, J=4.9,1.7 \mathrm{~Hz}, 1 \mathrm{H}, \mathrm{NC}_{2}\right), 4.46$ $\left(\mathrm{d}, J=15.0 \mathrm{~Hz}, 1 \mathrm{H}, \mathrm{NC}_{2} \underline{\mathrm{Ph}}\right), 4.67\left(\mathrm{~d}, J=15.0 \mathrm{~Hz}, 1 \mathrm{H}, \mathrm{PhC}_{2} \underline{\mathrm{N}}^{\mathrm{N}}\right), 6.74(\mathrm{~s}, 1 \mathrm{H}, \mathrm{C} \underline{\mathrm{H}}=\mathrm{CPh}), 6.96$ $(\mathrm{m}, 2 \mathrm{H}, \mathrm{Ph} \underline{\mathrm{H}}), 7.12-7.34(\mathrm{~m}, 11 \mathrm{H}, \mathrm{PhH}), 7.46(\mathrm{~m}, 2 \mathrm{H}, \mathrm{PhH}) ;{ }^{13} \mathbf{C} \mathbf{N M R}\left(\mathrm{CDCl}_{3}, 125.6 \mathrm{MHz}\right)$ $\delta 42.3(\mathrm{CH}), 49.3\left(\mathrm{CH}_{2}\right), 52.6\left(\mathrm{CH}_{2}\right), 121.3(\mathrm{CH}), 126.2(\mathrm{CH}), 127.0(\mathrm{CH}), 127.3(\mathrm{CH}), 127.6$ $(\mathrm{CH}), 127.8(\mathrm{CH}), 128.3(\mathrm{C}), 128.7(\mathrm{CH}), 128.8(\mathrm{CH}), 129.4(\mathrm{CH}), 136.3(\mathrm{C}), 136.6(\mathrm{C}), 138.9$ (C), 149.8 (C), 165.0 (C). HRMS-FAB (M+1) Calcd for $\mathrm{C}_{24} \mathrm{H}_{22} \mathrm{NO}$ : 340.1701; Found: 340.1701 .

1-Benzyl-4,5-diphenyl-piperidin-2-one (96). A solution of $95(0.410 \mathrm{~g}, 1.21 \mathrm{mmol})$ in EtOAc $(25 \mathrm{~mL})$ was hydrogenated at $250 \mathrm{psi}$ for $17 \mathrm{~h}$ with $\mathrm{Pd}(\mathrm{OH})_{2}(0.410 \mathrm{~g})$. Filtration of the catalyst and concentration provided the crude product which was purified by column chromatography (1:1 pet ether/EtOAc) to provide $96(0.358 \mathrm{~g}, 87 \%,>95: 5 \mathrm{dr})$ as a white solid: mp 105-107. Major diastereomer: ${ }^{1} \mathbf{H} \mathbf{N M R}\left(\mathrm{CDCl}_{3}, 500 \mathrm{MHz}\right) \delta 2.83(\mathrm{dd}, J=17.8,5.8 \mathrm{~Hz}$, $\left.1 \mathrm{H}, \mathrm{COCH}_{2}\right), 2.96\left(\mathrm{dd}, J=17.8,5.8 \mathrm{~Hz}, 1 \mathrm{H}, \mathrm{COC}_{2}\right), 3.45-3.52\left(\mathrm{~m}, 4 \mathrm{H}, \mathrm{PhC} \underline{\mathrm{H}}, \mathrm{NC}_{2}\right), 4.72(\mathrm{~d}$, $\left.J=14.4 \mathrm{~Hz}, 1 \mathrm{H}, \mathrm{NCH}_{2} \mathrm{Ph}\right) 4.81\left(\mathrm{~d}, J=14.1 \mathrm{~Hz}, 1 \mathrm{H}, \mathrm{PhCH}_{2} \mathrm{~N}\right), 6.67$ (m, 4H, Ph్ㅡ), $7.15(\mathrm{~m}, 6 \mathrm{H}$, $\mathrm{Ph} \underline{\mathrm{H}}), 7.28-7.42(\mathrm{~m}, 5 \mathrm{H}, \mathrm{Ph} \underline{\mathrm{H}}) ;{ }^{13} \mathrm{C} \mathbf{N M R}\left(\mathrm{CDCl}_{3}, 125.6 \mathrm{MHz}\right) \delta 36.0\left(\mathrm{CH}_{2}\right), 43.4(\mathrm{CH}), 43.8$ $(\mathrm{CH}), 48.6\left(\mathrm{CH}_{2}\right), 50.4\left(\mathrm{CH}_{2}\right), 126.9(\mathrm{CH}), 127.0(\mathrm{CH}), 127.6(\mathrm{CH}), 127.9(\mathrm{CH}), 127.9(\mathrm{CH})$, $128.1(\mathrm{C}), 128.3(\mathrm{CH}), 128.6(\mathrm{CH}), 136.7$ (C), 139.0 (C), $139.4(\mathrm{C}), 169.3(\mathrm{C})$. Anal. Calcd for $\mathrm{C}_{24} \mathrm{H}_{23} \mathrm{NO}$ : C, 84.42; H, 6.79; N, 4.10. Found C, 84.34; H, 6.80; N, 4.27. $[\alpha]_{\mathrm{D}}^{20}:+60.0^{\circ}(c=1.1$, $\left.\mathrm{CHCl}_{3}\right)$.

\section{Conversion of 96 to 97.}

Part 1: 3-Allyl-1-benzyl-4,5-diphenyl-piperidin-2-one. The general procedure for $t$ BuLi enolization was followed using $96(0.090 \mathrm{~g}, 0.263 \mathrm{mmol})$ and allyl bromide $(0.091 \mathrm{~mL}$, $1.05 \mathrm{mmol})$. Purfication by column chromatography (3:1 pet ether/EtOAc) gave the title compound $(0.093 \mathrm{~g}, 93 \%,>95: 5 \mathrm{dr})$ as a colorless oil. Major diastereomer: ${ }^{1} \mathbf{H} \mathbf{N M R}\left(\mathrm{CDCl}_{3}\right.$, $500 \mathrm{MHz}) \delta 2.75\left(\mathrm{~m}, 2 \mathrm{H}, \mathrm{CH}_{2} \mathrm{CH}=\mathrm{CH}_{2}\right), 2.90(\mathrm{dt}, J=8.5,4.5 \mathrm{~Hz}, 1 \mathrm{H}, \mathrm{CHCO}), 3.35(\mathrm{t}, J=3.4$ 
$\left.\mathrm{Hz}, 1 \mathrm{H}, \mathrm{NC}_{2}\right), 3.44\left(\mathrm{~m}, 3 \mathrm{H}, \mathrm{C} \underline{\mathrm{HPh}}, \mathrm{NC}_{2}\right), 4.62\left(\mathrm{~d}, J=14.1 \mathrm{~Hz}, 1 \mathrm{H}, \mathrm{NC}_{2} \mathrm{Ph}\right), 4.93(\mathrm{~d}, J=$ $\left.14.3 \mathrm{~Hz}, 1 \mathrm{H}, \mathrm{NC}_{2} \mathrm{Ph}\right), 5.14\left(\mathrm{~m}, 1 \mathrm{H}, \mathrm{CH}=\underline{\mathrm{CH}}_{2}\right), 5.17\left(\mathrm{~d}, J=1.1 \mathrm{~Hz}, 1 \mathrm{H}, \mathrm{CH}=\underline{\mathrm{C}}_{2}\right), 5.88(\mathrm{~m}, 1 \mathrm{H}$, $\left.\mathrm{C} \underline{H}=\mathrm{CH}_{2}\right), 6.64(\mathrm{~m}, 4 \mathrm{H}, \mathrm{Ph} \underline{H}), 7.14(\mathrm{~m}, 6 \mathrm{H}, \mathrm{Ph} \underline{\mathrm{H}}), 7.35(\mathrm{~m}, 5 \mathrm{H}, \mathrm{Ph} \underline{\mathrm{H}}) ;{ }^{13} \mathbf{C} \mathbf{N M R}\left(\mathrm{CDCl}_{3}, 125.6\right.$ MHz) $\delta 36.7\left(\mathrm{CH}_{2}\right), 40.6(\mathrm{CH}), 44.6(\mathrm{CH}), 46.8(\mathrm{CH}), 48.1\left(\mathrm{CH}_{2}\right), 50.7\left(\mathrm{CH}_{2}\right), 117.6\left(\mathrm{CH}_{2}\right)$, $126.7(\mathrm{CH}), 126.8(\mathrm{CH}), 127.6(\mathrm{CH}), 127.8(\mathrm{CH}), 127.9(\mathrm{C}), 128.1(\mathrm{CH}), 128.5(\mathrm{CH}), 128.6$ $(\mathrm{CH}), 128.8(\mathrm{CH}), 135.9(\mathrm{CH}), 136.8(\mathrm{C}), 139.3(\mathrm{C}), 139.4(\mathrm{C}), 171.8(\mathrm{C})$. HRMS-FAB $(\mathrm{M}+1)$ Calcd for $\mathrm{C}_{27} \mathrm{H}_{28} \mathrm{NO}$ : 382.2171; Found: 382.2171 .

Part 2: 3-Allyl-1-benzyl-4,5-diphenyl-piperidine (97). The general reduction procedure for benzyl lactams was followed using 3-allyl-1-benzyl-4,5-diphenyl-piperidin-2-one $(0.085 \mathrm{~g}, 0.222 \mathrm{mmol})$ and lithium aluminum hydride $(0.666 \mathrm{~mL}$ of a $1 \mathrm{M}$ solution in THF, $0.666 \mathrm{mmol})$. Purification by column chromatography (20:1 pet ether/EtOAc) provided 97 $(0.069 \mathrm{~g}, 85 \%)$ as a colorless oil. Major diastereomer: ${ }^{1} \mathbf{H} \mathbf{N M R}\left(\mathrm{CDCl}_{3}, 500 \mathrm{MHz}\right) \delta 1.69(\mathrm{dt}$, $\left.J=14.4,8.4 \mathrm{~Hz}, 1 \mathrm{H}, \underline{\mathrm{C}}_{2} \mathrm{CH}=\mathrm{CH}_{2}\right), 1.96(\mathrm{t}, J=10.9 \mathrm{~Hz}, 1 \mathrm{H}, \mathrm{CHPh}), 2.04(\mathrm{~m}, 1 \mathrm{H}$, $\mathrm{C} \mathrm{HCH}_{2} \mathrm{CH}=\mathrm{CH}_{2}$ ), $2.41\left(\mathrm{~m}, 1 \mathrm{H}, \underline{\mathrm{CH}}_{2} \mathrm{CH}=\mathrm{CH}_{2}\right), 2.59$ (dd, $\left.J=11.3,3.8 \mathrm{~Hz}, 1 \mathrm{H}, \mathrm{CHPh}\right), 2.81$ (dd, $\left.J=12.0,5.4 \mathrm{~Hz}, 1 \mathrm{H}, \mathrm{C}_{2} \mathrm{~N}\right), 3.03\left(\mathrm{~m}, 1 \mathrm{H}, \mathrm{NC}_{2}\right), 3.28\left(\mathrm{~d}, J=11.5 \mathrm{~Hz}, 1 \mathrm{H}, \mathrm{NC}_{2} \underline{\mathrm{H}}_{2}\right), 3.33(\mathrm{dd}, J$ $\left.=12.0,3.5 \mathrm{~Hz}, 1 \mathrm{H}, \mathrm{NCH}_{2}\right), 3.56\left(\mathrm{~d}, J=13.1 \mathrm{~Hz}, 1 \mathrm{H}, \mathrm{NCH}_{2} \mathrm{Ph}\right), 3.70(\mathrm{~d}, J=13.2 \mathrm{~Hz}, 1 \mathrm{H}$, $\left.\mathrm{NC}_{2} \underline{\mathrm{Ph}}\right), 4.89$ (d, $\left.J=16.9 \mathrm{~Hz}, 1 \mathrm{H}, \mathrm{CH}=\underline{\mathrm{CH}}_{2}\right), 4.95\left(\mathrm{dd}, J=10.0,1.0 \mathrm{~Hz}, 1 \mathrm{H}, \mathrm{CH}=\mathrm{C}_{\underline{2}}\right), 5.71$ (m, $\left.1 \mathrm{H}, \mathrm{C} \underline{\mathrm{H}}=\mathrm{CH}_{2}\right), 7.14(\mathrm{~m}, 6 \mathrm{H}, \mathrm{Ph} \underline{\mathrm{H}}), 7.29(\mathrm{~m}, 2 \mathrm{H}, \mathrm{Ph} \underline{\mathrm{H}}), 7.39$ (m, 4H, $\left.\mathrm{Ph} \underline{\mathrm{H}}\right), 7.47(\mathrm{~m}, 3 \mathrm{H}$, $\mathrm{Ph} \underline{\mathrm{H}}) ;{ }^{13} \mathrm{C}$ NMR $\left(\mathrm{CDCl}_{3}, 125.6 \mathrm{MHz}\right) \delta 33.8(\mathrm{CH}), 36.3\left(\mathrm{CH}_{2}\right), 47.3(\mathrm{CH}), 52.7(\mathrm{CH}), 58.9$ $\left(\mathrm{CH}_{2}\right), 59.6\left(\mathrm{CH}_{2}\right), 63.8\left(\mathrm{CH}_{2}\right), 116.1\left(\mathrm{CH}_{2}\right), 125.8(\mathrm{CH}), 125.9(\mathrm{CH}), 126.9(\mathrm{CH}), 126.9(\mathrm{CH})$, $127.5(\mathrm{C}), 128.2(\mathrm{CH}), 128.9(\mathrm{CH}), 129.1(\mathrm{CH}), 130.8(\mathrm{CH}), 136.3(\mathrm{CH}), 138.6(\mathrm{C}), 141.8(\mathrm{C})$, 142.3 (C). HRMS-FAB $(\mathrm{M}+1)$ Calcd for $\mathrm{C}_{27} \mathrm{H}_{30} \mathrm{~N}: 369.2378$; Found: $368.2377 .[\alpha]_{\mathrm{D}}^{20}:-106.6^{\circ}$ $\left(c=0.50, \mathrm{CHCl}_{3}\right)$.

\section{Conversion of 96 to 98 .}

Part 1: 1-Benzyl-3,4-diphenyl-piperidine. The general reduction procedure for benzyl lactams was followed using $96(0.400 \mathrm{~g}, 1.17 \mathrm{mmol})$ and lithium aluminum hydride $(3.51 \mathrm{~mL}$ of a $1 \mathrm{M}$ solution in THF, $3.51 \mathrm{mmol}$ ) with a $6 \mathrm{~h}$ reflux period. Purification by column chromatography (8:1 pet ether/EtOAc) provided the title compound $(0.332 \mathrm{~g}, 87 \%)$ as a colorless oil. ${ }^{1} \mathbf{H}$ NMR $\left(\mathrm{CDCl}_{3}, 500 \mathrm{MHz}\right) \delta 1.65\left(\mathrm{~m}, 1 \mathrm{H}, \mathrm{CH}_{2} \mathrm{CH}_{2} \mathrm{CH}\right), 2.26(\mathrm{~m}, 2 \mathrm{H}$, 
$\left.\mathrm{CH}_{2} \underline{\mathrm{CH}}_{2} \mathrm{CH}, \mathrm{PhCH}\right), 2.70(\mathrm{dd}, J=11.5,3.8 \mathrm{~Hz}, 1 \mathrm{H}, \underline{\mathrm{CHPh}}), 3.12\left(\mathrm{~m}, 1 \mathrm{H}, \mathrm{CH}_{2} \mathrm{~N}\right), 3.15(\mathrm{~m}, 1 \mathrm{H}$, $\left.\mathrm{CH}_{2} \mathrm{~N}\right), 3.24\left(\mathrm{~m}, 1 \mathrm{H}, \mathrm{CH}_{2} \mathrm{~N}\right), 3.15\left(\mathrm{dt}, J=11.1,1.4 \mathrm{~Hz}, 1 \mathrm{H}, \mathrm{CH}_{2} \mathrm{~N}\right), 3.59(\mathrm{~d}, J=12.9 \mathrm{~Hz}, 1 \mathrm{H}$, $\left.\mathrm{NC}_{2} \underline{\mathrm{Ph}}\right), 3.66\left(\mathrm{~d}, J=13.1 \mathrm{~Hz}, 1 \mathrm{H}, \mathrm{PhC}_{\underline{2}} \mathrm{~N}\right), 6.83(\mathrm{~m}, 2 \mathrm{H}, \mathrm{Ph} \underline{\mathrm{H}}), 7.15-7.50(\mathrm{~m}, 13 \mathrm{H}, \mathrm{Ph} \underline{\mathrm{H}}) ;{ }^{13} \mathrm{C}$ NMR $\left(\mathrm{CDCl}_{3}, 125.6 \mathrm{MHz}\right) \delta 25.8\left(\mathrm{CH}_{2}\right), 46.6(\mathrm{CH}), 46.7(\mathrm{CH}), 54.2\left(\mathrm{CH}_{2}\right), 59.0\left(\mathrm{CH}_{2}\right), 63.7$ $\left(\mathrm{CH}_{2}\right), 125.7(\mathrm{CH}), 125.9(\mathrm{CH}), 126.9(\mathrm{CH}), 126.9(\mathrm{CH}), 127.6(\mathrm{CH}), 127.9(\mathrm{C}), 128.2(\mathrm{CH})$, $129.1(\mathrm{CH}), 130.7(\mathrm{CH}), 138.8(\mathrm{C}), 141.8$ (C), 143.9 (C). HRMS-FAB (M+1) Calcd for $\mathrm{C}_{24} \mathrm{H}_{26} \mathrm{~N}$ : 328.2065; Found: 328.2066.

Part 2: 3,4-Diphenyl-piperidine-1-carboxylic Acid tert-Butyl Ester (98). A solution of 1-benzyl-3,4-diphenyl-piperidine $(0.300 \mathrm{~g}, 0.916 \mathrm{mmol})$ and $\mathrm{Boc}_{2} \mathrm{O}(0.420 \mathrm{~mL}, 1.83 \mathrm{mmol})$ in EtOAc $(21 \mathrm{~mL})$ was hydrogenated at atmospheric pressure for $18 \mathrm{~h}$ with $\mathrm{Pd}(\mathrm{OH})_{2}(0.156 \mathrm{~g})$. Filtration of the catalyst and concentration provided the crude product which was purified by column chromatography (10:1 pet ether/EtOAc) to provide $98(0.241 \mathrm{~g}, 78 \%)$ as a white solid: mp 126-127 ${ }^{\circ} \mathrm{C} .{ }^{1} \mathbf{H} \mathbf{N M R}\left(\mathrm{CDCl}_{3}, 500 \mathrm{MHz}\right) \delta 1.52$ (s, 9H, Ot-Bu$), 1.69\left(\mathrm{~m}, 1 \mathrm{H}, \mathrm{CH}_{2} \underline{\mathrm{C}}_{2} \mathrm{CH}\right)$, 2.19 (qd, $J=12.7,4.3 \mathrm{~Hz}, 1 \mathrm{H}, \mathrm{CH}_{2} \underline{\mathrm{CH}}_{2} \mathrm{CH}$ ), 3.08 (m, 2H, CHPh), 3.21 (dt, $J=9.2,3.5 \mathrm{~Hz}, 1 \mathrm{H}$, $\left.\mathrm{C}_{2} \mathrm{~N}\right), 3.41\left(\mathrm{~d}, J=11.0 \mathrm{~Hz}, 1 \mathrm{H}, \mathrm{C}_{2} \mathrm{~N}\right), 4.50\left(\mathrm{~m}, 2 \mathrm{H}, \mathrm{CH}_{2} \mathrm{~N}\right), 6.84(\mathrm{~m}, 4 \mathrm{H}, \mathrm{Ph} \underline{\mathrm{H}}), 7.04-7.18(\mathrm{~m}$, $6 \mathrm{H}, \mathrm{Ph} \underline{\mathrm{H}}) ;{ }^{13} \mathrm{C} \mathbf{N M R}\left(\mathrm{CDCl}_{3}, 125.6 \mathrm{MHz}\right) \delta 24.9\left(\mathrm{CH}_{2}\right), 28.3\left(\mathrm{CH}_{3}\right), 43.8\left(\mathrm{CH}_{2}\right), 45.3(\mathrm{CH}), 46.2$ $(\mathrm{CH}), 48.8\left(\mathrm{CH}_{2}\right), 79.6(\mathrm{C}), 126.0(\mathrm{CH}), 126.1(\mathrm{CH}), 127.2(\mathrm{CH}), 127.7(\mathrm{CH}), 129.4(\mathrm{CH}), 140.5$ (C), 143.1 (C), 154.9 (C). HRMS-FAB (M+1) Calcd for $\mathrm{C}_{22} \mathrm{H}_{28} \mathrm{NO}_{2}$ : 338.2120; Found: 338.2120. $[\alpha]_{\mathrm{D}}^{20}:-136.5^{\circ}\left(c=1.0, \mathrm{CHCl}_{3}\right)$.

2-Methyl-4,5-diphenyl-piperidine-1-carboxylic Acid tert-Butyl Ester (99). The general lithiation procedure was followed using 98 (0.100 g, $0.296 \mathrm{mmol})$ and MeOTf (0.107 $\mathrm{mL}, 0.947 \mathrm{mmol}$ ) as the electrophile. Purification by column chromatography (20:1 pet ether/EtOAc) gave $99(0.068 \mathrm{~g}, 66 \%, 92: 8 \mathrm{dr})$ as as a white solid. Crystals suitable for X-ray crystallographic analysis were obtained by slow evaporation from pet ether: $\mathrm{mp} 112-114{ }^{\circ} \mathrm{C}$.

Major diastereomer: ${ }^{1} \mathbf{H}$ NMR $\left(\mathrm{CDCl}_{3}, 500 \mathrm{MHz}\right) \delta 1.34\left(\mathrm{~d}, J=6.2 \mathrm{~Hz}, 3 \mathrm{H}, \mathrm{CHCH}_{3}\right), 1.54(\mathrm{~s}$, 9H, Ot- $\underline{\mathrm{Bu}}), 2.03\left(\mathrm{ddd}, J=13.5,7.3,3.0 \mathrm{~Hz}, 1 \mathrm{H}, \mathrm{CH}_{2} \underline{\mathrm{C}}_{2} \mathrm{CH}\right), 2.08(\mathrm{td}, J=13.3,10.7 \mathrm{~Hz}, 1 \mathrm{H}$, $\mathrm{CH}_{2} \underline{\mathrm{CH}}_{2} \mathrm{CH}$ ), 3.27 (ddd, $\left.J=12.4,9.0,2.7 \mathrm{~Hz}, 1 \mathrm{H}, \mathrm{CHPh}\right), 3.34$ (dd, $J=14.1,10.9 \mathrm{~Hz}, 1 \mathrm{H}$, $\mathrm{C}_{2} \mathrm{~N}$ ), 3.61 (dt, $J=10.9,8.3 \mathrm{~Hz}, 1 \mathrm{H}, \mathrm{CHPh}$ ), 4.19 (dquint, $J=13.1,6.6 \mathrm{~Hz}, 1 \mathrm{H}, \mathrm{CH}_{3} \mathrm{C} \underline{\mathrm{HN}}$ ), 
$4.26\left(\mathrm{dd}, J=14.1,7.5 \mathrm{~Hz}, 1 \mathrm{H}, \mathrm{C}_{2} \mathrm{~N}\right), 6.83(\mathrm{~m}, 2 \mathrm{H}, \mathrm{Ph} \underline{\mathrm{H}}), 6.95$ (m, 3H, Ph프), 7.03 (m, 5H, $\mathrm{Ph} \underline{\mathrm{H}}) ;{ }^{13} \mathrm{C}$ NMR $\left(\mathrm{CDCl}_{3}, 125.6 \mathrm{MHz}\right) \delta 19.4\left(\mathrm{CH}_{3}\right), 28.5\left(\mathrm{CH}_{3}\right), 32.4\left(\mathrm{CH}_{2}\right), 42.0(\mathrm{CH}), 42.2$ $\left(\mathrm{CH}_{2}\right), 45.3(\mathrm{CH}), 50.1(\mathrm{CH}), 79.3(\mathrm{C}), 125.5(\mathrm{CH}), 126.3(\mathrm{CH}), 127.5(\mathrm{CH}), 127.7(\mathrm{CH}), 127.9$ $(\mathrm{CH}), 128.8(\mathrm{CH}), 140.0(\mathrm{C}), 142.1(\mathrm{C}), 155.3(\mathrm{C})$. HRMS-FAB (M+1) Calcd for $\mathrm{C}_{23} \mathrm{H}_{30} \mathrm{NO}_{2}$ : 352.2277; Found: 352.2276.

\section{Representative Procedure for Lithiation of Boc-3,4-disubstituted Pyrrolidines: 2,3-} Dimethyl-4-phenyl-pyrrolidine-1-carboxylic Acid tert-Butyl Ester (100) and 2,4-Dimethyl3-phenyl-pyrrolidine-1-carboxylic Acid tert-Butyl Ester (101). To a stirring solution of 67 $(0.103 \mathrm{~g}, 0.394 \mathrm{mmol})$ in ether $(4.0 \mathrm{~mL})$ under $\mathrm{N}_{2}$ was added $1(0.117 \mathrm{~mL}, 0.512 \mathrm{mmol})$. The solution was cooled to $-78{ }^{\circ} \mathrm{C}$ and sec-BuLi $(0.355 \mathrm{~mL}$ of a $1.44 \mathrm{M}$ solution in cyclohexane, $0.512 \mathrm{mmol})$ was added. The solution was stirred for $4 \mathrm{~h}$ at $-78{ }^{\circ} \mathrm{C}$ and MeOTf $(0.147 \mathrm{~mL}, 1.30$ mmol) was added and the solution was stirred for an addition $15 \mathrm{~min}$ at $-78{ }^{\circ} \mathrm{C}$, warmed to rt and quenched with $\mathrm{H}_{2} \mathrm{O}(10 \mathrm{~mL})$. The aqueous was extracted with ether $(3 \times 10 \mathrm{~mL})$ and the combined organics were dried over $\mathrm{MgSO}_{4}$. Purification by column chromatography (12:1 pet ether/EtOAc) gave a 58:42 mixture of $\mathbf{1 0 0}$ and $\mathbf{1 0 1}(0.074 \mathrm{~g}, 69 \%,>95: 5 \mathrm{dr})$ as a colorless oil. Major diastereomer: ${ }^{1} \mathbf{H}$ NMR (DMSO- $\left.d_{6}, 100{ }^{\circ} \mathrm{C}, 500 \mathrm{MHz}\right) \delta 0.83(\mathrm{~d}, J=6.5 \mathrm{~Hz}, 3 \mathrm{H}$, $\left.\mathrm{C}_{\underline{3}} \mathrm{CH}\right), 0.91\left(\mathrm{~d}, J=6.6 \mathrm{~Hz}, 3 \mathrm{H}, \mathrm{C}_{3} \underline{\mathrm{CH}}\right), 1.20\left(\mathrm{~d}, J=6.0 \mathrm{~Hz}, 3 \mathrm{H}, \mathrm{C}_{\underline{3}} \mathrm{CH}\right), 1.33(\mathrm{~d}, J=6.1$ $\left.\mathrm{Hz}, 3 \mathrm{H}, \mathrm{CH}_{3} \underline{\mathrm{CH}}\right), 1.43$ (s, 18H, Ot- $\left.\underline{\mathrm{Bu}}\right), 1.88\left(\mathrm{~m}, 1 \mathrm{H}, \mathrm{CH}_{3} \mathrm{C} \underline{\mathrm{H}}\right), 2.21$ (m, 1H, $\left.\mathrm{CH}_{3} \mathrm{C} \underline{\mathrm{H}}\right), 2.38$ (t, $J$ $=7.0 \mathrm{~Hz}, 1 \mathrm{H}, \mathrm{NC} \underline{\mathrm{H}}), 2.74(\mathrm{dt}, J=11.1,7.9 \mathrm{~Hz}, 1 \mathrm{H}, \mathrm{PhC} \underline{\mathrm{H}}), 2.90(\mathrm{t}, J=10.5 \mathrm{~Hz}, 1 \mathrm{H}, \mathrm{PhC} \underline{\mathrm{H}})$, 3.21 (t, $\left.J=10.8 \mathrm{~Hz}, 1 \mathrm{H}, \mathrm{NC}_{2}\right), 3.31\left(\mathrm{~m}, 1 \mathrm{H}, \mathrm{C}_{3} \underline{\mathrm{CHN}}\right), 3.64\left(\mathrm{~m}, 1 \mathrm{H}, \mathrm{C}_{3} \underline{\mathrm{CHN}}\right), 3.83(\mathrm{~m}, 2 \mathrm{H}$,

$\mathrm{NCH})$, 7.20-7.35 (m, 20H, PhH $) ;{ }^{13} \mathbf{C}$ NMR $\left(\mathrm{CDCl}_{3}, 125.6 \mathrm{MHz}\right) \delta 14.9\left(\mathrm{CH}_{3}\right), 15.0\left(\mathrm{CH}_{3}\right), 18.8$ $(\mathrm{CH}), 19.8(\mathrm{CH}), 28.5\left(\mathrm{CH}_{3}\right), 19.8(\mathrm{CH}), 19.8(\mathrm{CH}), 39.5(\mathrm{CH}), 39.7(\mathrm{CH}), 49.1(\mathrm{CH}), 49.7$ $(\mathrm{CH}), 50.8(\mathrm{CH}), 51.2(\mathrm{CH}), 53.2\left(\mathrm{CH}_{2}\right), 53.4\left(\mathrm{CH}_{2}\right), 53.9\left(\mathrm{CH}_{2}\right), 60.4(\mathrm{CH}), 61.3(\mathrm{CH}), 62.4$ $(\mathrm{CH}), 78.9(\mathrm{C}), 79.1(\mathrm{C}), 126.8(\mathrm{CH}), 126.9(\mathrm{CH}), 127.6(\mathrm{CH}), 128.1(\mathrm{CH}), 128.5(\mathrm{CH}), 139.6$ (C), 139.9 (C), 154.4 (C). HRMS-FAB (M+1) Calcd for $\mathrm{C}_{17} \mathrm{H}_{26} \mathrm{NO}_{2}$ : 276.1964; Found: 276.1964 .

2-Methyl-3,4-diphenyl-pyrrolidine-1-carboxylic Acid tert-Butyl Ester (102). The general lithiation procedure was followed using $66(0.100 \mathrm{~g}, 0.309 \mathrm{mmol})$ and $\mathrm{MeOTf}(0.115 \mathrm{~mL}$, $1.02 \mathrm{mmol})$ as the electrophile. Purification by column chromatography (12:1 pet ether/EtOAc) 
gave $102(0.085 \mathrm{~g}, 82 \%,>95: 5 \mathrm{dr})$ as an oil. Major diastereomer: ${ }^{1} \mathbf{H}$ NMR (DMSO- $d_{6}, 100$ $\left.{ }^{\circ} \mathrm{C}, 500 \mathrm{MHz}\right) \delta 1.30\left(\mathrm{~d}, J=6.0 \mathrm{~Hz}, 3 \mathrm{H}, \mathrm{CH}_{\underline{3}} \mathrm{CH}\right), 1.45(\mathrm{~s}, 9 \mathrm{H}, \mathrm{Ot}-\mathrm{Bu}), 3.09$ (dd, $J=11.8,9.2$ $\mathrm{Hz}, 1 \mathrm{H}, \mathrm{PhCH}), 3.36$ (t, $\left.J=10.9 \mathrm{~Hz}, 1 \mathrm{H}, \mathrm{NC}_{2}\right), 3.52$ (td, $\left.J=11.4,7.3 \mathrm{~Hz}, 1 \mathrm{H}, \mathrm{PhC} \underline{\mathrm{H}}\right), 4.75$ (dq, $J=9.2,5.9 \mathrm{~Hz}, 1 \mathrm{H}, \mathrm{NC}_{\mathrm{HCH}_{3}}$ ), 4.03 (dd, $J=10.6,7.4 \mathrm{~Hz}, 1 \mathrm{H}, \mathrm{NC}_{2}$ ), $7.10-7.27$ (m, 10H, $\mathrm{Ph} \underline{\mathrm{H}}) ;{ }^{13} \mathrm{C}$ NMR (DMSO- $\left.d_{6}, 100{ }^{\circ} \mathrm{C}, 125.6 \mathrm{MHz}\right) \delta 18.2\left(\mathrm{CH}_{3}\right), 27.7\left(\mathrm{CH}_{3}\right), 48.7(\mathrm{CH}), 52.8$ $\left(\mathrm{CH}_{2}\right), 59.3(\mathrm{CH}), 60.3(\mathrm{CH}), 77.9(\mathrm{C}), 126.0(\mathrm{CH}), 126.1(\mathrm{CH}), 126.9(\mathrm{CH}), 127.3(\mathrm{CH}), 127.6$ $(\mathrm{CH}), 127.8(\mathrm{CH}), 138.6(\mathrm{C}), 138.7(\mathrm{C}), 153.0(\mathrm{C})$. HRMS-FAB (M+1) Calcd for $\mathrm{C}_{22} \mathrm{H}_{28} \mathrm{NO}_{2}$ : 338.2120; Found: 338.2120.

\section{3,4-Diphenyl-2-trimethylsilanyl-pyrrolidine-1-carboxylic Acid tert-Butyl Ester (103).}

The general lithiation procedure was followed using $66(0.100 \mathrm{~g}, 0.309 \mathrm{mmol})$ and $\mathrm{TMSCl}(0.129$ $\mathrm{mL}, 1.02 \mathrm{mmol})$ as the electrophile. Purification by column chromatography (15:1 pet ether/EtOAc) gave $\mathbf{1 0 3}(0.095 \mathrm{~g}, 78 \%,>95: 5 \mathrm{dr})$ as an oil. Major diastereomer: ${ }^{1} \mathbf{H}$ NMR (DMSO- $\left.d_{6}, 100{ }^{\circ} \mathrm{C}, 500 \mathrm{MHz}\right) \delta 0.14\left(\mathrm{~s}, 9 \mathrm{H},\left(\mathrm{C}_{3}\right)_{3} \mathrm{Si}\right), 1.47$ (s, 9H, Ot-Bu$), 3.18(\mathrm{td}, J=10.9$, $6.4 \mathrm{~Hz}, 1 \mathrm{H}, \mathrm{PhCH}), 3.25$ (t, $\left.J=10.7 \mathrm{~Hz}, 1 \mathrm{H}, \mathrm{NC}_{2}\right), 3.35$ (t, $\left.J=10.3 \mathrm{~Hz}, 1 \mathrm{H}, \mathrm{PhCH}\right), 4.14$ (dd, $\left.J=8.5,7.3 \mathrm{~Hz}, 1 \mathrm{H}, \mathrm{NC}_{2}\right), 7.08-7.24(\mathrm{~m}, 10 \mathrm{H}, \mathrm{PhH}) ;{ }^{13} \mathbf{C} \mathbf{N M R}\left(\mathrm{CDCl}_{3}\right.$, rotational isomers, 25 $\left.{ }^{\circ} \mathrm{C}, 125.6 \mathrm{MHz}\right) \delta$-1.8 $\left(\mathrm{CH}_{3}\right), 28.5\left(\mathrm{CH}_{3}\right), 54.1(\mathrm{CH}), 54.5\left(\mathrm{CH}_{2}\right), 54.8(\mathrm{CH}), 55.2(\mathrm{CH}), 56.1$ $(\mathrm{CH}), 126.6(\mathrm{CH}), 126.8(\mathrm{CH}), 127.5(\mathrm{CH}), 128.0(\mathrm{CH}), 128.3(\mathrm{CH}), 128.4(\mathrm{CH}), 139.0(\mathrm{C})$, 141.0 (C), 155.1 (C). HRMS-FAB (M+1) Calcd for $\mathrm{C}_{24} \mathrm{H}_{34} \mathrm{NO}_{2} \mathrm{Si}$ : 396.2359; Found: 396.2357.

2-Formyl-3,4-diphenyl-pyrrolidine-1-carboxylic Acid tert-Butyl Ester (104). The general lithiation procedure was followed using $66(0.100 \mathrm{~g}, 0.309 \mathrm{mmol})$ and DMF $(0.079 \mathrm{~mL}$, $1.02 \mathrm{mmol})$ as the electrophile. Purification by column chromatography (4:1 pet ether/EtOAc) gave $104(0.084 \mathrm{~g}, 77 \%,>95: 5 \mathrm{dr})$ as an oil. Major diastereomer: ${ }^{1} \mathbf{H}$ NMR (DMSO- $d_{6}, 100$ $\left.{ }^{\circ} \mathrm{C}, 400 \mathrm{MHz}\right) \delta 1.42(\mathrm{~s}, 9 \mathrm{H}, \mathrm{Ot}-\underline{\mathrm{Bu}}), 3.53(\mathrm{t}, J=10.5 \mathrm{~Hz}, 1 \mathrm{H}, \mathrm{PhC} \underline{\mathrm{H}}), 3.63(\mathrm{t}, J=10.7 \mathrm{~Hz}, 1 \mathrm{H}$, $\left.\mathrm{NC}_{2}\right), 3.75(\mathrm{q}, J=8.3 \mathrm{~Hz}, 1 \mathrm{H}, \mathrm{PhCH}), 4.02\left(\mathrm{~m}, 1 \mathrm{H}, \mathrm{NC}_{2}\right), 4.14(\mathrm{dd}, J=10.7,3.4 \mathrm{~Hz}, 1 \mathrm{H}$, $\mathrm{NC} \underline{\mathrm{H}}$ ), 7.12-7.28 (m, 10H, PhH), $9.49(\mathrm{~d}, J=3.7 \mathrm{~Hz}, 1 \mathrm{H}, \mathrm{CHO}) ;{ }^{13} \mathrm{C}$ NMR (DMSO- $d_{6}$, rotational isomers, $\left.25{ }^{\circ} \mathrm{C}, 100.6 \mathrm{MHz}\right) \delta 28.5\left(\mathrm{CH}_{3}\right), 28.7\left(\mathrm{CH}_{3}\right), 49.9(\mathrm{CH}), 50.9(\mathrm{CH}), 51.8$ $(\mathrm{CH}), 52.7(\mathrm{CH}), 54.1\left(\mathrm{CH}_{2}\right), 72.1(\mathrm{CH}), 72.4(\mathrm{CH}), 80.5(\mathrm{C}), 80.6(\mathrm{C}), 127.7(\mathrm{CH}), 127.9(\mathrm{CH})$, $128.5(\mathrm{CH}), 129.0(\mathrm{CH}), 129.1(\mathrm{CH}), 137.4(\mathrm{C}), 138.6(\mathrm{C}), 138.7(\mathrm{C}), 153.3(\mathrm{C}), 154.5(\mathrm{C})$, 199.6 (CH). HRMS-FAB (M+1) Calcd for $\mathrm{C}_{22} \mathrm{H}_{26} \mathrm{NO}_{3}$ : 352.1913; Found: 352.1914. 


\section{3,4-Diphenyl-pyrrolidine-1,2-dicarboxylic Acid 1-tert-Butyl Ester 2-Methyl Ester}

(106). The general lithiation procedure was followed using $66(0.100 \mathrm{~g}, 0.309 \mathrm{mmol})$ and excess $\mathrm{CO}_{2}$ gas as the electrophile. The reaction mixture was acidified with $10 \% \mathrm{HCl}$ during work up. The crude acid was esterified with an ethereal solution of $\mathrm{CH}_{2} \mathrm{~N}_{2}$ (10 eq.). Purification by column chromatography (4:1 pet ether/EtOAc) gave $106(0.090 \mathrm{~g}, 76 \%,>95: 5 \mathrm{dr})$ as an oil. Major diastereomer: ${ }^{1} \mathbf{H}$ NMR (DMSO- $\left.d_{6}, 100{ }^{\circ} \mathrm{C}, 500 \mathrm{MHz}\right) \delta 1.41(\mathrm{~s}, 9 \mathrm{H}, \mathrm{Ot}$ - $\underline{\mathrm{Bu}}), 3.49(\mathrm{t}, J=$ $10.7 \mathrm{~Hz}, 1 \mathrm{H}, \mathrm{PhCH}), 3.50$ (t, $\left.J=9.4 \mathrm{~Hz}, 1 \mathrm{H}, \mathrm{NCH}_{2}\right), 3.61\left(\mathrm{~s}, 3 \mathrm{H}, \mathrm{OCH}_{3}\right), 3.72(\mathrm{td}, J=11.2,7.4$ $\left.\mathrm{Hz}, 1 \mathrm{H}, \mathrm{NC}_{2}\right), 4.05$ (dd, $\left.J=10.4,7.7 \mathrm{~Hz}, 1 \mathrm{H}, \mathrm{PhC} \underline{\mathrm{H}}\right), 4.29$ (d, J=9.2 Hz, 1H, NC프), 7.13-7.26 $(\mathrm{m}, 10 \mathrm{H}, \mathrm{Ph} \underline{\mathrm{H}}) ;{ }^{13} \mathrm{C} \mathbf{N M R}\left(\mathrm{CDCl}_{3}\right.$, rotational isomers, $\left.25{ }^{\circ} \mathrm{C}, 125.6 \mathrm{MHz}\right) \delta 28.2\left(\mathrm{CH}_{3}\right), 28.3$ $\left(\mathrm{CH}_{3}\right), 50.6(\mathrm{CH}), 51.8(\mathrm{CH}), 51.9(\mathrm{CH}), 52.1(\mathrm{CH}), 52.9\left(\mathrm{CH}_{2}\right), 53.7\left(\mathrm{CH}_{2}\right), 56.7(\mathrm{CH}), 57.4$ $(\mathrm{CH}), 66.1\left(\mathrm{CH}_{3}\right), 66.7\left(\mathrm{CH}_{3}\right), 80.2(\mathrm{C}), 80.4(\mathrm{C}), 127.2(\mathrm{CH}), 127.4(\mathrm{CH}), 127.5(\mathrm{CH}), 127.6$ $(\mathrm{CH}), 128.5(\mathrm{CH}), 128.6(\mathrm{CH}), 128.7(\mathrm{CH}), 137.5(\mathrm{C}), 137.6(\mathrm{C}), 137.7(\mathrm{C}), 153.2(\mathrm{C}), 153.9$ (C), 172.9 (C), 173.0 (C). HRMS-FAB (M+1) Calcd for $\mathrm{C}_{23} \mathrm{H}_{28} \mathrm{NO}_{4}$ : 382.2018; Found: 382.2016 .

2,5-Dimethyl-3,4-diphenyl-pyrrolidine-1-carboxylic Acid tert-Butyl Ester (107). The general lithiation procedure was followed using $102(0.055 \mathrm{~g}, 0.163 \mathrm{mmol})$ and MeOTf (0.061 $\mathrm{mL}, 0.539 \mathrm{mmol})$ as the electrophile. Purification by column chromatography (20:1 pet ether/EtOAc) gave $107(0.025 \mathrm{~g}, 43 \%,>95: 5 \mathrm{dr})$ as an oil. Major diastereomer: ${ }^{1} \mathbf{H}$ NMR $\left(\mathrm{CDCl}_{3}, 500 \mathrm{MHz}\right) \delta 1.37\left(\mathrm{~d}, J=5.1 \mathrm{~Hz}, 6 \mathrm{H}, \mathrm{C}_{3} \underline{\mathrm{CH}}\right), 1.50(\mathrm{~s}, 9 \mathrm{H}, \mathrm{Ot}-\underline{\mathrm{Bu}}), 2.88(\mathrm{~m}, 2 \mathrm{H}, \mathrm{PhC} \underline{\mathrm{H}})$,

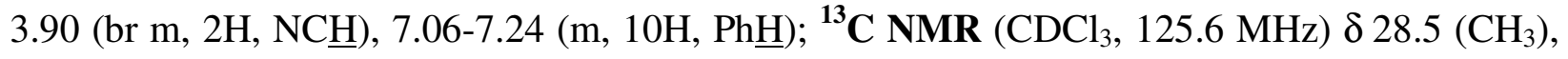
$60.2(\mathrm{CH}), 60.8(\mathrm{CH}), 79.2(\mathrm{C}), 126.8(\mathrm{CH}), 128.0(\mathrm{CH}), 128.4(\mathrm{CH}), 139.2(\mathrm{C}), 154.0(\mathrm{C})$. HRMS-FAB (M+1) Calcd for $\mathrm{C}_{23} \mathrm{H}_{30} \mathrm{NO}_{2}$ : 352.2277; Found: 352.2278.

\section{Representative Procedure for Boc-deprotection and Tosylation of Pyrrolidines: 2-} Methyl-3,4-diphenyl-1-(toluene-4-sulfonyl)-pyrrolidine. To a solution of $102(0.095 \mathrm{~g}, 0.282$ $\mathrm{mmol})$ in $\mathrm{CH}_{2} \mathrm{Cl}_{2}(7.8 \mathrm{~mL})$ was added TFA $(0.78 \mathrm{~mL})$ dropwise. The solution was stirred for $1 \mathrm{~h}$ at rt, carefully treated with sat. $\mathrm{NaHCO}_{3}(20 \mathrm{~mL})$ and stirred vigorously until gas evolution ceased. The aqueous was extracted with $\mathrm{CH}_{2} \mathrm{Cl}_{2}(3 \times 10 \mathrm{~mL})$ and the combined organics were dried over $\mathrm{Na}_{2} \mathrm{SO}_{4}$ and concentrated. The residue was redissolved in $\mathrm{CH}_{2} \mathrm{Cl}_{2}(2 \mathrm{~mL})$ and $\mathrm{Et}_{3} \mathrm{~N}$ 
(0.098 $\mathrm{mL}, 0.705 \mathrm{mmol})$ was added followed by tosyl chloride $(0.065 \mathrm{~g}, 0.338 \mathrm{mmol})$. The solution was stirred for $1 \mathrm{~h}$, diluted with $\mathrm{CH}_{2} \mathrm{Cl}_{2}(10 \mathrm{~mL})$ and washed with $0.1 \mathrm{~N} \mathrm{HCl}$. The aqueous was extracted with $\mathrm{CH}_{2} \mathrm{Cl}_{2}(3 \times 10 \mathrm{~mL})$ and the combined organics were dried over $\mathrm{MgSO}_{4}$ and concentrated. Purification by column chromatography (5:1 pet ether/EtOAc) gave the title compound $(0.088 \mathrm{~g}, 80 \%)$ as an oil. Crystals suitable for X-ray crystallographic analysis were obtained by addition of pentane: mp 100-101 ${ }^{\circ} \mathrm{C} .{ }^{1} \mathbf{H} \mathbf{N M R}\left(\mathrm{CDCl}_{3}, 500 \mathrm{MHz}\right) \delta 1.47(\mathrm{~d}, J$ $=6.0 \mathrm{~Hz}, 3 \mathrm{H}, \mathrm{CHMe}), 2.52\left(\mathrm{~s}, 3 \mathrm{H}, \mathrm{CH}_{3} \mathrm{Ph}\right), 2.86(\mathrm{td}, J=11.4,7.5 \mathrm{~Hz}, 1 \mathrm{H}, \mathrm{CHPh}), 2.99(\mathrm{dd}, J=$

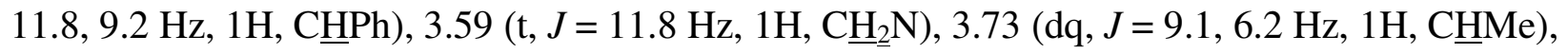
$4.04\left(\mathrm{dd}, J=12.0,7.5 \mathrm{~Hz}, 1 \mathrm{H}, \mathrm{CH}_{2} \mathrm{~N}\right), 6.86(\mathrm{~m}, 2 \mathrm{H}, \mathrm{Ph} \underline{\mathrm{H}}), 6.97(\mathrm{~m}, 2 \mathrm{H}, \mathrm{Ph} \underline{\mathrm{H}}), 7.11-7.21(\mathrm{~m}, 6$ $\mathrm{H}, \mathrm{Ph} \underline{\mathrm{H}}), 7.44(\mathrm{~d}, J=10.6 \mathrm{~Hz}, 2 \mathrm{H}, \mathrm{Ph} \underline{\mathrm{H}}), 7.77(\mathrm{~d}, J=8.2 \mathrm{~Hz}, 2 \mathrm{H}, \mathrm{Ph} \underline{\mathrm{H}}) ;{ }^{13} \mathrm{C} \mathbf{N M R}\left(\mathrm{CDCl}_{3}\right.$, $125.6 \mathrm{MHz}) \delta 20.6\left(\mathrm{CH}_{3}\right), 21.5\left(\mathrm{CH}_{3}\right), 50.2(\mathrm{CH}), 55.2\left(\mathrm{CH}_{2}\right), 60.9(\mathrm{CH}), 64.0(\mathrm{CH}), 127.0$ $(\mathrm{CH}), 127.1(\mathrm{CH}), 127.2(\mathrm{CH}), 127.5(\mathrm{CH}), 127.7(\mathrm{CH}), 128.4(\mathrm{CH}), 128.6(\mathrm{CH}), 129.8(\mathrm{CH})$, 135.2 (C), 137.8 (C), 138.3 (C), 143.6 (C). Anal. Calcd for $\mathrm{C}_{24} \mathrm{H}_{25} \mathrm{NO}_{2} \mathrm{~S}: \mathrm{C}, 73.62 ; \mathrm{H}, 6.44 ; \mathrm{N}$, 3.58. Found C, 73.59; H, 6.45; N, 3.74.

Octahydro-isoindole-2-carboxylic Acid tert-Butyl Ester (108). A solution of 109 (5.93 $\mathrm{g}, 26.6 \mathrm{mmol})$ in EtOAc $(150 \mathrm{~mL})$ was hydrogenated at atmospheric pressure for $6 \mathrm{~h}$ with $\mathrm{Pd} / \mathrm{C}$ (0.900 g). Filtration of the catalyst and concentration provided $\mathbf{1 0 8}(5.98 \mathrm{~g}, 99 \%)$ as an oil. ${ }^{1} \mathbf{H}$ NMR $\left(\mathrm{CDCl}_{3}, 500 \mathrm{MHz}\right) \delta 1.44(\mathrm{~s}, 9 \mathrm{H}, \mathrm{O} t-\underline{\mathrm{Bu}}), 1.28-1.60\left(\mathrm{~m}, 8 \mathrm{H}\right.$, ring- $\left.\underline{\mathrm{H}}_{2}\right), 2.14(\mathrm{~m}, 2 \mathrm{H}$, $\left.\mathrm{CH}_{2} \mathrm{CHCH}_{2}\right), 3.17\left(\mathrm{dd}, J=10.4,5.4 \mathrm{~Hz}, 2 \mathrm{H}, \mathrm{NC}_{2}\right), 3.28\left(\mathrm{dd}, J=10.7,7.0 \mathrm{~Hz}, 2 \mathrm{H}, \mathrm{NC}_{2}\right) ;{ }^{13} \mathrm{C}$ NMR $\left(\mathrm{CDCl}_{3}, 125.6 \mathrm{MHz}\right) \delta 22.7\left(\mathrm{CH}_{2}\right), 25.8\left(\mathrm{CH}_{2}\right), 28.5\left(\mathrm{CH}_{3}\right), 36.9(\mathrm{CH}), 49.7\left(\mathrm{CH}_{2}\right), 78.8$ (C), 155.2 (C). HRMS-FAB (M+1) Calcd for $\mathrm{C}_{13} \mathrm{H}_{24} \mathrm{NO}_{2}$ : 226.1807; Found: 226.1808.

1,3,3a,4,7,7a-Hexahydro-isoindole-2-carboxylic Acid tert-Butyl Ester (109). To a mixture of LAH $(6.27 \mathrm{~g}, 165 \mathrm{mmol})$ in THF $(50 \mathrm{~mL})$ was added a solution of 1,2,3,6tetrahydropthalamide $(10.0 \mathrm{~g}, 66.1 \mathrm{mmol})$ in THF $(150 \mathrm{~mL})$ carefully dropwise. The green mixture was refluxed for $24 \mathrm{~h}$ after complete addition, cooled to $\mathrm{rt}$ and quenched carefully with sat. $\mathrm{Na}_{2} \mathrm{SO}_{4}$. Excess $\mathrm{Na}_{2} \mathrm{SO}_{4}$ solid was added and the mixture filtered and concentrated to an orange oil. The oil was redissolved in $\mathrm{CH}_{2} \mathrm{Cl}_{2}(150 \mathrm{~mL})$ and a solution of Boc2O (15.2 mL, 66.1 mmol) in $\mathrm{CH}_{2} \mathrm{Cl}_{2}(20 \mathrm{~mL})$ was added dropwise. The resulting red solution was stirred for $1 \mathrm{~h}$ and concentrated. Purification by column chromatography (9:1 pet ether/EtOAc) and short-path 
distillation provided $109(10.8 \mathrm{~g}, 73 \%)$ as an oil. ${ }^{1} \mathbf{H} \mathbf{N M R}\left(\mathrm{CDCl}_{3}, 500 \mathrm{MHz}\right) \delta 1.42(\mathrm{~s}, 9 \mathrm{H}, \mathrm{O} t$ $\underline{\mathrm{Bu}}), 1.87\left(\mathrm{dd}, J=6.3,3.7 \mathrm{~Hz}, 2 \mathrm{H}, \mathrm{CH}_{2} \mathrm{CHCH}_{2}\right), 2.22\left(\mathrm{~m}, 4 \mathrm{H}, \mathrm{CH}=\mathrm{CHCH}_{2}\right), 3.09$ (dd, $J=10.3$, $\left.5.2 \mathrm{~Hz}, 2 \mathrm{H}, \mathrm{NCH}_{2}\right), 3.37\left(\mathrm{dd}, J=10.5,6.5 \mathrm{~Hz}, 2 \mathrm{H}, \mathrm{NCH}_{2}\right), 5.60(\mathrm{~s}, 2 \mathrm{H}, \mathrm{CH}=\mathrm{CH}) ;{ }^{13} \mathbf{C} \mathbf{N M R}$ $\left(\mathrm{CDCl}_{3}, 125.6 \mathrm{MHz}\right) \delta 24.6\left(\mathrm{CH}_{2}\right), 28.4\left(\mathrm{CH}_{3}\right), 33.7(\mathrm{CH}), 50.8\left(\mathrm{CH}_{2}\right), 78.8(\mathrm{C}), 124.3(\mathrm{CH})$, 155.2 (C). Anal. Calcd for $\mathrm{C}_{13} \mathrm{H}_{21} \mathrm{NO}_{2}$ : C, 69.92; H, 9.48; N, 6.27. Found C, 69.73; H, 9.51; N, 6.41 .

3,4-Diphenyl-pyrrolidine-1-carboxylic Acid tert-Butyl Ester (110). A solution of cis1-benzyl-3,4-diphenyl-pyrrolidine (1.75 g, $5.58 \mathrm{mmol}$ ) and $\mathrm{Boc}_{2} \mathrm{O}$ (2.56 mL, $\left.11.2 \mathrm{mmol}\right)$ in EtOAc $(62 \mathrm{~mL})$ was hydrogenated at $100 \mathrm{psi}$ for $14 \mathrm{~h}$ with $\mathrm{Pd}(\mathrm{OH})_{2}(0.600 \mathrm{~g})$. Filtration of the catalyst and concentration provided the crude product which was purified by column chromatography (9:1 pet ether/EtOAc) to provide $110(1.51 \mathrm{~g}, 78 \%)$ as a white solid: mp 97-98 ${ }^{\circ} \mathrm{C} .{ }^{1} \mathbf{H}$ NMR $\left(\mathrm{CDCl}_{3}, 500 \mathrm{MHz}\right) \delta 1.56\left(\mathrm{~s}, 9 \mathrm{H}, \mathrm{C}\left(\mathrm{C}_{3}\right)_{3}\right), 3.66(\mathrm{~m}, 2 \mathrm{H}, \mathrm{C} \underline{\mathrm{HPh}}),, 3.82(\mathrm{~m}, 4 \mathrm{H}$, $\left.\mathrm{NC}_{2}\right), 6.77(\mathrm{~m}, 4 \mathrm{H}, \mathrm{PhH}), 7.12(\mathrm{~m}, 6 \mathrm{H}, \mathrm{PhH}) ;{ }^{13} \mathbf{C} \mathbf{N M R}\left(\mathrm{CDCl}_{3}, 125.6 \mathrm{MHz}\right) \delta 28.5\left(\mathrm{CH}_{3}\right)$, $48.4(\mathrm{CH}), 49.2(\mathrm{CH}), 49.8\left(\mathrm{CH}_{2}\right), 50.3\left(\mathrm{CH}_{2}\right), 79.5(\mathrm{C}), 126.5(\mathrm{CH}), 127.9(\mathrm{CH}), 128.2(\mathrm{CH})$, 138.6 (C), 154.6 (C). Anal. Calcd for $\mathrm{C}_{21} \mathrm{H}_{25} \mathrm{NO}_{2}$ : C, 77.98; H, 7.79; N, 4.33. Found C, 78.05; H, $8.04 ; \mathrm{N}, 4.56$.

\section{Representative Procedure for Asymmetric Deprotonation of meso-Boc-3,4-} disubstituted Pyrrolidines: 1-Trimethylsilanyl-octahydro-isoindole-2-carboxylic Acid tert-

Butyl Ester (111). To a stirring solution of $108(0.267 \mathrm{~g}, 1.18 \mathrm{mmol})$ in ether $(7.5 \mathrm{~mL})-78{ }^{\circ} \mathrm{C}$ under $\mathrm{N}_{2}$ was added a precooled solution of $1(0.708 \mathrm{~mL}, 3.08 \mathrm{mmol})$ and $s e c-\mathrm{BuLi}(2.42 \mathrm{~mL}$ of a $1.27 \mathrm{M}$ solution in cyclohexane, $0.512 \mathrm{mmol})$ in ether $(7.5 \mathrm{~mL})$ at $-78{ }^{\circ} \mathrm{C}$ via a cold transfer flask. The solution was stirred for $3.5 \mathrm{~h}$ at $-78{ }^{\circ} \mathrm{C}$ and $\mathrm{TMSCl}(0.494 \mathrm{~mL}, 3.89 \mathrm{mmol})$ was added and the solution was stirred for an addition $1 \mathrm{~h}$ at $-78{ }^{\circ} \mathrm{C}$, warmed to $\mathrm{rt}$ and quenched with $\mathrm{H}_{2} \mathrm{O}(20 \mathrm{~mL})$. The aqueous was extracted with ether $(3 \times 20 \mathrm{~mL})$ and dried over $\mathrm{MgSO}_{4}$. Purification by column chromatography (10:1 pet ether/EtOAc) gave $111(0.176 \mathrm{~g}, 50 \%$, >95:5 dr) as a colorless oil. Major diastereomer: ${ }^{1} \mathbf{H} \mathbf{~ N M R}\left(\mathrm{CDCl}_{3}, 500 \mathrm{MHz}\right) \delta 0.18(\mathrm{~s}, 9 \mathrm{H}$, $\left.\left(\mathrm{C}_{3}\right)_{3} \mathrm{Si}\right), 1.20-1.60\left(\mathrm{~m}, 8 \mathrm{H}\right.$, ring- $\left.\underline{\mathrm{H}}_{2}\right), 1.44(\mathrm{~s}, 9 \mathrm{H}, \mathrm{Ot}-\underline{\mathrm{Bu}}), 2.0(\mathrm{~m}, 1 \mathrm{H}$, ring- $\underline{\mathrm{H}}), 2.15(\mathrm{~m}, 1 \mathrm{H}$, ring- $\underline{\mathrm{H}}$ ), 3.00 (br m, 1H, $\mathrm{NC}_{2}$ ), 3.13 (dd, $J=10.7,7.7 \mathrm{~Hz}, 2 \mathrm{H}, \mathrm{NC}_{2}$ ), 3.34 (br m, 1H, $\left.\mathrm{NCH}_{2}\right) ;{ }^{13} \mathrm{C}$ NMR $\left(\mathrm{CDCl}_{3}, 125.6 \mathrm{MHz}\right) \delta$-1.9 $\left(\mathrm{CH}_{3}\right), 21.6\left(\mathrm{CH}_{2}\right), 23.6\left(\mathrm{CH}_{2}\right), 25.3\left(\mathrm{CH}_{2}\right), 28.5$ 
$\left(\mathrm{CH}_{3}\right), 39.5(\mathrm{CH})$. Anal. Calcd for $\mathrm{C}_{16} \mathrm{H}_{31} \mathrm{NO}_{2} \mathrm{Si}$ : C, 64.59; H, 10.50; N, 4.71. Found C, 64.40; H, 10.37; N, 4.71. $[\alpha]_{\mathrm{D}}^{20}:-0.5^{\circ}\left(c=0.35, \mathrm{CHCl}_{3}\right)$.

\section{1-Trimethylsilanyl-1,3,3a,4,7,7a-hexahydro-isoindole-2-carboxylic Acid tert-Butyl}

Ester (112). The general asymmetric deprotonation procedure was followed using $109(0.589 \mathrm{~g}$, $2.64 \mathrm{mmol})$ and $\mathrm{TMSCl}(1.10 \mathrm{~mL}, 8.70 \mathrm{mmol})$ as the electrophile. Purification by column chromatography (20:1 pet ether/EtOAc) gave $112(0.375 \mathrm{~g}, 48 \%,>95: 5 \mathrm{dr})$ as an oil. Major

diastereomer: ${ }^{1} \mathbf{H}$ NMR $\left(\mathrm{CDCl}_{3}\right.$, rotational isomers, $\left.500 \mathrm{MHz}\right) \delta 0.04\left(\mathrm{~s}, 9 \mathrm{H},\left(\mathrm{C}_{\underline{3}}\right)_{3} \mathrm{Si}\right), 1.43$ (s, 9H, Ot- $\underline{\mathrm{Bu}}), 1.84\left(\mathrm{~m}, 2 \mathrm{H}, \mathrm{CH}=\mathrm{CHC}_{2}\right), 2.15\left(\mathrm{~m}, 4 \mathrm{H}, \mathrm{CH}=\mathrm{CHCH}_{2}, \mathrm{CH}=\mathrm{CHCH}_{2} \mathrm{C} \underline{\mathrm{H}}\right), 2.98(\mathrm{~d}$,

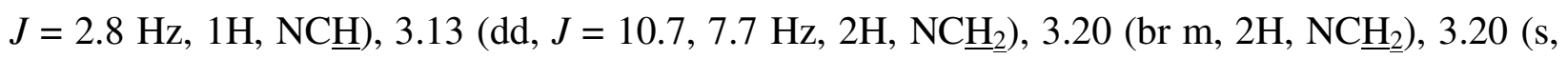
$\left.2 \mathrm{H}, \mathrm{CH}=\mathrm{CHCH}_{2}\right) ;{ }^{13} \mathrm{C} \mathbf{~ N M R}\left(\mathrm{CDCl}_{3}\right.$, rotational isomers, $\left.125.6 \mathrm{MHz}\right) \delta-1.8\left(\mathrm{CH}_{3}\right), 24.3\left(\mathrm{CH}_{2}\right)$, $24.5\left(\mathrm{CH}_{2}\right), 26.9\left(\mathrm{CH}_{2}\right), 27.1\left(\mathrm{CH}_{2}\right), 28.5\left(\mathrm{CH}_{3}\right), 28.6\left(\mathrm{CH}_{3}\right), 33.6(\mathrm{CH}), 34.4(\mathrm{CH}), 35.8(\mathrm{CH})$, $36.5(\mathrm{CH}), 50.6\left(\mathrm{CH}_{2}\right), 51.0\left(\mathrm{CH}_{2}\right), 54.4(\mathrm{CH}), 55.5(\mathrm{CH}), 78.4(\mathrm{C}), 79.1(\mathrm{C}), 78.4(\mathrm{C}), 124.1$ $(\mathrm{CH}), 124.2(\mathrm{CH}), 124.5(\mathrm{CH}), 124.7(\mathrm{CH}), 155.2(\mathrm{C}), 155.8(\mathrm{C})$. Anal. Calcd for $\mathrm{C}_{16} \mathrm{H}_{29} \mathrm{NO}_{2} \mathrm{Si}$ : C, 65.03; H, 9.89; N, 4.74. Found C, 64.73; H, 10.01; N, 4.86.

\section{3,4-Diphenyl-2-trimethylsilanyl-pyrrolidine-1-carboxylic Acid tert-Butyl Ester (113).}

The general asymmetric deprotonation procedure was followed using 110 (0.100 g, $0.309 \mathrm{mmol})$ and TMSCl $(0.129 \mathrm{~mL}, 1.02 \mathrm{mmol})$ as the electrophile. Purification by column chromatography (19:1 pet ether/EtOAc) gave $\mathbf{1 1 3}(0.056 \mathrm{~g}, 46 \%, 85: 15 \mathrm{dr})$ as a white solid: $\mathrm{mp} 72-74{ }^{\circ} \mathrm{C}$. Major diastereomer: ${ }^{1} \mathbf{H} \mathbf{N M R}\left(\mathrm{CDCl}_{3}\right.$, rotational isomers, $\left.500 \mathrm{MHz}\right) \delta 0.15\left(\mathrm{~s}, 9 \mathrm{H},\left(\mathrm{C}_{\underline{H}_{3}}\right)_{3} \mathrm{Si}\right)$, 1.57 (s, 9H, Ot- $\underline{\mathrm{Bu}}$ ), 3.60 (m, 3H, $\left.\mathrm{PhC} \underline{\mathrm{H}}, \mathrm{NC}_{2}\right), 3.79$ (d, $\left.J=4.3 \mathrm{~Hz}, 1 \mathrm{H}, \mathrm{NC} \underline{\mathrm{H}}\right), 3.90$ (m, $1 \mathrm{H}$, $\left.\mathrm{NC}_{\underline{H}}\right), 6.74(\mathrm{~m}, 4 \mathrm{H}, \mathrm{Ph} \underline{\mathrm{H}}), 7.09(\mathrm{~m}, 6 \mathrm{H}, \mathrm{Ph} \underline{\mathrm{H}}) ;{ }^{13} \mathbf{C} \mathbf{~ N M R}\left(\mathrm{CDCl}_{3}\right.$, rotational isomers, 125.6 MHz) $\delta$-1.8 $\left(\mathrm{CH}_{3}\right), 28.6\left(\mathrm{CH}_{3}\right), 48.7(\mathrm{CH}), 49.3\left(\mathrm{CH}_{2}\right), 49.8(\mathrm{CH}), 50.9\left(\mathrm{CH}_{2}\right), 51.8(\mathrm{CH}), 52.1$ $(\mathrm{CH}), 55.0(\mathrm{CH}), 126.3(\mathrm{CH}), 126.5(\mathrm{CH}), 127.8(\mathrm{CH}), 128.0(\mathrm{CH}), 128.1(\mathrm{CH}), 128.2(\mathrm{CH})$, 138.0 (C), 138.8 (C), 140.2 (C), 141.5 (C), 151.9 (C). Anal. Calcd for $\mathrm{C}_{24} \mathrm{H}_{33} \mathrm{NO}_{2} \mathrm{Si}$ : C, 72.86; $\mathrm{H}, 8.41 ; \mathrm{N}, 3.54$. Found $\mathrm{C}, 72.67 ; \mathrm{H}, 8.47 ; \mathrm{N}, 3.76$. The enantiomeric ratio of the major diastereomer was determined by CSP-HPLC on a Whelk-O column with $7.5 \%$ IPA/hexanes mobile phase and a flow rate of $1.0 \mathrm{~mL} / \mathrm{min}: \mathrm{rt}=4.20 \mathrm{~min}(58 \%), 5.40 \mathrm{~min}(42 \%)$. 
2-(Toluene-4-sulfonyl)-1-trimethylsilanyl-octahydro-isoindole. The general procedure for Boc-deprotection and tosylation of pyrrolidines was followed using 111 ( $0.170 \mathrm{~g}, 0.571$ mmol). Purification by column chromatography (5:1 pet ether/EtOAc) gave the title compound $(0.164 \mathrm{~g}, 82 \%)$ as a white solid. ${ }^{1} \mathbf{H}$ NMR $\left(\mathrm{CDCl}_{3}, 500 \mathrm{MHz}\right) \delta 0.11\left(\mathrm{~s}, 9 \mathrm{H},\left(\mathrm{CH}_{3}\right)_{3} \mathrm{Si}\right), 0.96(\mathrm{~m}$, $2 \mathrm{H}$, ring- $\left.\mathrm{C}_{2}\right), 1.07\left(\mathrm{~m}, 1 \mathrm{H}\right.$, ring- $\left.\underline{\mathrm{C}}_{2}\right), 1.26\left(\mathrm{~m}, 3 \mathrm{H}\right.$, ring- $\left.\mathrm{C}_{2}\right), 1.38\left(\mathrm{~m}, 2 \mathrm{H}\right.$, ring- $\left.\underline{\mathrm{C}}_{2}\right), 1.88$ (dtd, $J=11.1,5.6,1.5 \mathrm{~Hz}, 1 \mathrm{H}$, ring- $\mathrm{C} \underline{\mathrm{H}}), 2.28(\mathrm{~m}, 1 \mathrm{H}$, ring- $\underline{\mathrm{H}}), 2.41$ (s, $\left.3 \mathrm{H}, \mathrm{C}_{3} \underline{\mathrm{Ph}}\right), 2.78$ (d, $J$ $=1.8 \mathrm{~Hz}, 1 \mathrm{H}, \mathrm{NC} \underline{\mathrm{H}}), 3.06\left(\mathrm{t}, J=9.9 \mathrm{~Hz}, 1 \mathrm{H}, \mathrm{NC}_{2}\right), 3.26\left(\mathrm{t}, J=8.6 \mathrm{~Hz}, 1 \mathrm{H}, \mathrm{NC}_{2}\right), 7.29(\mathrm{~d}, J=$ $8.2 \mathrm{~Hz}, 2 \mathrm{H}, \mathrm{Ph} \underline{\mathrm{H}}), 7.71(\mathrm{~d}, J=8.2 \mathrm{~Hz}, 2 \mathrm{H}, \mathrm{Ph} \underline{\mathrm{H}}) ;{ }^{13} \mathbf{C} \mathbf{N M R}\left(\mathrm{CDCl}_{3}, 125.6 \mathrm{MHz}\right) \delta-2.1\left(\mathrm{CH}_{3}\right)$, $20.8\left(\mathrm{CH}_{2}\right), 21.4(\mathrm{CH}), 24.3\left(\mathrm{CH}_{2}\right), 24.5\left(\mathrm{CH}_{2}\right), 28.4\left(\mathrm{CH}_{2}\right), 36.9(\mathrm{CH}), 40.3\left(\mathrm{CH}_{3}\right), 50.1\left(\mathrm{CH}_{2}\right)$, $58.5(\mathrm{CH}), 127.8(\mathrm{CH}), 129.3(\mathrm{CH}), 133.9(\mathrm{C}), 143.0(\mathrm{C})$. Anal. Calcd for $\mathrm{C}_{18} \mathrm{H}_{29} \mathrm{NO}_{2} \mathrm{SSi}: \mathrm{C}$, $61.49 ; \mathrm{H}, 8.31 ; \mathrm{N}, 3.98$. Found $\mathrm{C}, 61.42 ; \mathrm{H}, 8.37 ; \mathrm{N}, 4.05$. The enantiomeric ratio of the major diastereomer was determined by CSP-HPLC on a Whelk-O column with $5 \%$ IPA/hexanes mobile phase and a flow rate of $2.0 \mathrm{~mL} / \mathrm{min}: \mathrm{rt}=6.26 \mathrm{~min}(87 \%), 8.57 \mathrm{~min}(13 \%)$. Recrystallization from pet ether provided the title compound ( $0.065 \mathrm{~g}, 33 \%, 97: 3 \mathrm{er}$ ). Crystals suitable for X-ray crystallographic analysis were obtained by vapor diffusion with pentane/ether: mp $120-121{ }^{\circ} \mathrm{C}$.

2-(Toluene-4-sulfonyl)-1-trimethylsilanyl-octahydro-isoindole (from 112). A solution of $112(0.109 \mathrm{~g}, 0.368 \mathrm{mmol})$ in EtOAc $(5 \mathrm{~mL})$ was hydrogenated at atmospheric pressure for 2 $\mathrm{h}$ with $\mathrm{Pd} / \mathrm{C}(0.025 \mathrm{~g})$. Filtration of the catalyst and concentration provided the crude in which the general procedure for Boc-deprotection and tosylation of pyrrolidines was followed. Purification by column chromatography (5:1 pet ether/EtOAc) gave the title compound $(0.101 \mathrm{~g}$, $82 \%$ ) which was spectroscopically identical to the compound derived from 111. The enantiomeric ratio of the major diastereomer was determined by CSP-HPLC on a Whelk-O column with $5 \% \mathrm{IPA} / \mathrm{h}$ exanes mobile phase and a flow rate of $2.0 \mathrm{~mL} / \mathrm{min}: \mathrm{rt}=7.34 \mathrm{~min}(73$ $\%), 10.60 \min (27 \%)$.

Representative Procedure for Transmetalation of Enantioenriched Stannanes and Conjugate Addition to Nitroalkenes: (4-Methoxy-phenyl)-(5-nitro-3,4-diphenyl-pent-1enyl)-carbamic Acid tert-Butyl Ester (ent-17). To a stirring solution of $\mathbf{1 1 4}^{8}(0.310 \mathrm{~g}, 0.617$ mmol) in toluene $(13 \mathrm{~mL})$ under $\mathrm{N}_{2}$ was added $1(0.170 \mathrm{~mL}, 0.741 \mathrm{mmol})$. The solution was 
cooled to $-78{ }^{\circ} \mathrm{C}$ and $n$-BuLi $(0.511 \mathrm{~mL}$ of a $1.45 \mathrm{M}$ solution in hexanes, $0.741 \mathrm{mmol})$ was added. The yellow solution was stirred for $1 \mathrm{~h}$ at $-78{ }^{\circ} \mathrm{C}$ and $10(0.120 \mathrm{~g}, 0.802 \mathrm{mmol})$ in toluene ( $3 \mathrm{~mL}$ ) was added dropwise over $1 \mathrm{~h}$ by syringe pump. After complete addition, the solution was stirred for an additional $10 \mathrm{~min}$ at $-78{ }^{\circ} \mathrm{C}$, quenched with $\mathrm{MeOH}(1 \mathrm{~mL})$ and warmed to rt. The solution was poured into water $(10 \mathrm{~mL})$ and brine $(5 \mathrm{~mL})$ and extracted with ether $(3 \mathrm{x} 15 \mathrm{~mL})$. The combined organics were dried over $\mathrm{MgSO}_{4}$ and concentrated to an orange oil. Purification by silica gel column chromatography (3:1 pet ether/EtOAc) gave ent-17 (0.178 g, $59 \%$, 85:15 dr) as a colorless oil. The ${ }^{1} \mathrm{H}$ NMR and ${ }^{13} \mathrm{C}$ NMR spectra were consistent with reported literature values. ${ }^{9}$ The enantiomeric ratio of the major diastereomer was determined by CSP-HPLC on a Whelk-O column with $10 \%$ IPA/hexanes mobile phase and a flow rate of $1.0 \mathrm{~mL} / \mathrm{min}: \mathrm{rt}=23.3$ $\min (88 \%), 29.6 \min (12 \%)$.

Transmetalation of 115. The general transmetalation procedure was followed using $\mathbf{1 1 5}^{9}$ (0.600 g, $\left.1.19 \mathrm{mmol}\right)$ and $10(0.235 \mathrm{~g}, 0.1 .55 \mathrm{mmol})$. Purification by silica gel column chromatography (3:1 pet ether/EtOAc) gave ent-17 $(0.489 \mathrm{~g}, 84 \%, 85: 15 \mathrm{dr})$ as a colorless oil. The enantiomeric ratio of the major diastereomer was determined by CSP-HPLC on a Whelk-O column with $10 \%$ IPA/hexanes mobile phase and a flow rate of $1.0 \mathrm{~mL} / \mathrm{min}: \mathrm{rt}=23.3 \mathrm{~min}(85$ $\%), 29.6 \min (15 \%)$.

\section{Representative Procedure for Enantiomeric Purity Determination of 2-Piperidones}

by Reduction and Acylation: 3-Hydroxy-4-phenyl-piperidine-1-carboxylic Acid (-)Menthyl Ester (116). To a solution of 57 (0.275 g, $0.792 \mathrm{mmol})$ in THF (14 mL) was added lithium aluminum hydride $(2.38 \mathrm{~mL}$ of a $1 \mathrm{M}$ solution in THF, $2.38 \mathrm{mmol})$ and the solution was reluxed for $4 \mathrm{~h}$. The solution was cooled to rt and saturated $\mathrm{Na}_{2} \mathrm{SO}_{4}(1 \mathrm{~mL})$ was added cautiously. Excess $\mathrm{Na}_{2} \mathrm{SO}_{4}$ solid was added and the solid was filtered off, washed with ether $(2 \times 25 \mathrm{~mL})$ and the filtrate was concentrated. The residue was redissolved in $\mathrm{CH}_{2} \mathrm{Cl}_{2}(7 \mathrm{~mL})$ and $\mathrm{Et}_{3} \mathrm{~N}(0.164$ $\mathrm{mL}, 1.18 \mathrm{mmol})$ and (-)-menthylchloroformate $(0.170 \mathrm{~mL}, 0.792 \mathrm{mmol})$ were added. The solution was stirred for $14 \mathrm{~h}$ and then poured into $\mathrm{H}_{2} \mathrm{O}(10 \mathrm{~mL})$, extracted with $\mathrm{CH}_{2} \mathrm{Cl}_{2}(3 \times 10$ $\mathrm{mL}$ ). The combined organics were dried over $\mathrm{MgSO}_{4}$ and concentrated. Purfication by column chromatography (3:1 pet ether/EtOAc) gave $116(0.236 \mathrm{~g}, 83 \%,>97: 3 \mathrm{dr})$ as a colorless oil. GC: $\left(\mathrm{t}_{\mathrm{i}}=100{ }^{\circ} \mathrm{C} \mathrm{t}_{\mathrm{f}}=200{ }^{\circ} \mathrm{C}, 20{ }^{\circ} \mathrm{C} / \mathrm{min}\right) \mathrm{t}_{\mathrm{Rmajor}}=37.23 \mathrm{~min} ., \mathrm{t}_{\mathrm{Rminor}}=36.4 \mathrm{~min}$ Major diastereomer: ${ }^{1} \mathbf{H}$ NMR $\left(\mathrm{CDCl}_{3}, 500 \mathrm{MHz}\right) \delta 0.83\left(\mathrm{~d}, J=6.6 \mathrm{~Hz}, 3 \mathrm{H}, \mathrm{CH}_{3} \mathrm{CH}\right), 0.93\left(\mathrm{~m}, 1 \mathrm{H},\left(\mathrm{CH}_{3}\right)_{2} \mathrm{CH}\right), 0.93$ 
$\left(\mathrm{d}, J=6.8 \mathrm{~Hz}, 6 \mathrm{H},\left(\mathrm{CH}_{3}\right)_{2} \mathrm{CH}\right), 1.10(\mathrm{~m}, 1 \mathrm{H}, \operatorname{ring}-\mathrm{C} \underline{\mathrm{H}}), 1.41\left(\mathrm{~m}, 1 \mathrm{H}, \mathrm{NCHC}_{2}\right), 1.55(\mathrm{~m}, 1 \mathrm{H}$, ring- $\underline{\mathrm{H}}), 1.70\left(\mathrm{~m}, 2 \mathrm{H}\right.$, ring- $\left.\underline{\mathrm{CH}}_{2}\right), 1.83\left(\mathrm{~m}, 2 \mathrm{H}, \mathrm{NCHCH}_{2}\right.$, ring- $\left.\underline{\mathrm{H}}\right), 1.95$ (quintd, $J=6.7,4.5$ $\mathrm{Hz}, 1 \mathrm{H}$, ring- $\underline{\mathrm{H}}), 2.11(\mathrm{~m}, 1 \mathrm{H}$, ring- $\underline{\mathrm{H}}), 2.55(\mathrm{td}, J=10.2,3.9 \mathrm{~Hz}, 1 \mathrm{H}, \mathrm{C} \underline{\mathrm{HPh}}), 2.68$ (br t, $J=$ $11.1 \mathrm{~Hz}, 1 \mathrm{H}, \mathrm{NC}_{2}$ ), 2.68 (br t, $J=12.0 \mathrm{~Hz}, 1 \mathrm{H}, \mathrm{NCH}_{2}$ ), 3.69 (br m, 1H, $\mathrm{NC}_{2}$ ), 4.25 (br m,

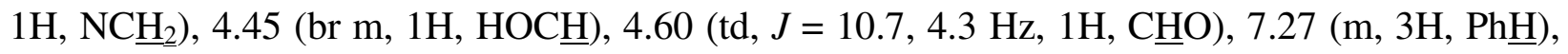
$7.36(\mathrm{~m}, 2 \mathrm{H}, \mathrm{Ph} \underline{\mathrm{H}}) ;{ }^{13} \mathrm{C} \mathbf{N M R}\left(\mathrm{CDCl}_{3}, 125.6 \mathrm{MHz}\right) \delta 16.6\left(\mathrm{CH}_{3}\right), 20.7(\mathrm{CH}), 22.0\left(\mathrm{CH}_{3}\right), 23.6$ $\left(\mathrm{CH}_{2}\right), 26.4(\mathrm{CH}), 31.3(\mathrm{CH}), 34.3\left(\mathrm{CH}_{2}\right), 41.5\left(\mathrm{CH}_{2}\right), 43.9\left(\mathrm{CH}_{2}\right), 47.3(\mathrm{CH}), 49.6\left(\mathrm{CH}_{2}\right), 51.4$ $(\mathrm{CH}), 70.8(\mathrm{CH}), 75.4(\mathrm{CH}), 127.2(\mathrm{CH}), 127.8(\mathrm{CH}), 128.9(\mathrm{CH}), 141.4(\mathrm{C}), 155.2(\mathrm{C})$. HRMSFAB (M+1): Calcd for $\mathrm{C}_{22} \mathrm{H}_{34} \mathrm{NO}_{3}$ : 360.2539; Found: 360.2538 .

1-Benzyl-3-hydroxy-4-phenyl-piperidine (118). To a solution of $72(0.20 \mathrm{~g}, 0.753$ mmol) in dry THF $(15 \mathrm{~mL})$ under a $\mathrm{N}_{2}$ atmosphere at $-78^{\circ} \mathrm{C}$ was added $t$ - BuLi $(0.53 \mathrm{~mL}$ of a 1.7 $\mathrm{M}$ solution in pentane, $0.90 \mathrm{mmol}$ ). The yellow solution was stirred for $30 \mathrm{~min}$ at $-78^{\circ} \mathrm{C}$, and added to $117(0.28 \mathrm{~g}, 0.90 \mathrm{mmol})$ in THF (5 mL) through inverse addition. The solution was stirred for $2.5 \mathrm{~h}$ at $-78^{\circ} \mathrm{C}$, warmed to rt, and quenched with sat. $\mathrm{Na}_{2} \mathrm{~S}_{2} \mathrm{O}_{3}$ solution $(10 \mathrm{~mL})$. The aqueous layer was extracted with $\mathrm{Et}_{2} \mathrm{O}(3 \times 20 \mathrm{~mL})$, dried over $\mathrm{MgSO}_{4}$, and concentrated. Filtration through a plug of silica $(2: 1$ pet ether/EtOAc) and concentration gave a residue which was dissolved in dry THF (12 mL). LAH was added $(2.36 \mathrm{~mL}$ of a $1 \mathrm{M}$ solution in THF, 2.15 $\mathrm{mmol}$ ) in one portion. The mixture was heated to reflux for $18 \mathrm{~h}$, cooled to $\mathrm{rt}$, and quenched with sat. $\mathrm{Na}_{2} \mathrm{SO}_{4}$ solution $(5 \mathrm{~mL})$. The organic layer was dried over $\mathrm{Na}_{2} \mathrm{SO}_{4}$ and concentrated. Purification by column chromatography (5:1 pet ether/EtOAc) gave $118(0.123 \mathrm{~g}, 61 \%)$ as a white solid: mp 90.5-92 ${ }^{\circ} \mathrm{C} .{ }^{1} \mathbf{H}$ NMR $\left(\mathrm{CDCl}_{3}, 500 \mathrm{MHz}\right) \delta 1.71-1.81\left(\mathrm{~m}, 2 \mathrm{H}, \mathrm{C}_{2} \mathrm{CH}_{2} \mathrm{~N}\right), 1.88$ (t, $\left.J=10.8 \mathrm{~Hz}, 1 \mathrm{H}, \mathrm{CH}_{2} \underline{\mathrm{CH}}_{2} \mathrm{~N}\right), 1.99\left(\mathrm{td}, J=11.4,3.2 \mathrm{~Hz}, 1 \mathrm{H}, \mathrm{CH}_{2} \underline{\mathrm{C}}_{2} \mathrm{~N}\right), 2.30(\mathrm{td}, J=10.5,4.7$ $\left.\mathrm{Hz}, 1 \mathrm{H}, \mathrm{CH}_{2} \mathrm{CH}(\mathrm{Ph})\right), 2.84\left(\mathrm{~d}, J=11.2 \mathrm{~Hz}, 1 \mathrm{H}, \mathrm{CH}(\mathrm{OH}) \underline{\mathrm{H}}_{2} \mathrm{~N}\right), 3.08(\mathrm{dd}, J=10.3,3.9 \mathrm{~Hz}, 1 \mathrm{H}$, $\left.\mathrm{CH}(\mathrm{OH}) \underline{\mathrm{H}}_{2} \mathrm{~N}\right), 3.53(\mathrm{q}, J=24.7,13.1 \mathrm{~Hz}, 2 \mathrm{H}, \mathrm{CH}(\mathrm{Ph})), 3.73(\mathrm{td}, J=9.9,4.3 \mathrm{~Hz}, 1 \mathrm{H}$, $\mathrm{C} \underline{\mathrm{H}}(\mathrm{OH})), 7.14-7.25(\mathrm{~m}, 10 \mathrm{H}, \mathrm{Ph} \underline{\mathrm{H}}) ;{ }^{13} \mathbf{C} \mathbf{N M R}\left(\mathrm{CDCl}_{3}, 125.7 \mathrm{MHz}\right) \delta 32.1\left(\mathrm{CH}_{2}\right), 51.5(\mathrm{CH})$, 53.6 $\left(\mathrm{CH}_{2}\right), 60.1\left(\mathrm{CH}_{2}\right), 63.1\left(\mathrm{CH}_{2}\right), 71.9(\mathrm{CH}), 127.1(\mathrm{CH}), 127.3(\mathrm{CH}), 128.2(\mathrm{CH}), 128.4$ (CH), $1328.9(\mathrm{CH}), 129.5(\mathrm{CH}), 138.1$ (C), 142.5 (C). Anal. Calcd for $\mathrm{C}_{18} \mathrm{H}_{12} \mathrm{NO}$ : C, 80.86; H, 7.92; N, 5.24. Found C, 80.85; H, 7.96; N, 5.29. 
1-Benzyl-2-oxo-4-phenyl-piperidine-3-carboxylic Acid Methyl Ester (119). The general procedure for $t$-BuLi enolization was followed using $72(0.378 \mathrm{~g}, 0.1 .42 \mathrm{mmol})$ and methyl chloroformate $(0.143 \mathrm{~mL}, 1.85 \mathrm{mmol})$. Purfication by column chromatography $(2: 1$ pet ether/EtOAc) gave the $119(0.416 \mathrm{~g}, 90 \%$, >95:5 dr) as a colorless oil. Major diastereomer: ${ }^{1} \mathbf{H}$ NMR (acetone- $\left.d_{6}, 500 \mathrm{MHz}\right) \delta 2.01\left(\mathrm{~m}, 1 \mathrm{H}, \mathrm{C}_{2}{ }_{2} \mathrm{CHPh}\right), 2.17(\mathrm{qd}, J=11.8,5.6 \mathrm{~Hz}, 1 \mathrm{H}$, $\left.\mathrm{C}_{2} \underline{\mathrm{CHPh}}\right), 3.35\left(\mathrm{~m}, 1 \mathrm{H}, \mathrm{CHCH}_{2} \mathrm{~N}\right), 3.40$ (m, 1H, $\left.\underline{\mathrm{HPh}}\right), 3.46\left(\mathrm{~m}, 1 \mathrm{H}, \mathrm{CHCH}_{2} \mathrm{~N}\right), 3.50$ (s, 3H, $\left.\mathrm{OCH}_{3}\right), 3.71\left(\mathrm{~d}, J=11.6 \mathrm{~Hz}, 1 \mathrm{H}, \mathrm{CHCO}_{2} \mathrm{Me}\right), 4.58\left(\mathrm{~d}, J=14.5 \mathrm{~Hz}, 1 \mathrm{H}, \mathrm{PhCH}_{2} \mathrm{~N}\right), 4.70(\mathrm{~d}, J=$ $\left.14.5 \mathrm{~Hz}, 1 \mathrm{H}, \mathrm{PhC}_{2} \underline{2} \mathrm{~N}\right), 7.26-7.38(\mathrm{~m}, 10 \mathrm{H}, \mathrm{Ph} \underline{\mathrm{H}}) ;{ }^{13} \mathrm{C}$ NMR (acetone-d $\left.d_{6}, 125.6 \mathrm{MHz}\right) \delta 29.8$ $\left(\mathrm{CH}_{2}\right), 43.5(\mathrm{CH}), 47.0\left(\mathrm{CH}_{2}\right), 50.1\left(\mathrm{CH}_{2}\right), 51.7(\mathrm{CH}), 57.1\left(\mathrm{CH}_{3}\right), 127.6(\mathrm{CH}), 127.7(\mathrm{CH})$, $127.9(\mathrm{CH}), 128.5(\mathrm{CH}), 129.2(\mathrm{CH}), 129.3(\mathrm{CH}), 138.1(\mathrm{C}), 143.0(\mathrm{C}), 166.0(\mathrm{C}), 170.9(\mathrm{C})$. HRMS-FAB $(\mathrm{M}+1)$ Calcd for $\mathrm{C}_{20} \mathrm{H}_{22} \mathrm{NO}$ : 324.1600; Found: 324.1600 .

\section{Conversion of 119 to 120.}

Part 1: (1-Benzyl-4-phenyl-piperidin-3-yl)-methanol. The general reduction procedure for benzyl lactams was followed using $119(0.260 \mathrm{~g}, 0.804 \mathrm{mmol})$ and lithium aluminum hydride ( $8.03 \mathrm{~mL}$ of a $1 \mathrm{M}$ solution in THF, $8.03 \mathrm{mmol}$ ) with a $6 \mathrm{~h}$ reflux period. Purification by column chromatography (EtOAc) provided the title compound $(0.168 \mathrm{~g}, 74 \%)$ as a colorless oil. Major diastereomer: ${ }^{1} \mathbf{H}$ NMR $\left(\mathrm{CDCl}_{3}, 400 \mathrm{MHz}\right) \delta 1.86\left(\mathrm{~m}, 2 \mathrm{H}, \mathrm{CHCH}_{2} \mathrm{O}, \mathrm{CH}_{2} \mathrm{CHPh}\right), 2.05(\mathrm{~m}, 3 \mathrm{H}$, $\left.\mathrm{CHCH}_{2} \underline{\mathrm{N}}_{2} \underline{\mathrm{CH}}_{2} \mathrm{CHPh}\right), 2.36\left(\mathrm{td}, J=11.2,3.4 \mathrm{~Hz}, 2 \mathrm{H}, \mathrm{CHC}_{2} \underline{\mathrm{N}}\right), 2.99(\mathrm{~d}, J=10.8 \mathrm{~Hz}, 1 \mathrm{H}$, $\left.\mathrm{CHCH}_{2} \mathrm{~N}\right), 3.23\left(\mathrm{~m}, 2 \mathrm{H}, \mathrm{OH}, \mathrm{CHCH}_{2} \mathrm{O}\right), 3.38\left(\mathrm{~d}, J=10.3 \mathrm{~Hz}, 1 \mathrm{H}, \mathrm{CHCH}_{2} \mathrm{O}\right), 3.57(\mathrm{~d}, J=12.9$ $\left.\mathrm{Hz}, 1 \mathrm{H}, \mathrm{PhCH}_{2} \mathrm{~N}\right), 3.66\left(\mathrm{~d}, J=12.9 \mathrm{~Hz}, 1 \mathrm{H}, \mathrm{PhCH}_{2} \mathrm{~N}\right), 7.20-7.43(\mathrm{~m}, 10 \mathrm{H}, \mathrm{Ph} \underline{\mathrm{H}}) ;{ }^{13} \mathbf{C} \mathbf{N M R}$ $\left(\mathrm{CDCl}_{3}, 100.6 \mathrm{MHz}\right) \delta 34.1\left(\mathrm{CH}_{2}\right), 43.8(\mathrm{CH}), 44.9(\mathrm{CH}), 53.7\left(\mathrm{CH}_{2}\right), 57.3\left(\mathrm{CH}_{2}\right), 63.4\left(\mathrm{CH}_{2}\right)$, $64.8\left(\mathrm{CH}_{2}\right), 126.3(\mathrm{CH}), 127.0(\mathrm{CH}), 127.4(\mathrm{CH}), 128.1(\mathrm{CH}), 128.4(\mathrm{CH}), 129.3(\mathrm{CH}), 137.7$ (C), 144.4 (C). HRMS-FAB (M+1) Calcd for $\mathrm{C}_{19} \mathrm{H}_{24} \mathrm{NO}$ : 282.1858; Found: 282.1859.

\section{Part 2: 3-Hydroxymethyl-4-phenyl-piperidine-1-carboxylic Acid tert-Butyl Ester}

(120). A solution of (1-benzyl-4-phenyl-piperidin-3-yl)-methanol $(0.165 \mathrm{~g}, 0.586 \mathrm{mmol})$ and $\operatorname{Boc}_{2} \mathrm{O}(0.269 \mathrm{~mL}, 1.17 \mathrm{mmol})$ in EtOAc $(15 \mathrm{~mL})$ was hydrogenated at atmospheric pressure overnight with $\mathrm{Pd}(\mathrm{OH})_{2}(0.100 \mathrm{~g})$. Filtration of the catalyst and concentration provided the crude product which was purified by column chromatography (3:1 pet ether/EtOAc) to provide 120 (0.149 g, $87 \%)$ as a white solid: $\mathrm{mp} 133-134{ }^{\circ} \mathrm{C}$. lit. ${ }^{10} \mathrm{mp} 132-133{ }^{\circ} \mathrm{C}$ The ${ }^{1} \mathrm{H}$ NMR and ${ }^{13} \mathrm{C}$ 
NMR spectra were consistent with reported literature values. ${ }^{10} \operatorname{Ref}[\alpha]_{\mathrm{D}}^{20}:+8.09^{\circ}(c=1.1$, $\mathrm{MeOH})$. lit. $^{10}[\alpha]_{\mathrm{D}}^{20}:+6.4^{\circ}(c=0.4, \mathrm{MeOH})$.

\section{Conversion of 120 to 121.}

Part 1: 3-(4-Methoxy-phenoxymethyl)-4-phenyl-piperidine-1-carboxylic Acid tertButyl Ester. To a solution of $120(0.075 \mathrm{~g}, 0.257 \mathrm{mmol})$ in $\mathrm{CH}_{2} \mathrm{Cl}_{2}(3.5 \mathrm{~mL})$ was added $\mathrm{Et}_{3} \mathrm{~N}$ $(0.054 \mathrm{~mL}, 0.386 \mathrm{mmol})$ followed by methanesulfonyl chloride $(0.030 \mathrm{~mL}, 0.386 \mathrm{~mL})$ at $\mathrm{rt}$. The solution was stirred for $10 \mathrm{~min}$ and poured into $\mathrm{H}_{2} \mathrm{O}(10 \mathrm{~mL})$. The aqueous was extracted with $\mathrm{CH}_{2} \mathrm{Cl}_{2}(3 \times 10 \mathrm{~mL})$ and the combined organics were dried over $\mathrm{MgSO}_{4}$ and concentrated to give the crude mesylate. To a solution of $p$-methoxyphenol $(0.064 \mathrm{~g}, 0.514 \mathrm{mmol})$ in DMF $(1.0 \mathrm{~mL})$ was added $\mathrm{NaH}(0.019 \mathrm{~g}, 0.771 \mathrm{mmol})$ and the mixture was stirred for $10 \mathrm{~min}$. To the mixture was added a solution of the mesylate in DMF $(2 \mathrm{~mL})$ and the reaction was stirred at $60{ }^{\circ} \mathrm{C}$ for 2 h. The reaction was then heated to reflux for 10 min, cooled to rt and quenced carefully with $\mathrm{H}_{2} \mathrm{O}$ $(1 \mathrm{~mL})$. The reaction was poured into $\mathrm{H}_{2} \mathrm{O}(10 \mathrm{~mL})$ and the aqueous was extracted with ether (3 x $15 \mathrm{~mL}$ ). The combined organics were dried over $\mathrm{MgSO}_{4}$ and concentrated. Purification by column chromatography (6:1 pet ether/EtOAc) provided the title compound $(0.074 \mathrm{~g}, 73 \%)$ as a colorless oil. The ${ }^{1} \mathrm{H}$ NMR and ${ }^{13} \mathrm{C}$ NMR spectra were consistent with reported literature values. $10[\alpha]_{\mathrm{D}}^{20}:+26.3^{\circ}(c=0.51, \mathrm{MeOH})$. lit. ${ }^{10}[\alpha]_{\mathrm{D}}^{20}:+22.9^{\circ}(c=0.26, \mathrm{MeOH})$.

Part 2: (+)-Femoxetine (121). The general reduction procedure for benzyl lactams was followed using 3-(4-methoxy-phenoxymethyl)-4-phenyl-piperidine-1-carboxylic acid tert-butyl ester $(0.045 \mathrm{~g}, 0.113 \mathrm{mmol})$ and lithium aluminum hydride $(0.565 \mathrm{~mL}$ of a $1 \mathrm{M}$ solution in THF, $0.565 \mathrm{mmol}$ ) stirring the reaction $4 \mathrm{~h}$ at $\mathrm{rt}$. Purification by column chromatography on basic alumina (3:1 pet ether/EtOAc) provided $83(0.024 \mathrm{~g}, 67 \%)$ as a colorless oil. The ${ }^{1} \mathrm{H}$ NMR and ${ }^{13} \mathrm{C}$ NMR spectra were consistent with reported literature values. ${ }^{10}[\alpha]_{\mathrm{D}}^{20}:+73.0^{\circ}(c=0.55$, $\mathrm{MeOH})$. lit. ${ }^{10}[\alpha]_{\mathrm{D}}^{20}:+75.7^{\circ}(c=0.6, \mathrm{MeOH})$.

3-iso-Butyl-4-methyl-pyrrolidine-1-carboxylic Acid (-)-Menthyl Ester. The general reduction and acylation procedure was followed using $64(0.120 \mathrm{~g}, 0.77 \mathrm{mmol})$. Purification by column chromatography (11:1 pet ether/EtOAc) gave the title compound $(0.212 \mathrm{~g}, 85 \%,>97: 3$ 
dr) as an oil. GC: $\left(t_{i}=100{ }^{\circ} \mathrm{Ct}_{\mathrm{f}}=200{ }^{\circ} \mathrm{C}, 20{ }^{\circ} \mathrm{C} / \mathrm{min}\right) \mathrm{t}_{\text {Rmajor }}=14.867 \mathrm{~min}$. Major diastereomer: ${ }^{1} \mathbf{H}$ NMR $\left(\mathrm{CDCl}_{3}, 500 \mathrm{MHz}\right) \delta 0.78\left(\mathrm{~d}, J=6.9 \mathrm{~Hz}, 3 \mathrm{H}, \mathrm{C}_{3} \underline{\mathrm{CH}}\right), 0.88(\mathrm{~m}, 12 \mathrm{H}$, $\left.\left(\mathrm{C}_{\underline{3}}\right)_{2} \mathrm{CHCH}_{2}\right), 0.99$ (d, $\left.J=6.4 \mathrm{~Hz}, 3 \mathrm{H}, \mathrm{C}_{\underline{3}} \underline{\mathrm{CH}}\right), 1.04$ (dd, $J=12.9,3.4 \mathrm{~Hz}, 1 \mathrm{H}$, ring-C $\left.\underline{H}\right), 1.11$ (ddd, $\left.J=13.6,10.3,4.7 \mathrm{~Hz}, 2 \mathrm{H},\left(\mathrm{CH}_{3}\right)_{2} \mathrm{CHCH}_{2}\right), 1.34\left(\mathrm{~m}, 2 \mathrm{H}\right.$, ring- $\left.\underline{\mathrm{H}}, \mathrm{CH}_{3} \mathrm{C} \underline{\mathrm{H}}\right), 1.47(\mathrm{~m}, 1 \mathrm{H}$, $\left.\left(\mathrm{CH}_{3}\right)_{2} \mathrm{CHCH}_{2}\right), 1.54\left(\mathrm{~m}, 1 \mathrm{H},\left(\mathrm{CH}_{3}\right)_{2} \mathrm{CHCH}_{2}\right), 1.70\left(\mathrm{~m}, 4 \mathrm{H}, \mathrm{CH}_{2} \mathrm{CHCH}_{2} \mathrm{~N}\right.$, ring-C $\left.\underline{H}\right), 1.93$ (septetd, $\left.J=13.9,2.8 \mathrm{~Hz}, 1 \mathrm{H}, \mathrm{CH}_{3} \mathrm{CH}\right), 2.06(\mathrm{~m} \mathrm{1H}$, ring- $\underline{\mathrm{H}}), 2.84\left(\mathrm{~m}, 2 \mathrm{H}, \mathrm{NC}_{2}\right), 3.61(\mathrm{~m}$, $\left.2 \mathrm{H}, \mathrm{NCH}_{2}\right), 4.52(\mathrm{td}, J=11.0,4.5 \mathrm{~Hz}, 1 \mathrm{H}, \mathrm{CHO}) ;{ }^{13} \mathbf{C} \mathbf{N M R}\left(\mathrm{CDCl}_{3}, 125.6 \mathrm{MHz}\right) \delta 15.8\left(\mathrm{CH}_{3}\right)$, $16.5\left(\mathrm{CH}_{3}\right), 20.8(\mathrm{CH}), 21.6\left(\mathrm{CH}_{3}\right), 23.5\left(\mathrm{CH}_{2}\right), 23.9(\mathrm{CH}), 26.2\left(\mathrm{CH}_{3}\right), 26.7\left(\mathrm{CH}_{3}\right), 31.3(\mathrm{CH})$, $34.3\left(\mathrm{CH}_{2}\right), 41.3\left(\mathrm{CH}_{2}\right), 41.8\left(\mathrm{CH}_{2}\right), 47.3(\mathrm{CH}), 51.9\left(\mathrm{CH}_{2}\right), 53.1\left(\mathrm{CH}_{2}\right), 74.4(\mathrm{CH}), 154.7(\mathrm{C})$. HRMS-FAB (M+1) Calcd for $\mathrm{C}_{20} \mathrm{H}_{38} \mathrm{NO}_{2}: 324.2903$; Found: 324.2902. $[\alpha]_{\mathrm{D}}^{20}:-128.1^{\circ}(c=1.6$, $\left.\mathrm{CHCl}_{3}\right)$.

3-Phenyl-pyrrolidine-1-carboxylic Acid (-)-Menthyl Ester. The general reduction and acylation procedure was followed using $65(0.150 \mathrm{~g}, 0.93 \mathrm{mmol})$. Purification by column chromatography (10:1 pet ether/EtOAc) gave the title compound $(0.230 \mathrm{~g}, 75 \%, 75: 25 \mathrm{dr})$ as an oil. Major diastereomer: ${ }^{1} \mathbf{H} \mathbf{N M R}\left(\mathrm{CDCl}_{3}, 500 \mathrm{MHz}\right) \delta 0.82\left(\mathrm{~d}, J=6.0 \mathrm{~Hz}, 3 \mathrm{H}, \mathrm{C}_{3} \underline{\mathrm{CH}}\right), 0.91$ $\left(\mathrm{d}, J=6.8 \mathrm{~Hz}, 6 \mathrm{H},\left(\mathrm{CH}_{3}\right)_{2} \mathrm{CH}\right), 0.97(\mathrm{~m}, 1 \mathrm{H}$, ring- $\underline{\mathrm{H}}), 1.08(\mathrm{~m}, 1 \mathrm{H}$, ring- $\mathrm{C} \underline{\mathrm{H}}), 1.36(\mathrm{~m}, 1 \mathrm{H}$, ring$\mathrm{C} \underline{\mathrm{H}}), 1.51\left(\mathrm{~m}, 2 \mathrm{H},\left(\mathrm{CH}_{3}\right)_{2} \mathrm{CH}\right), 1.68\left(\mathrm{~m}, 2 \mathrm{H}, \mathrm{NCHC}_{2}\right.$, ring- $\left.\underline{\mathrm{H}}\right), 1.97$ (m $2 \mathrm{H}, \mathrm{NCHC}_{2}$, ring$\mathrm{C} \underline{\mathrm{H}}), 2.12(\mathrm{~m}, 1 \mathrm{H}$, ring- $\underline{\mathrm{H}}), 2.26(\mathrm{~m}, 1 \mathrm{H}$, ring- $\underline{\mathrm{H}}), 3.37\left(\mathrm{~m}, 3 \mathrm{H}, \mathrm{PhC} \underline{\mathrm{H}}, \mathrm{NC}_{\underline{2}}\right), 3.64(\mathrm{~m}, 1 \mathrm{H}$, $\left.\mathrm{NC}_{2}\right), 3.87\left(\mathrm{~m}, 1 \mathrm{H}, \mathrm{NCH}_{2}\right), 4.58(\mathrm{td}, J=10.7,4.3 \mathrm{~Hz}, 1 \mathrm{H}, \mathrm{CHO}), 7.23(\mathrm{~m}, 3 \mathrm{H}, \mathrm{Ph} \underline{\mathrm{H}}), 7.34(\mathrm{~m}$,

$2 \mathrm{H}, \mathrm{PhH}) ;{ }^{13} \mathrm{C} \mathrm{NMR}\left(\mathrm{CDCl}_{3}, 125.6 \mathrm{MHz}\right) \delta 16.4\left(\mathrm{CH}_{3}\right), 20.7(\mathrm{CH}), 20.8(\mathrm{CH}), 22.0\left(\mathrm{CH}_{3}\right)$, $23.5\left(\mathrm{CH}_{2}\right), 26.2(\mathrm{CH}), 31.2(\mathrm{CH}), 32.1\left(\mathrm{CH}_{2}\right), 32.4\left(\mathrm{CH}_{2}\right), 33.1\left(\mathrm{CH}_{2}\right), 33.2\left(\mathrm{CH}_{2}\right), 34.3\left(\mathrm{CH}_{2}\right)$, $41.7\left(\mathrm{CH}_{2}\right), 43.2(\mathrm{CH}), 44.0(\mathrm{CH}), 44.1(\mathrm{CH}), 45.5\left(\mathrm{CH}_{2}\right), 45.8\left(\mathrm{CH}_{2}\right), 45.9\left(\mathrm{CH}_{2}\right), 47.2(\mathrm{CH})$, $47.3(\mathrm{CH} 2), 52.1\left(\mathrm{CH}_{2}\right), 74.6(\mathrm{CH}), 74.6(\mathrm{CH}), 126.7(\mathrm{CH}), 126.9(\mathrm{CH}), 128.5(\mathrm{CH}), 141.2(\mathrm{C})$, 141.2 (C), 141.2 (C), 154.7 (C), 154.8 (C). Anal. Calcd for $\mathrm{C}_{21} \mathrm{H}_{31} \mathrm{NO}_{2}$ : C, 76.55; H, 9.48; N, 4.25. Found C, 76.63; H, 9.74; N, 4.42 .

\section{3-(4-Methoxy-phenoxymethyl)-4-phenyl-piperidine-1-carboxylic Acid (-)-Menthyl}

Ester. To a solution of 3-(4-Methoxy-phenoxymethyl)-4-phenyl-piperidine-1-carboxylic acid tert-butyl ester $(0.074 \mathrm{~g}, 0.186 \mathrm{mmol})$ in $\mathrm{CH}_{2} \mathrm{Cl}_{2}(5 \mathrm{~mL})$ was added TFA $(0.500 \mathrm{~mL})$. TLC indicated no remaining starting material after 45 min of stirring. The reaction was washed with 
sat $\mathrm{NaHCO}_{3}(15 \mathrm{~mL})$ and the organics separated. The aqueous was extracted with $\mathrm{CH}_{2} \mathrm{Cl}_{2}(3 \mathrm{x}$ $15 \mathrm{~mL}$ ) and the combined organics were dried over $\mathrm{Na}_{2} \mathrm{SO}_{4}$ and concentrated. The residue was dissolved in dry $\mathrm{CH}_{2} \mathrm{Cl}_{2}(3.5 \mathrm{~mL})$ under and $\mathrm{N}_{2}$ atmosphere. $\mathrm{Et}_{3} \mathrm{~N}(0.028 \mathrm{~mL}, 0.203 \mathrm{mmol})$ was added followed by (-)-menthylchloroformate $(0.044 \mathrm{~mL}, 0.203 \mathrm{~mL})$ and the reaction was stirred for $16 \mathrm{~h}$. Concentration and purfication of the residue by column chromatography (4:1 pet ether/EtOAc) of the residue gave the title compound $(0.017 \mathrm{~g}, 80 \%,>97: 3 \mathrm{dr})$ as a colorless oil.

Major diastereomer: ${ }^{1} \mathrm{H}$ NMR $\left(\mathrm{CDCl}_{3}, 500 \mathrm{MHz}\right) \delta 0.85\left(\mathrm{~d}, J=6.6 \mathrm{~Hz}, 3 \mathrm{H}, \mathrm{C}_{3} \underline{\mathrm{CH}}\right), 0.90(\mathrm{~m}$, $1 \mathrm{H}$, ring- $\left.\underline{\mathrm{C}}_{2}\right), 0.93\left(\mathrm{~d}, J=6.4 \mathrm{~Hz}, 6 \mathrm{H},\left(\mathrm{C}_{3}\right)_{2} \mathrm{CH}\right), 0.94\left(\mathrm{~d}, J=6.4 \mathrm{~Hz}, 6 \mathrm{H},\left(\mathrm{C}_{\mathrm{H}_{3}}\right)_{2} \mathrm{CH}\right), 1.0(\mathrm{~m}$, $1 \mathrm{H}$, ring- $\underline{\mathrm{H}}), 1.11\left(\mathrm{qd}, J=12.8,3.2 \mathrm{~Hz}, 1 \mathrm{H}, \mathrm{NCHCH}_{2}\right), 1.42(\mathrm{t}, J=11.2 \mathrm{~Hz}, 1 \mathrm{H}$, ring-C $\underline{\mathrm{H}})$, $1.53\left(\mathrm{~m}, 1 \mathrm{H},\left(\mathrm{CH}_{3}\right)_{2} \mathrm{C} \underline{\mathrm{H}}\right), 1.70\left(\mathrm{~m}, 2 \mathrm{H}\right.$, ring- $\left.\underline{\mathrm{H}}_{2}\right), 1.80\left(\mathrm{~m}, 2 \mathrm{H}, \mathrm{NCHCH}_{2}\right.$, ring- $\left.\underline{\mathrm{H}}\right), 1.98(\mathrm{~m}$, $1 \mathrm{H}$, ring- $\underline{\mathrm{H}}), 2.12\left(\mathrm{~m}, 2 \mathrm{H}, \mathrm{CHCH}_{2} \mathrm{O}\right.$, ring- $\left.\underline{\mathrm{H}}\right), 2.68\left(\mathrm{~m}, 1 \mathrm{H}, \mathrm{NC}_{2}\right), 2.85(\mathrm{dd}, J=13.5,11.3$ $\left.\mathrm{Hz}, 1 \mathrm{H}, \mathrm{NC}_{2}\right), 2.88(\mathrm{~m}, 1 \mathrm{H}, \mathrm{CHPh}), 3.50\left(\mathrm{dd}, J=9.5,7.1 \mathrm{~Hz}, 1 \mathrm{H}, \mathrm{OC}_{2}\right), 3.65(\mathrm{dd}, J=9.5$, $\left.2.8 \mathrm{~Hz}, 1 \mathrm{H}, \mathrm{OC}_{2}\right), 3.73\left(\mathrm{~s}, 3 \mathrm{H}, \mathrm{OC}_{\underline{3}}\right), 4.32$ (br m, 1H, $\mathrm{NC}_{2}$ ), 4.55 (br m, 1H, $\left.\mathrm{NC}_{2}\right), 4.60$ $(\mathrm{td}, J=10.9,4.0 \mathrm{~Hz}, 1 \mathrm{H}, \mathrm{CHO}), 6.68(\mathrm{~m}, 2 \mathrm{H}, \mathrm{Ph} \underline{\mathrm{H}}), 6.76(\mathrm{~m}, 2 \mathrm{H}, \mathrm{Ph} \underline{\mathrm{H}}), 7.20(\mathrm{~m} \mathrm{3H}, \mathrm{Ph} \underline{\mathrm{H}}), 7.29$ $(\mathrm{m}, 2 \mathrm{H}, \mathrm{Ph} \underline{\mathrm{H}}) ;{ }^{13} \mathrm{C} \mathrm{NMR}\left(\mathrm{CDCl}_{3}, 125.6 \mathrm{MHz}\right) \delta 16.8\left(\mathrm{CH}_{3}\right), 20.2(\mathrm{CH}), 22.0\left(\mathrm{CH}_{3}\right), 23.8\left(\mathrm{CH}_{2}\right)$, $26.5(\mathrm{CH}), 31.3(\mathrm{CH}), 33.7\left(\mathrm{CH}_{2}\right), 34.3\left(\mathrm{CH}_{2}\right), 41.5\left(\mathrm{CH}_{2}\right), 44.3\left(\mathrm{CH}_{2}\right), 44.8(\mathrm{CH}), 47.3(\mathrm{CH})$, $47.4\left(\mathrm{CH}_{2}\right), 55.6\left(\mathrm{CH}_{3}\right), 68.5\left(\mathrm{CH}_{2}\right), 75.2(\mathrm{CH}), 114.4(\mathrm{CH}), 115.3(\mathrm{CH}), 126.7(\mathrm{CH}), 127.4$ (CH), $128.7(\mathrm{CH}), 143.4(\mathrm{C}), 152.9$ (C), 153.7 (C), 155.3 (C). HRMS-FAB (M+1): Calcd for $\mathrm{C}_{30} \mathrm{H}_{42} \mathrm{NO}_{4}$ : 480.3114; Found: 480.3115. $[\alpha]_{\mathrm{D}}^{20}:-18.8^{\circ}\left(c=0.85, \mathrm{CHCl}_{3}\right)$.

\section{3-(4-Bromo-phenyl)-1-(4-methoxy-phenyl)-1-(3-nitro-1,2-diphenyl-propyl)-urea.}

To a solution $36(0.200 \mathrm{~g})$ in $\mathrm{CH}_{2} \mathrm{Cl}_{2}(5 \mathrm{~mL})$ was added trifluoroacetic acid $(1 \mathrm{~mL})$ at room temperature. The reaction mixture was stirred for $2 \mathrm{~h}$. After evaporation of volatile materials under reduced pressure, the residue was dissolved in ether, washed with sat. $\mathrm{NaHCO}_{3}$, dried over $\mathrm{MgSO}_{4}$, filtered and concentrated in vacuo affording $0.148 \mathrm{~g}(84 \%)$ of the crude amine. The product was used for the next step without further purification: ${ }^{1} \mathbf{H} \mathbf{~ N M R}\left(400 \mathrm{MHz}, \mathrm{CDCl}_{3}\right) \delta$ $3.73(\mathrm{~s}, 3 \mathrm{H}), 3.96(\mathrm{~s}, 1 \mathrm{H}), 4.03(\mathrm{ddd}, J=6.0,8.2,8.3 \mathrm{~Hz}, 1 \mathrm{H}), 4.61$ (d, $J=8.0 \mathrm{~Hz}, 1 \mathrm{H}), 4.83$ (dd, $J=8.8,13.6 \mathrm{~Hz}, 1 \mathrm{H}), 5.08(\mathrm{dd}, J=6.0,13.2 \mathrm{~Hz}, 1 \mathrm{H}), 6.54-6.60(\mathrm{~m}, 2 \mathrm{H}), 6.70-6.77(\mathrm{~m}, 2 \mathrm{H})$, 7.00-7.08 $(\mathrm{m}, 4 \mathrm{H}), 7.14-7.30(\mathrm{~m}, 6 \mathrm{H})$. The crude amine and $p$-bromophenyl isocyanate $(0.185 \mathrm{~g}$, $0.51 \mathrm{mmol})$ in ether $(10 \mathrm{~mL})$ were stirred for $48 \mathrm{~h}$ at room temperature. After evaporation of the 
solvent, the residue was separated with flash column chromatography (9:1 hexanes/EtOAc) affording $0.167 \mathrm{~g}(59 \%)$ of the title compound: mp $144-145{ }^{\circ} \mathrm{C} ;{ }^{1} \mathbf{H} \mathbf{~ N M R ~}\left(500 \mathrm{MHz}, \mathrm{CDCl}_{3}\right) \delta$ 3.87 (s, 3H), 4.14-4.24 (m, 1H), $4.98(\mathrm{t}, J=11.5 \mathrm{~Hz}, 1 \mathrm{H}), 5.22$ (dd, $J=3.0,12.5 \mathrm{~Hz}, 1 \mathrm{H}), 6.00-$ $6.12(\mathrm{~m}, 2 \mathrm{H}), 6.40(\mathrm{~s}, 1 \mathrm{H}), 6.67(\mathrm{~s}, 1 \mathrm{H}), 6.87(\mathrm{~d}, J=7.0 \mathrm{~Hz}, 2 \mathrm{H}), 6.88-7.60(\mathrm{~m}, 14 \mathrm{H}),{ }^{13} \mathbf{C} \mathbf{~ N M R}$ $\left(125.7 \mathrm{MHz}, \mathrm{CDCl}_{3}\right) \delta 46.7,55.5,60.0,80.7,115.1,121.1,127.6,127.8,127.9,128.2,128.8$, 129.7, 131.4, 131.7, 133.1, 136.1, 137.0, 137.5, 154.9, 169.1. HRMS Calcd for $\mathrm{C}_{29} \mathrm{H}_{26} \mathrm{BrN}_{3} \mathrm{O}_{4}$ : 559.1107; Found: 559.1101. Suitable crystals for X-ray diffraction of the title compound were obtained by recrystallization from 2-propanol/ $\mathrm{CH}_{2} \mathrm{Cl}_{2}$.

\section{References}

1. Suffert, J. J. Org. Chem. 1989, 54, 510.

2. (a) Denmark, S. E.; Juhl, M. Helv. Chim. Acta 2002, submitted. (b) Ranganathan, D.; Rao, C. B.; Ranganathan, S.; Mehrotra, A. K.; Iyengar, R. J. Org. Chem. 1980, 45, 1185. (c) Denmark, S. E.; Marcin, L. J. Org. Chem. 1993, 58, 3850.

3. Gross, K. M.; Jun, Y. M.; Beak, P. J. Org. Chem. 1997, 62, 7679.

4. Mahboobi, S.; Eibler, E.; Koller, M.; KC, S.K.; Popp, A.; Schollmeyer, D. J. Org. Chem. 1999, 64, 4697.

5. Brenner, M.; Seebach, D. Helv. Chim. Acta 1999, 82, 2365.

6. Pollini, G. P.; Barco, A.; De Giuli, G. Synthesis 1972, 42.

7. Meyers, A. I.; Snyder, L. J. Org. Chem. 1993, 58, 36.

8. Weisenburger, G. A.; Beak, P. J. Am. Chem. Soc. 1996, 118, 12218.

9. Curtis, M. D.; Beak, P. J. Org. Chem. 1999, 64, 2996.

10. Amat, M.; Bosch, J.; Hidalgo, J.; Canto, M.; Perez, M.; Llor, N.; Molins, E.; Miravitales, C.; Orozco, M.; Luque, J. J. Org. Chem. 2000, 65, 3074 